\title{
Synthesis of Chiral Thiourea-Thioxanthone Hybrids
}

\author{
Florian Mayr \\ Lisa-Marie Mohr \\ Elsa Rodriguez \\ Thorsten Bach* (i)
}

Department Chemie and Catalysis Research Center (CRC) Technische Universität München, Lichtenbergstr. 4

85747 Garching, Germany

thorsten.bach@ch.tum.de
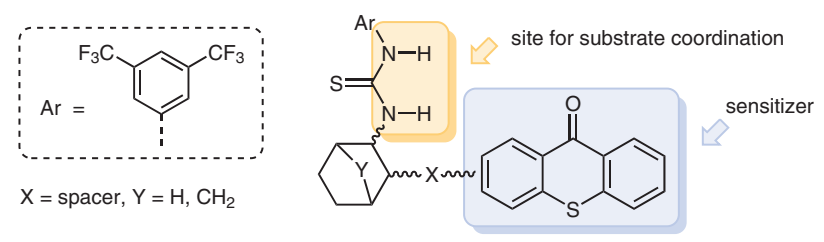

Received: 10.08.2017

Accepted: 12.09.2017

Published online: 19.10 .2017

DOI: 10.1055/s-0036-1590931; Art ID: ss-2017-n0521-op

License terms: cc)

Abstract Four different 1-aminocyclohexanes bearing a tethered thioxanthone group in the 2-position were prepared. The synthesis commenced with the respective $\mathrm{N}$-protected $\beta$-amino acids, the carboxyl group of which was employed for the introduction of the thioxanthone moiety. After construction of the thioxanthone and protecting group removal, the conversion of the amino group into the respective thiourea was accomplished by treatment with $\mathrm{N}$-3,5-bis(trifluorometh$\mathrm{yl}$ )phenyl isothiocyanate and yielded the title compounds in which the thioxanthone resides in different spatial positions relative to the thiourea motif. Overall yields varied between $20-35 \%$.

Key words amino acids, chiral pool, esterification, indium, oxazoles, photochemistry, thiourea, thioxanthone

Thioxanthone (9H-thioxanthen-9-one) exhibits its longest wavelength absorption maximum at $\lambda=376 \mathrm{~nm}(\varepsilon=$ $6200 \mathrm{M}^{-1} \mathrm{~cm}^{-1}$ ) and its triplet energy has been determined as $265 \mathrm{~kJ} \mathrm{~mol}^{-1}$. $^{1}$ The triplet state is populated by direct irradiation with a quantum yield of 0.85 in benzene ${ }^{2}$ and thioxanthone has consequently been employed as a triplet sensitizer for several applications. ${ }^{3}$ Recent interest in visiblelight-induced enantioselective transformations ${ }^{4}$ has led to the development of thioxanthone derivatives in which the chromophore is linked to a chiral backbone. In our group, the linkage was achieved via oxazole annulation to a chiral 1,5,7-trimethyl-3-azabicyclo[3.3.1]nonan-2-one scaffold. The chiral sensitizer was shown to catalyze inter- and intramolecular $[2+2]$ photocycloaddition reactions in a highly enantioselective fashion. ${ }^{5,6}$ The Xiao group developed a bifunctional photocatalyst for an enantioselective aerobic oxidation in which the thioxanthone unit is connected to chiral bisoxazoline complexes by a conformationally flexible ester linkage. $^{7}$

In light of the spectacular success the thiourea binding motif has encountered in organocatalysis,, 8 it seems tempting to employ it also in photochemistry to exert chemo- and stereoselectivity control. The group of Sivaguru showed that a thiourea, which is linked at position C2 to an axially chiral 2 '-hydroxy-1,1'-binaphthyl, is an effective cat- alyst for the enantioselective (86-92\% ee) intramolecular $[2+2]$ photocycloaddition $^{10}$ of 4 -alkenylcoumarins. ${ }^{10,11}$ Three hydrogen bonding interactions were invoked to explain the stereochemical outcome and to account for the bathochromic absorption shift that is responsible for the selective excitation of the catalyst-substrate complex. In additional work, it was discovered that the racemic intermolecular [2+2] photocycloaddition of coumarins can be mediated by achiral thiourea catalysts. ${ }^{12}$ The Beeler group developed a bisthiourea, which is capable of binding a cinnamate at each of its binding sites, and thus increases the regioselectivity of the cinnamate $[2+2]$ photodimerization. ${ }^{13}$

The stoichiometric use of a chiral thiourea was found by our group to induce a notable enantioselectivity $(75 \%$ ee) in the intramolecular thioxanthone-sensitized [2+2] photocycloaddition of a 2,3-dihydropyridone-5-carboxylate. ${ }^{3 g}$ In particular, the last result triggered synthetic efforts aiming at a covalent linkage between a chiral thiourea entity and a thioxanthone and we herein report on the synthesis of such thiourea-thioxanthone hybrids.

The choice of compounds depicted in Figure 1 was inspired by the idea to make a set of chiral 1,2-disubstituted cyclohexanes available in which the thiourea and the thioxanthone unit would be oriented in varying spatial arrangements. Compound 1 bears both units in equatorial positions of a cyclohexane chair while a boat conformation of the cyclohexane is enforced in compound 2 with the thioxanthone and thiourea both located in a pseudoequatorial (exo) position. In compound 3, either thiourea or thioxanthone could be axially positioned with the other substituent being equatorial. Since there was evidence (vide infra) that the thioxanthone was equatorial in $\mathbf{3}$, a fourth cyclohexane derivative $\mathbf{4}$ was designed in which the thioxanthone unit would definitely be axial but the thiourea equatorial. In all catalysts 1-4, the aryl substituent was selected to be the privileged 3,5-bis(trifluoromethyl)phenyl group. ${ }^{14}$

Taking compound $\mathbf{1}$ as an example, a few retrosynthetic considerations are illustrated in Scheme 1. Primary amine $\mathbf{5}$ was chosen as an immediate precursor for the thiourea since it was expected that treatment with 3,5-bis(trifluoro- 

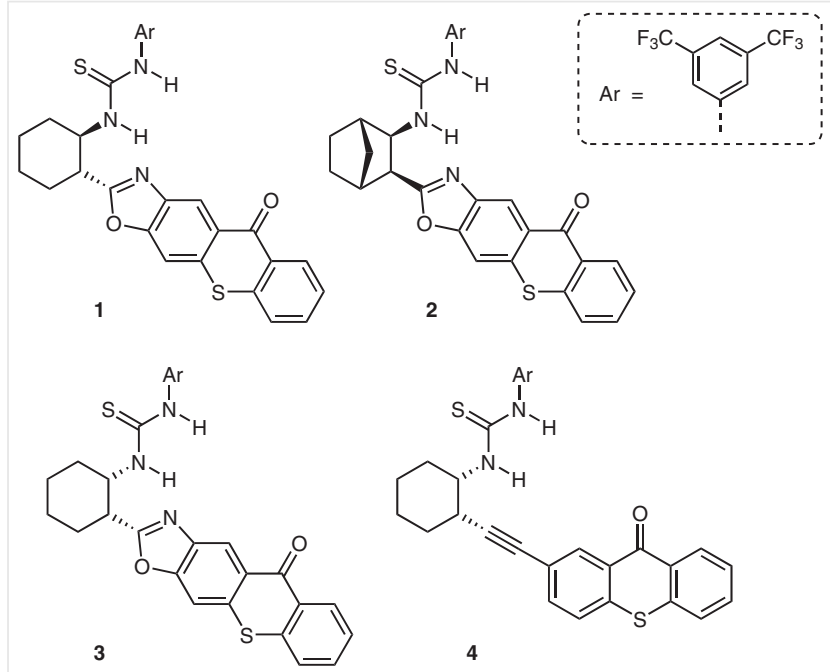

4

Figure 1 Structure of target compounds 1-4 in which a chiral thiourea is linked with a photochemically active thioxanthone via an annulated oxazole or an ethynyl group

methyl)phenyl isothiocyanate ${ }^{15}$ would not interfere with the thioxanthone functionality. In previous work on chiral templates and catalysts for enantioselective reactions, the oxazole group was identified as a reliable linker to be annulated to a given arene. ${ }^{16}$ Formation of the oxazole requires a carboxylic acid precursor and it was expected that the amine group had to be protected prior to the annulation. The arene is normally introduced as its ortho-nitro-substituted aryl ester that upon reduction rearranges to the respective ortho-hydroxy-substituted amide (Scheme 2). For the desired thioxanthone annulation the starting material was thus 3-hydroxy-2-nitrothioxanthone, ${ }^{\text {a }}$ which was to be linked to an appropriately $\mathrm{N}$-protected carboxylic acid derived from enantiopure compound $\mathbf{6}$. Initial attempts to employ a benzyloxycarbonyl or tert-butyloxycarbonyl amine protecting group revealed that they were incompatible with the formation of the aryl ester bond or the subsequent reduction step. Likewise the immediate formation of the thiourea prior to the oxazole annulation was not viable. In most instances, not even the required ester $\mathbf{A}$ (Scheme 2 ) could be formed in useful yields. The phthaloyl protecting group (Phth) was thus selected as a putatively more robust protecting group with compound $\mathbf{7}$ being the desired starting material.

In previous work, the introduction of the thioxanthone had been achieved by reduction of the nitro group with tin(II) chloride in refluxing THF. ${ }^{5 a}$ The cyclization of amide $\mathbf{B}$ to the desired oxazole $\mathbf{C}$ was performed with thionyl chloride/pyridine (py) in refluxing benzene. When attempting to apply these conditions to the synthesis of N-substituted ester derivatives of acid $\mathbf{6}$, there was either no reaction or the formation of decomposition products was observed. The most promising route was the immediate use of the respective thiourea with which a moderate yield of the re- spective ester A was achieved. The reduction could, however, not be performed.

Similar issues were initially encountered with the $\mathrm{N}$ phthaloyl derivative $\mathbf{7}$, which was readily accessible from acid 6 by treatment with phthalic anhydride (8) and triethylamine in benzene (Scheme 3$).{ }^{17}$ Ester formation proceeded smoothly after activation via the acid chloride and reaction with thioxanthone 9..$^{5 a}$ Attempted reduction of compound $\mathbf{1 0}$ with tin(II) chloride or by Pd-catalyzed transfer hydrogenation ${ }^{18}$ remained unsuccessful. Gratifyingly, it

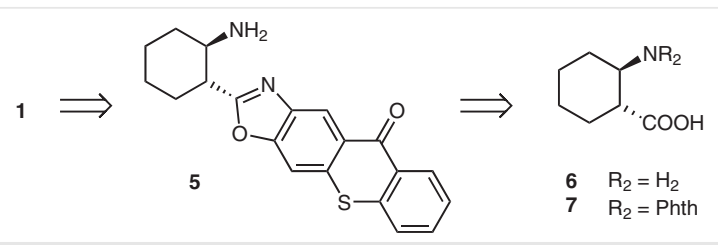

Scheme 1 Retrosynthetic considerations for the construction of thiourea-thioxanthone hybrids $($ Phth $=$ phthaloyl)
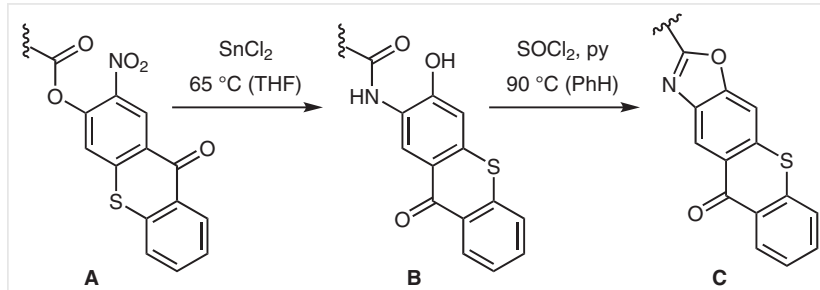

Scheme 2 Synthetic access to oxazole-annulated thioxanthones $C$ starting with (2-nitrothioxanthone-3-yl) ester $\mathbf{A}$

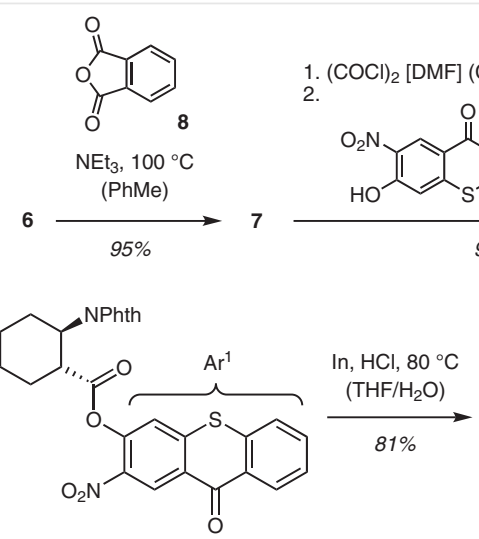

10

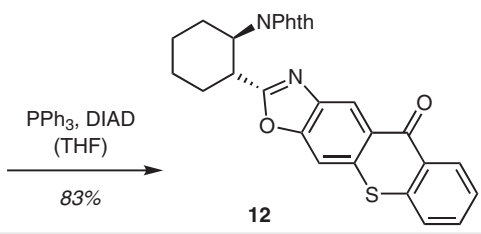

1. $\mathrm{N}_{2} \mathrm{H}_{4}(\mathrm{MeOH})$

2.

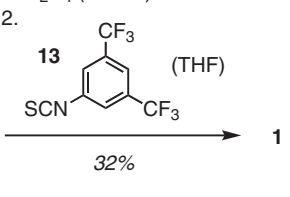

Scheme 3 Synthesis of thiourea-thioxanthone hybrid 1 starting from $\beta$-amino acid 6 
was found that the nitro group could be reduced smoothly with indium in an acidic THF/water mixture. ${ }^{19}$ In the event, the acyl group underwent the expected $\mathrm{O}-\mathrm{N}$ migration and amide $\mathbf{1 1}$ was isolated in $81 \%$ yield. The cyclization to the oxazole ring was achieved under Mitsunobu conditions ${ }^{20}$ while the use of $\mathrm{SOCl}_{2}$ and $\mathrm{POCl}_{3}$ as dehydrating agents failed. Oxazole 12 was obtained in 83\% yield and the removal of the phthaloyl protecting group was accomplished by hydrazinolysis. ${ }^{21}$ Eventually, the desired thiourea was prepared by treatment of amine 5 with 3,5-bis(trifluoromethyl)phenyl isothiocyanate (13). The relatively low overall yield is likely due to material loss in the hydrazinolysis reaction (vide infra).

For the synthesis of compound $\mathbf{2}$, access to enantiopure $\beta$-amino acid $\mathbf{1 4}^{22}$ was required (Scheme 4). This goal was accomplished by applying a known enantiotopos-differentiating methanolysis reaction to the respective succinic anhydride. ${ }^{23}$ Curtius rearrangement, reduction, and ester hydrolysis $\left(100{ }^{\circ} \mathrm{C}\right.$ in 1,4 -dioxane/water) led to the desired compound, which was converted into the phthaloyl derivative 15 by treatment with phthalic anhydride. Hydrogenation of the endocyclic double bond furnished carboxylic acid 16, the enantiomeric excess (ee) of which was determined by chiral HPLC analysis to be $98 \%$. The remaining sequence followed the route developed for compound $\mathbf{1}$. After formation of ester 17, the reduction of the nitro arene with indium and the concomitant rearrangement led to amide 18, which was further transformed under Mitsunobu conditions into thioxanthone 19. In this instance, the attempted removal of the phthaloyl group by hydrazinolysis was not successful but led only to decomposition. Methylamine $^{24}$ led to the cleavage of one imide $\mathrm{N}-\mathrm{C}$ bond but the reaction remained stalled at the stage of the amide. The best result was achieved by treating phthalimide 19 with ethylenediamine (EDA) at $50{ }^{\circ} \mathrm{C} .{ }^{25}$ The protecting group was completely removed and the resulting primary amine was immediately converted into the desired thiourea $\mathbf{2}$.

While the thiourea and the thioxanthone are locked by the rigid norbornane ring in $\mathbf{2}$, a conformationally more flexible cis-substitution at the cyclohexane ring was expected for thiourea 3. The synthesis (Scheme 5) commenced with commercially available $N$-benzoyl (Bz)-protected amino acid 20, which was converted via free acid hydrochloride $\mathbf{2 1}^{26,27}$ into the $N$-phthaloyl-protected amino acid 22. The subsequent sequence of esterification, reduction/rearrangement, and oxazole ring closure proved its reliability and efficiency by providing the respective intermediates 23, 24, and 25 in excellent yields (89-97\%). Treatment with EDA turned out to be also in this case the preferred method for phthaloyl removal and delivered the primary amine for immediate conversion into thiourea 3. The overall yield for the six-step synthesis was $35 \%$.

The conformational preference of compound $\mathbf{3}$ was studied by ${ }^{1} \mathrm{H}$ NMR spectroscopy at ambient temperature.

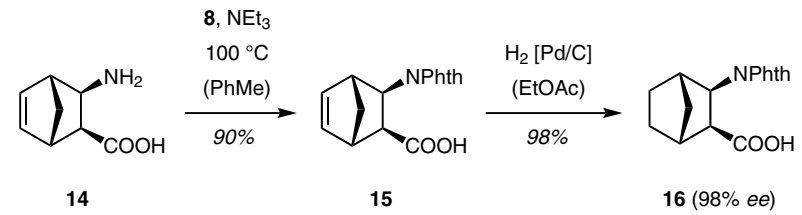

1. $(\mathrm{COCl})_{2}[\mathrm{DMF}]\left(\mathrm{CH}_{2} \mathrm{Cl}_{2}\right)$

2. $9, \mathrm{NEt}_{3}[\mathrm{DMAP}] 0^{\circ} \mathrm{C} \rightarrow \mathrm{rt}$ $\left(\mathrm{CH}_{2} \mathrm{Cl}_{2}\right)$

$99 \%$

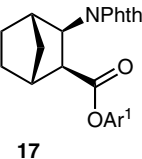

$\begin{gathered}\text { In, } \mathrm{HCl}, 80^{\circ} \mathrm{C} \\ \left(\mathrm{THF} / \mathrm{H}_{2} \mathrm{O}\right)\end{gathered}$
$\underset{87 \%}{\longrightarrow}$

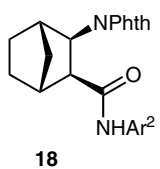

18

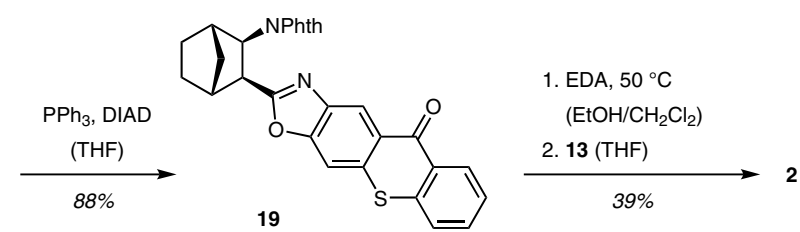

Scheme 4 Synthesis of thiourea-thioxanthone hybrid $\mathbf{2}$ starting from $\beta$-amino acid 14
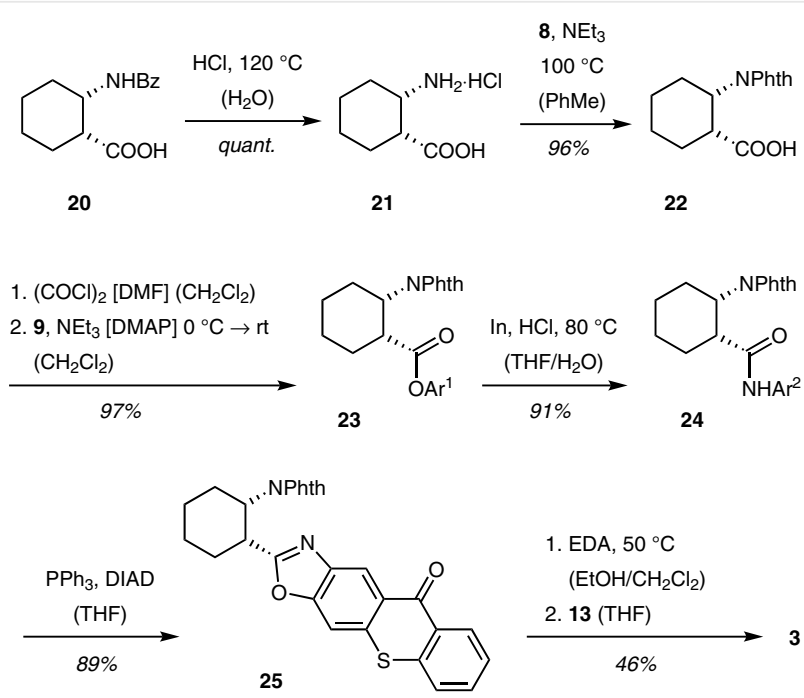

1. EDA, $50^{\circ} \mathrm{C}$ $\left(\mathrm{EtOH} / \mathrm{CH}_{2} \mathrm{Cl}_{2}\right.$ ) 2. 13 (THF)

$46 \%$ 3

Scheme 5 Synthesis of thiourea-thioxanthone hybrid $\mathbf{3}$ starting from $\mathrm{N}$-benzoyl-protected $\beta$-amino acid 20

The proton at carbon atom $\mathrm{C} 1$ (Scheme 6 ) is expected to exhibit in conformation $\mathbf{3}^{\prime}$ one large coupling constant due to the axial-axial coupling $\left({ }^{3} J_{a a}\right)$ and two small coupling constants due the axial-equatorial coupling $\left({ }^{3} J_{a e}\right) .{ }^{28}$ Indeed, its precursor 25 showed exactly this pattern with ${ }^{3} J_{a a}=13.2 \mathrm{~Hz}$ and ${ }^{3} J_{a e}=5.2 \mathrm{~Hz}$ and ${ }^{3} J_{a e}=3.6 \mathrm{~Hz}$. The proton at $\mathrm{C} 2$ appeared as a virtual quartet with an average coupling constant of ${ }^{3} \mathrm{~J}$ $\cong 5.2 \mathrm{~Hz}$. Related ${ }^{1} \mathrm{H}$ NMR data were recorded for compounds $\mathbf{2 3}$ and 24. However, the situation was different for compound 3. The ${ }^{1} \mathrm{H}$ NMR spectrum revealed for $\mathrm{H} 1 \mathrm{a}$ signal that appeared as virtual tt with two coupling constants of ${ }^{3} \mathrm{~J}$ 
$\cong 8.3 \mathrm{~Hz}$ and ${ }^{3} \mathrm{~J} \cong 4.0 \mathrm{~Hz}$. While one of the larger signal splittings is due to the coupling to $\mathrm{NH}$, the remaining coupling pattern $\left({ }^{3} \mathrm{~J}=8.3,4.0,4.0 \mathrm{~Hz}\right)$ results from coupling to vicinal protons. Likewise, the proton $\mathrm{H} 2$ changed its signal pattern and appeared as a virtual dt with ${ }^{3} J=7.4 \mathrm{~Hz}$ and ${ }^{3} J \cong 4.3 \mathrm{~Hz}$.
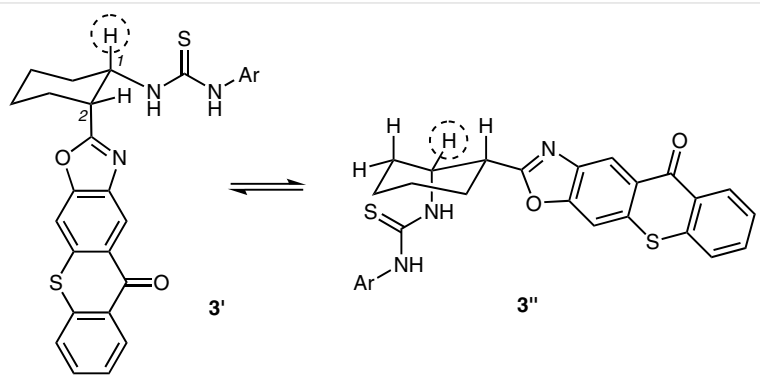

Scheme 6 Equilibrium between conformations 3' and 3" of compound $\mathbf{3}$ as suggested by the ${ }^{1} \mathrm{H}$ NMR data for the indicated proton (see narrative)

Apparently there is no clear preference for either conformer 3' or 3" and neither proton $\mathrm{H} 1$ nor $\mathrm{H} 2$ has a clear preference for the axial position. Rather it seems as if an equilibrium was established at ambient temperature most likely due to the fact that the two substituents at C1 and C2 are similar in size. In order to establish a less ambiguous conformational situation within the cis-substituted cyclohexane ring, a thiourea-thioxanthone hybrid $\mathbf{4}$ was devised in which the thioxanthone was linked to the cyclohexane ring by the linear, sterically unencumbered ${ }^{29}$ ethynyl group. The synthesis of this compound started from hydrochloride 21, which was reduced with $\mathrm{LiAlH}_{4}$ in THF to furnish amino alcohol 26 in 93\% yield (Scheme 7). Upon tert-butoxycarbonyl (Boc) protection of the amino group, alcohol $27^{30}$ was subjected to a Swern oxidation. ${ }^{31}$ The resulting aldehyde $\mathbf{2 8}$ was converted into the terminal alkyne 29 by Seyferth-Gilbert homologization. ${ }^{32}$ Sonogashira cross-coupling ${ }^{33}$ with the known 2-bromothioxanthone $(\mathbf{3 0})^{34}$ gave the 2-substituted thioxanthone $\mathbf{3 1}$, which could be readily deprotected with trifluoroacetic acid (TFA) to give the desired amine $\mathbf{3 2}$. As in the previous syntheses, the desired thiourea was generated by treatment of the amine with 3,5-bis(trifluoromethyl)phenyl isothiocyanate (13).

As expected, compound $\mathbf{4}$ displays preferred conformation $\mathbf{4}^{\prime}$ in which the tethered thioxanthone is axially positioned. The ${ }^{1} \mathrm{H}$ NMR coupling pattern of proton $\mathrm{H} 1$ is a virtual ddt with coupling constants of ${ }^{3} \mathrm{~J}=12.4 \mathrm{~Hz},{ }^{3} \mathrm{~J}=8.5 \mathrm{~Hz}$, and ${ }^{3} J \cong 3.9 \mathrm{~Hz}$. Since the coupling constant of ${ }^{3} J=8.5 \mathrm{~Hz}$ could be clearly assigned to the vicinal $\mathrm{CH}-\mathrm{NH}$ coupling the other coupling constants are due to vicinal $\mathrm{CH}-\mathrm{CH}$ coupling with ${ }^{3} J_{a a}=12.4 \mathrm{~Hz}$ and ${ }^{3} J_{a e} \cong 3.9 \mathrm{~Hz}$. Likewise, the equatorial proton $\mathrm{H} 2$ shows a virtual quartet with the ${ }^{3} J_{a e} \cong{ }^{3} J_{e e}=3.6$ $\mathrm{Hz}$.

The UV/Vis spectra of the new thioxanthones are all similar (see the Supporting Information) and the spectrum

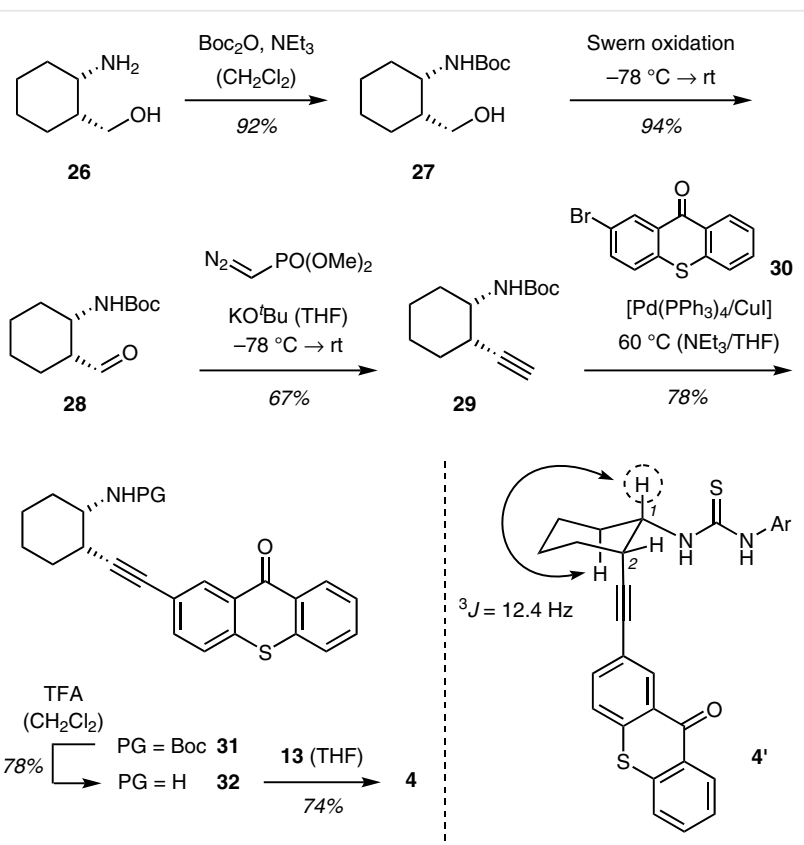

Scheme 7 Preferred conformation $\mathbf{4}^{\prime}$ and synthesis of thiourea-thioxanthone hybrid $\mathbf{4}$ starting from $\beta$-amino alcohol 26

of compound $\mathbf{4}$ is representatively shown in Figure 2. The long-wavelength absorption between 370 and $420 \mathrm{~nm}$ with a $\lambda_{\max }=392 \mathrm{~nm}\left(\varepsilon=3340 \mathrm{M}^{-1} \mathrm{~cm}^{-1}\right)$ is likely due to the thioxanthone chromophore while the strong absorptions setting in below $320 \mathrm{~nm}$ are attributed to allowed transitions of the thiourea and the thioxanthone. In line with their UV/Vis spectra, the compounds are yellow-colored solids. Phosphorescence data have not yet been obtained but it was expected that the compounds will act as triplet sensitizers in the same fashion as does the parent thioxanthone.

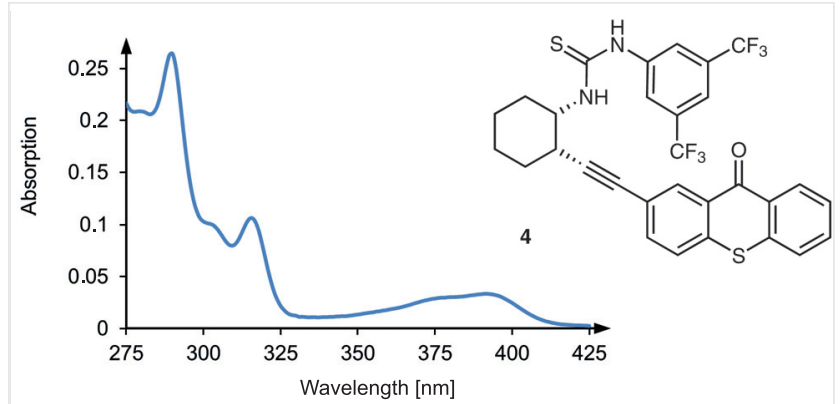

Figure $2 \mathrm{UV} / \mathrm{Vis}$ spectrum of thiourea-thioxanthone hybrid 4 in $\mathrm{CH}_{2} \mathrm{Cl}_{2}$ solution $(c=0.1 \mathrm{mM})$

In preliminary and non-optimized experiments, it was probed whether the thiourea-thioxanthone hybrids would act as catalysts of visible-light-induced reactions. Along these lines, catalyst $\mathbf{4}$ was employed in the photocyclization of 2-aryloxycyclohex-2-enones, ${ }^{35}$ which has recently 
received increasing attention. ${ }^{3 \mathrm{~h}, 36}$ Gratifyingly, we found that the reaction of compound 33, which does not proceed at $\lambda=$ $419 \mathrm{~nm}$ in the absence of a sensitizer could be successfully promoted by catalyst 4 (Scheme 8 ). Although the yield and enantioselectivity of product $\mathbf{3 4}$ was low, the experiment demonstrates that the thiourea-thioxanthone hybrids are catalytically active and that an asymmetric binding event at the NH-hydrogen atoms of the thiourea is likely to occur.

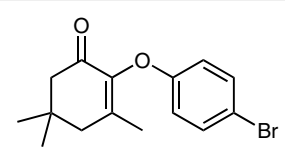

33

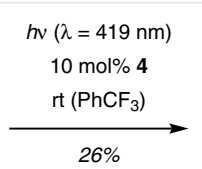

(49\% conv.)

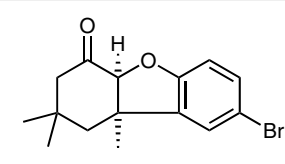

$34(12 \%$ ee $)$
Scheme 8 Sensitized photocyclization of 2-(4-bromophenoxy)-3,5,5trimethylcyclohex-2-enone (33)

In summary, we have successfully synthesized four thiourea-thioxanthone hybrid compounds from the respective $\beta$-amino acids. For the attachment of the thioxanthone by oxazole annulation we have devised a generally applicable and mild reaction sequence. The compounds exhibit a two-point hydrogen bonding site at the thiourea and it is expected that the thioxanthone will act as a sensitizer to promote photochemical reactions of bound substrates in the respective 1:1 complexes. Work along these lines is currently underway in our laboratories and will be reported in due course.

All reactions involving moisture-sensitive chemicals were carried out in flame-dried glassware under positive pressure of argon with magnetic stirring. THF, $\mathrm{CH}_{2} \mathrm{Cl}_{2}$, and $\mathrm{Et}_{2} \mathrm{O}$ were purified using a SPS-800 solvent purification system (M. Braun). TLC was performed on silicacoated glass plates (silica gel $\left.60 \mathrm{~F}^{254}\right)$ with detection by UV $(\lambda=254$ $\mathrm{nm})$, cerium ammonium molybdate (CAM) or $\mathrm{KMnO}_{4}\left(0.5 \%\right.$ in $\left.\mathrm{H}_{2} \mathrm{O}\right)$ with subsequent heating. Flash chromatography was performed on silica gel 60 (Merck, 230-400 mesh) with the indicated eluent. Common solvents for chromatography [pentane (Pn), cyclohexane (Chx), EtOAc, $\mathrm{CH}_{2} \mathrm{Cl}_{2}, \mathrm{Et}_{2} \mathrm{O}, \mathrm{MeOH}$ ] were distilled prior to use. Solutions refer to sat. aq solutions, unless otherwise stated. IR spectra were recorded on a JASCO IR-4100 (ATR). MS/HRMS measurements were performed on a Finnigan MAT 8200 or Thermo Fisher DFS (EI)/Finnigan LSQ classic or Thermo Fisher LTY Orbitrap XL (ESI). ${ }^{1} \mathrm{H}$ and ${ }^{13} \mathrm{C}$ NMR spectra were recorded in $\mathrm{CDCl}_{3}$ or DMSO- $d_{6}$ at $300 \mathrm{~K}$ on a Bruker AV-360, Bruker AVHD-400, Bruker AV-500, or a Bruker AVHD-500 instrument. Chemical shifts are reported relative to $\mathrm{CHCl}_{3}(\delta=7.26)$ or DMSO- $d_{5}$ $(\delta=2.50)$. Apparent multiplets that occur as a result of the accidental equality of coupling constants to those of magnetically non-equivalent protons are marked as virtual (virt.). The multiplicities of the ${ }^{13} \mathrm{C}$ NMR signals were determined by DEPT-edited phase sensitive HSQC experiments. Assignments are based on COSY, HMBC, HSQC, and NOESY experiments. Signals that could not be assigned unambiguously are marked with an asterisk $\left({ }^{*}\right)$. UV/Vis Spectroscopy was performed on a PerkinElmer Lambda 35 UV/Vis spektometer. Unless otherwise mentioned, UV spectra were recorded using a Hellma precision cell made of quartz with a pathway of $1 \mathrm{~mm}$. Solvents and concentrations are given for each spectrum. Rotation value measure- ments were performed on a Bellingham + Stanley ADP400+ with a $0.05 \mathrm{dm}$ cuvette at $\lambda=589 \mathrm{~nm}$ (Na D-line) at room temperature. The specific rotation is given in $10^{-1}$ grad cm $\mathrm{g}^{-1}$, the concentration is given in $\mathrm{g} / 100 \mathrm{~mL}$. Analytical HPLC was performed using a chiral stationary phase (flow rate: $1.0 \mathrm{~mL} / \mathrm{min}$, column type and eluent are given for the corresponding compounds) and UV detection ( $\lambda=210 \mathrm{~nm}$ or $254 \mathrm{~nm})$ at $20^{\circ} \mathrm{C}$.

\section{$(1 R, 2 R)-2-(1 ', 3 '-$ Dioxoisoindolin-2'-yl)cyclohexane-1-carboxylic Acid (7)}

Amino acid 6 (200 mg, $1.40 \mathrm{mmol}, 1.00$ equiv) was dissolved in anhyd toluene $(20 \mathrm{~mL})$. Subsequently, phthalic anhydride $(218 \mathrm{mg}$, $1.47 \mathrm{mmol}, 1.05$ equiv) and $\mathrm{NEt}_{3}(283 \mathrm{mg}, 0.38 \mathrm{~mL}, 2.80 \mathrm{mmol}$, 2.00 equiv) were added. The mixture was stirred at $100^{\circ} \mathrm{C}$. After $18 \mathrm{~h}$, the solution was cooled to $\mathrm{rt}$ and washed with aq $3 \mathrm{M} \mathrm{HCl}(100 \mathrm{~mL})$. The aqueous layer was extracted with $\mathrm{CH}_{2} \mathrm{Cl}_{2}(3 \times 100 \mathrm{~mL})$. The combined organic layers were dried $\left(\mathrm{Na}_{2} \mathrm{SO}_{4}\right)$, filtered and the solvent was removed under reduced pressure. The desired product was obtained as a colorless solid (363 mg, $1.33 \mathrm{mmol}, 95 \%$ ) and was used in the next step without purification; $\mathrm{mp} 138-140{ }^{\circ} \mathrm{C} ;[\alpha]_{\mathrm{D}}{ }^{20}-10(c=1.00$, $\mathrm{CH}_{2} \mathrm{Cl}_{2}$ ).

IR (ATR): $3514(\mathrm{~m}, \mathrm{OH}), 3369(\mathrm{w}, \mathrm{OH}), 2936\left(\mathrm{~m}, \mathrm{CH}_{\mathrm{sp} 3}\right), 2861(\mathrm{~m}$, $\left.\mathrm{CH}_{\mathrm{sp} 3}\right), 1740(\mathrm{~m}), 1697$ (s, C=0), $1376(\mathrm{~m}), 1330(\mathrm{~m}), 1204(\mathrm{w}), 1077$ $(\mathrm{m}), 718 \mathrm{~cm}^{-1}(\mathrm{~m})$.

${ }^{1} \mathrm{H} \mathrm{NMR}\left(400 \mathrm{MHz}, \mathrm{CDCl}_{3}\right): \delta=1.28-1.60$ (m, $\left.3 \mathrm{H}, \mathrm{H}-5, \mathrm{H}-6\right), 1.69-1.90$ (m, $3 \mathrm{H}, \mathrm{H}-3, \mathrm{H}-4), 1.99-2.20$ (m, $2 \mathrm{H}, \mathrm{H}-3, \mathrm{H}-6), 3.43$ (virt. td, ${ }^{3} \cong$ ${ }^{3} \mathrm{~J}=12.1 \mathrm{~Hz},{ }^{3} \mathrm{~J}=3.7 \mathrm{~Hz}, 1 \mathrm{H}, \mathrm{H}-1$ ), 4.28 (ddd, ${ }^{3} \mathrm{~J}=12.1,11.3,4.0 \mathrm{~Hz}, 1$ $\mathrm{H}, \mathrm{H}-2$ ), 7.67 (dd, ${ }^{3} \mathrm{~J}=5.5 \mathrm{~Hz},{ }^{4} \mathrm{~J}=3.0 \mathrm{~Hz}, 2 \mathrm{H}, \mathrm{H}-5^{\prime}, \mathrm{H}-6^{\prime}$ ), 7.78 (dd, $\left.{ }^{3} \mathrm{~J}=5.5 \mathrm{~Hz},{ }^{4} \mathrm{~J}=3.0 \mathrm{~Hz}, 2 \mathrm{H}, \mathrm{H}-4^{\prime}, \mathrm{H}-7^{\prime}\right), 10.40$ (br s, $1 \mathrm{H}, \mathrm{OH}$ ).

${ }^{13} \mathrm{C}$ NMR (101 MHz, $\left.\mathrm{CDCl}_{3}\right): \delta=24.7(\mathrm{t}, \mathrm{C}-5), 25.3(\mathrm{t}, \mathrm{C}-4), 29.7(\mathrm{t}, \mathrm{C}-6)$, 29.8 (t, C-3), 44.7 (d, C-1), 51.0 (d, C-2), 123.4 (d, 2 C, C-4', C-7'), 131.9 (s, 2 C, C-3a', C-7a'), 134.0 (d, 2 C, C-5', C-6'), 168.2 (s, 2 C, C-1', C-3'), $179.2(\mathrm{~s}, \mathrm{COOH})$.

MS (EI, $70 \mathrm{eV}): m / z(\%)=273\left(8,[\mathrm{M}]^{+}\right), 227\left(12,\left[\mathrm{M}-\mathrm{CH}_{3}\right]^{+}\right), 186(15)$, 160 (63), $148\left(20,\left[\mathrm{C}_{8} \mathrm{H}_{6} \mathrm{NO}_{2}\right]^{+}\right), 91\left(100,\left[\mathrm{C}_{7} \mathrm{H}_{7}\right]^{+}\right)$.

HRMS (EI): $m / z$ [M] $]^{+}$calcd for $\mathrm{C}_{15} \mathrm{H}_{15} \mathrm{NO}_{4}$ : 273.1001; found: 273.0996 .

\section{2'-Nitro-9'-oxo-9'H-thioxanthen-3'-yl (1R,2R)-2-(1",3"-Dioxoiso- indolin-2"-yl)cyclohexane-1-carboxylate (10)}

The protected amino acid 7 (666 mg, $2.34 \mathrm{mmol}, 1.10$ equiv) was dissolved in anhyd $\mathrm{CH}_{2} \mathrm{Cl}_{2}(33 \mathrm{~mL})$. Oxalyl chloride $(297 \mathrm{mg}, 0.20 \mathrm{~mL}$, $2.34 \mathrm{mmol}, 1.10$ equiv) and a catalytic amount of DMF (7 drops) was added at rt. The mixture was stirred at $\mathrm{rt}$ for $3 \mathrm{~h}$. In parallel, thioxanthone $\mathbf{9}$ (581 mg, $2.13 \mathrm{mmol}, 1.00$ equiv) and a catalytic amount of 4dimethylaminopyridine (10 crystals) was dissolved in anhyd $\mathrm{CH}_{2} \mathrm{Cl}_{2}$ $(33 \mathrm{~mL})$ and cooled to $0{ }^{\circ} \mathrm{C}$. At this temperature, $\mathrm{NEt}_{3}(647 \mathrm{mg}$, $0.89 \mathrm{~mL}, 6.39 \mathrm{mmol}, 3.00$ equiv) was added. Subsequently, the previously prepared solution of acid chloride was added slowly. The mixture was warmed to $\mathrm{rt}$ and stirred overnight. After $18 \mathrm{~h}$, aq $\mathrm{NH}_{4} \mathrm{Cl}$ $(100 \mathrm{~mL})$ was added and the layers were separated. The aqueous layer was extracted with $\mathrm{CH}_{2} \mathrm{Cl}_{2}(3 \times 150 \mathrm{~mL})$ and the combined organic layers were washed with aq $2 \mathrm{M} \mathrm{NaOH}(200 \mathrm{~mL})$, dried $\left(\mathrm{Na}_{2} \mathrm{SO}_{4}\right)$, and filtered. The solvent was evaporated and the desired product was obtained without further purification as a yellowish solid $(1.11 \mathrm{~g}$, $2.11 \mathrm{mmol}, 99 \%) ; \mathrm{mp} 202-205{ }^{\circ} \mathrm{C} ; R_{f}=0.89\left(\mathrm{CH}_{2} \mathrm{Cl}_{2} / \mathrm{MeOH} 98: 2\right)$ [UV, $\left.\mathrm{KMnO}_{4}\right] ;[\alpha]_{\mathrm{D}}{ }^{20}-92\left(c=1.00, \mathrm{CH}_{2} \mathrm{Cl}_{2}\right)$.

IR (ATR): $3086\left(\mathrm{w}, \mathrm{CH}_{\mathrm{sp} 2}\right), 3036\left(\mathrm{w}, \mathrm{CH}_{\mathrm{sp} 2}\right), 2933\left(\mathrm{~m}, \mathrm{CH}_{\mathrm{sp} 3}\right), 2864(\mathrm{~m}$, $\left.\mathrm{CH}_{\mathrm{sp} 3}\right), 1766(\mathrm{~s}, \mathrm{C}=\mathrm{O}), 1708(\mathrm{~s}, \mathrm{C}=\mathrm{O}), 1635(\mathrm{~s}), 1605(\mathrm{~s}), 1519(\mathrm{~m}$, $\left.\mathrm{C}=\mathrm{C}_{\mathrm{sp} 2}\right), 1379(\mathrm{~m}), 1341(\mathrm{~s}), 1293(\mathrm{~m}), 1108(\mathrm{~m}), 1077(\mathrm{~m}), 1025(\mathrm{~m})$, $717 \mathrm{~cm}^{-1}(\mathrm{~m})$. 
${ }^{1} \mathrm{H} \mathrm{NMR}\left(400 \mathrm{MHz}, \mathrm{CDCl}_{3}\right): \delta=1.50-1.56(\mathrm{~m}, 2 \mathrm{H}, \mathrm{H}-4, \mathrm{H}-5), 1.78$ (virt. $\left.\mathrm{td},{ }^{3} \mathrm{~J} \cong{ }^{3} \mathrm{~J}=12.8 \mathrm{~Hz},{ }^{3} \mathrm{~J}=3.4 \mathrm{~Hz}, 1 \mathrm{H}, \mathrm{H}-4\right), 1.90-1.99$ (m, $3 \mathrm{H}, \mathrm{H}-3, \mathrm{H}-5$, H-6), 2.30 (virt. qd, ${ }^{3} \cong \cong 3 \mathrm{~J}=12.4 \mathrm{~Hz},{ }^{3} \mathrm{~J}=2.8 \mathrm{~Hz}, 1 \mathrm{H}, \mathrm{H}-6$ ), 2.49 (virt. $\left.\mathrm{td},{ }^{3} \mathrm{~J} \cong 3^{3}=13.7 \mathrm{~Hz},{ }^{3} \mathrm{~J}=1.8 \mathrm{~Hz}, 1 \mathrm{H}, \mathrm{H}-3\right), 3.84\left(\mathrm{ddd},{ }^{3} \mathrm{~J}=12.3 \mathrm{~Hz}\right.$, $11.3 \mathrm{~Hz}, 3.7 \mathrm{~Hz}, 1 \mathrm{H}, \mathrm{H}-1$ ), 4.55 (ddd, ${ }^{3} \mathrm{~J}=12.3 \mathrm{~Hz}, 11.3 \mathrm{~Hz}, 4.0 \mathrm{~Hz}, 1 \mathrm{H}$, $\mathrm{H}-2), 7.17$ (s, $\left.1 \mathrm{H}, \mathrm{H}-4^{\prime}\right), 7.54-7.58$ (m, $\left.2 \mathrm{H}, \mathrm{H}-5^{\prime}, \mathrm{H}-7^{\prime}\right), 7.69$ (ddd, $\left.{ }^{3} J=8.3,7.0 \mathrm{~Hz},{ }^{4} J=1.6 \mathrm{~Hz}, 1 \mathrm{H}, \mathrm{H}-6^{\prime}\right), 7.74\left(\mathrm{dd},{ }^{3} J=5.5 \mathrm{~Hz},{ }^{4} \mathrm{~J}=3.1 \mathrm{~Hz}\right.$, $\left.2 \mathrm{H}, \mathrm{H}-5^{\prime \prime}, \mathrm{H}-6^{\prime \prime}\right), 7.87$ (dd, $\left.{ }^{3} \mathrm{~J}=5.5 \mathrm{~Hz},{ }^{4} \mathrm{~J}=3.1 \mathrm{~Hz}, 2 \mathrm{H}, \mathrm{H}-4^{\prime \prime}, \mathrm{H}-7^{\prime \prime}\right)$, 8.54-8.60 (m, 1 H, H-8'), 9.20 (s, $\left.1 \mathrm{H}, \mathrm{H}-1^{\prime}\right)$.

${ }^{13} \mathrm{C}$ NMR $\left(100 \mathrm{MHz}, \mathrm{CDCl}_{3}\right): \delta=24.6(\mathrm{t}, \mathrm{C}-4), 25.4(\mathrm{t}, \mathrm{C}-5), 29.5(\mathrm{t}, \mathrm{C}-6)$, 29.6 (t, C-3), 45.4 (d, C-1), 51.1 (d, C-2), 122.0 (d, C-4'), 123.4 (d, 2 C, C-4", C-7"), 126.3 (d, C-5'), 127.1 (s, C-9a'), 127.8 (d, C-7'), 128.3 (d, C1'), 128.5 (s, C-8a'), 130.3 (d, C-8'), 132.0 (s, 2 C, C-3a", 7a"), 133.4 (d, C-6'), 134.2 (d, 2 C, C-5", C-6"), 135.7 (s, C-4b'), 140.2 (s, C-3'), 144.1 (s, C-4a'), 145.9 (s, C-2'), 168.3 (s, 2 C, C-1", C-3"), 170.9 (s, COO), 177.9 (s, C-9').

MS (EI, $70 \mathrm{eV}): m / z(\%)=528\left(3,[\mathrm{M}]^{+}\right), 273\left(29,\left[\mathrm{C}_{13} \mathrm{H}_{7} \mathrm{NO}_{4} \mathrm{~S}\right]^{+}\right), 256$ $\left(100,\left[\mathrm{C}_{15} \mathrm{H}_{14} \mathrm{NO}_{3}\right]^{+}\right), 148\left(79,\left[\mathrm{C}_{8} \mathrm{H}_{6} \mathrm{NO}_{2}\right]^{+}\right)$.

HRMS (EI): $m / z$ [M] calcd for $\mathrm{C}_{28} \mathrm{H}_{20} \mathrm{~N}_{2} \mathrm{O}_{7} \mathrm{~S}$ : 528.0991; found: 528.0995 .

\section{(1R,2R)-2-(1",3"-Dioxoisoindolin-2"-yl)-N-(3'-hydroxy-9'-oxo-9'H- thioxanthen-2'-yl)cyclohexane-1-carboxamide (11)}

Ester 10 (406 mg, $768 \mu \mathrm{mol}, 1.00$ equiv) was dissolved in THF (27 mL) and $\mathrm{H}_{2} \mathrm{O}(27 \mathrm{~mL})$ was added. To this solution $\mathrm{In}(441 \mathrm{mg}, 3.84 \mathrm{mmol}$, 5.00 equiv) and concd $\mathrm{HCl}(0.75 \mathrm{~mL})$ were added. The mixture was stirred at $80^{\circ} \mathrm{C}$ for $18 \mathrm{~h}$. After this time, the solution was cooled to rt and $\mathrm{CH}_{2} \mathrm{Cl}_{2}(50 \mathrm{~mL})$ was added. The mixture was filtered over Celite and washed with aq $\mathrm{NaHCO}_{3}(100 \mathrm{~mL})$. The aqueous layer was extracted with $\mathrm{CH}_{2} \mathrm{Cl}_{2}(3 \times 100 \mathrm{~mL})$. The combined organic layers were dried $\left(\mathrm{Na}_{2} \mathrm{SO}_{4}\right)$, filtered, and the solvent was removed under reduced pressure. Purification by flash chromatography $\left(4 \times 20 \mathrm{~cm}, \mathrm{CH}_{2} \mathrm{Cl}_{2}\right.$ / $\mathrm{MeOH}$ 95:5) furnished the title compound as an orange-colored solid (310 mg, $622 \mu \mathrm{mol}, 81 \%) ; \mathrm{mp} 150-154{ }^{\circ} \mathrm{C} ; R_{f}=0.51\left(\mathrm{CH}_{2} \mathrm{Cl}_{2} / \mathrm{MeOH}\right.$ 95:5) [UV, $\left.\mathrm{KMnO}_{4}\right] ;[\alpha]_{\mathrm{D}}^{20}+46\left(c=1.00, \mathrm{CH}_{2} \mathrm{Cl}_{2}\right)$.

IR (ATR): 3296 (w, NH), $3066(\mathrm{w}, \mathrm{OH}), 2931\left(\mathrm{~m}, \mathrm{CH}_{\mathrm{sp} 3}\right), 2857(\mathrm{~m}$, $\left.\mathrm{CH}_{\mathrm{sp} 3}\right), 1766(\mathrm{w}), 1700(\mathrm{~s}, \mathrm{C}=\mathrm{O}), 1588\left(\mathrm{~m}, \mathrm{C}=\mathrm{C}_{\mathrm{sp} 2}\right), 1517\left(\mathrm{~m}, \mathrm{C}=\mathrm{C}_{\mathrm{sp} 2}\right)$, $1438\left(\mathrm{~m}, \mathrm{CH}_{\mathrm{sp} 3}\right), 1369(\mathrm{~m}), 1294(\mathrm{w}), 1262(\mathrm{w}), 1077(\mathrm{~m}), 717 \mathrm{~cm}^{-1}$ $\left(\mathrm{m}, \mathrm{CH}_{\mathrm{sp} 2}\right)$.

${ }^{1} \mathrm{H} \mathrm{NMR}\left(400 \mathrm{MHz}, \mathrm{CDCl}_{3}\right): \delta=1.00-1.17(\mathrm{~m}, 1 \mathrm{H}, \mathrm{H}-5), 1.42-1.54(\mathrm{~m}$, $1 \mathrm{H}, \mathrm{H}-4), 1.63-1.82$ (m, $4 \mathrm{H}, \mathrm{H}-3, \mathrm{H}-4, \mathrm{H}-5, \mathrm{H}-6), 2.00$ (virt. dq, $\left.{ }^{3} J=12.7 \mathrm{~Hz},{ }^{3} \cong \cong 3 \mathrm{~J}=3.6 \mathrm{~Hz}, 1 \mathrm{H}, \mathrm{H}-3\right), 2.06-2.18(\mathrm{~m}, 1 \mathrm{H}, \mathrm{H}-6), 3.82$ (virt. td, ${ }^{3} \mathrm{~J} \cong{ }^{3} \mathrm{~J}=11.8 \mathrm{~Hz},{ }^{3} \mathrm{~J}=3.7 \mathrm{~Hz}, 1 \mathrm{H}, \mathrm{H}-1$ ), 4.53 (virt. td, ${ }^{3} \mathrm{~J} \cong$ $\left.{ }^{3} \mathrm{~J}=11.8 \mathrm{~Hz},{ }^{3} \mathrm{~J}=4.0 \mathrm{~Hz}, 1 \mathrm{H}, \mathrm{H}-2\right), 6.99\left(\mathrm{~s}, 1 \mathrm{H}, \mathrm{H}-4^{\prime}\right), 7.44-7.48(\mathrm{~m}, 2$ $\left.\mathrm{H}, \mathrm{H}-7^{\prime}, \mathrm{H}-5^{\prime}\right), 7.53-7.56$ ( $\left.\mathrm{m}, 1 \mathrm{H}, \mathrm{H}-6^{\prime}\right), 7.59$ (dd, ${ }^{3} \mathrm{~J}=5.5 \mathrm{~Hz}$, $\left.{ }^{4} J=3.0 \mathrm{~Hz}, 2 \mathrm{H}, \mathrm{H}-5^{\prime \prime}, \mathrm{H}-6^{\prime \prime}\right), 7.73$ (dd, ${ }^{3} \mathrm{~J}=5.5 \mathrm{~Hz},{ }^{4} \mathrm{~J}=3.0 \mathrm{~Hz}, 2 \mathrm{H}, \mathrm{H}-4^{\prime \prime}$, H-7"), 8.47 (s, $\left.1 \mathrm{H}, \mathrm{H}-1^{\prime}\right), 8.61$ (dd, ${ }^{3} J=8.4 \mathrm{~Hz},{ }^{4} \mathrm{~J}=1.6 \mathrm{~Hz}, 1 \mathrm{H}, \mathrm{H}-8^{\prime}$ ), $9.33(\mathrm{~s}, 1 \mathrm{H}, \mathrm{NH}), 10.81(\mathrm{~s}, 1 \mathrm{H}, \mathrm{OH})$.

${ }^{13} \mathrm{C} \mathrm{NMR}\left(101 \mathrm{MHz}, \mathrm{CDCl}_{3}\right): \delta=24.7(\mathrm{t}, \mathrm{C}-5), 25.2(\mathrm{t}, \mathrm{C}-4), 29.8(\mathrm{t}, \mathrm{C}-3)$, 30.9 (t, C-6), 47.4 (d, C-1), 51.8 (d, C-2), 114.6 (d, C-4'), 121.9 (s, C-4a'), 123.4 (d, 2 C, C-4", C-7"), 123.7 (d, C-1'), 126.0 (d, C-7'), 126.4 (d, C5'), 126.9 (s, C-3'), 128.6 (s, C-8a'), 130.0 (d, C-8'), 131.8 (s, 2 C, C-3a", C-7a"), 132.2 (d, C-6'), 134.1 (d, 2 C, C-5", C-6"), 136.6 (s, C-9a'), 137.5 (s, C-4b'), 153.8 (s, C-2'), 168.5 (s, 2 C, C-1", C-3"), 175.2 (s, CON), $179.3\left(\mathrm{~s}, \mathrm{C}-9^{\prime}\right)$.

MS (EI, $70 \mathrm{eV}): m / z(\%)=498\left(1,[\mathrm{M}]^{+}\right), 480\left(10\left[\mathrm{M}-\mathrm{H}_{2} \mathrm{O}\right]^{+}\right), 333(100$, $\left[\mathrm{C}_{20} \mathrm{H}_{15} \mathrm{NO}_{2} \mathrm{~S}\right]^{+}$), 280 (10), 170 (3), 71 (10).

HRMS (EI): $m / z[M]^{+}$calcd for $\mathrm{C}_{28} \mathrm{H}_{22} \mathrm{~N}_{2} \mathrm{O}_{5} \mathrm{~S}$ : 498.1249; found: 498.1248 .
2-\{(1R,2R)-2-(10'-0xo-10'H-thioxantheno[2',3'-d]oxazol-2'-yl)cyclohexyl\}isoindoline-1",3"-dione (12)

The synthesized amide 11 ( $275 \mathrm{mg}, 552 \mu \mathrm{mol}, 1.00$ equiv) was dissolved in anhyd THF $(26 \mathrm{~mL})$. At $\mathrm{rt}, \mathrm{PPh}_{3}(318 \mathrm{mg}, 1.21 \mathrm{mmol}$, 2.20 equiv) and diisopropyl azodicarboxylate $(245 \mathrm{mg}, 0.24 \mathrm{~mL}$, $1.21 \mathrm{mmol}, 2.20$ equiv) were added. The mixture was stirred at rt and after $4 \mathrm{~h}$, the solvent was evaporated. The crude product was purified by flash chromatography $\left(4 \times 25 \mathrm{~cm}, \mathrm{CH}_{2} \mathrm{Cl}_{2} / \mathrm{MeOH} 98: 2\right)$. The desired product was obtained as a yellow solid ( $220 \mathrm{mg}, 458 \mu \mathrm{mol}, 83 \%)$; mp $225-227^{\circ} \mathrm{C} ; R_{f}=0.50\left(\mathrm{CH}_{2} \mathrm{Cl}_{2} / \mathrm{MeOH} 98: 2\right)$ [UV, $\left.\mathrm{KMnO}_{4}\right] ;[\alpha]_{\mathrm{D}}{ }^{20}-152$ (c = 1.00, $\mathrm{CH}_{2} \mathrm{Cl}_{2}$ ).

IR (ATR): $3071\left(\mathrm{w}, \mathrm{CH}_{\mathrm{sp} 2}\right), 2952\left(\mathrm{~m}, \mathrm{CH}_{\mathrm{sp} 3}\right), 2921\left(\mathrm{~m}, \mathrm{CH}_{\mathrm{sp} 3}\right), 2864(\mathrm{w}$, $\left.\mathrm{CH}_{\mathrm{sp} 3}\right), 1764(\mathrm{~m}, \mathrm{C}=\mathrm{O}), 1701(\mathrm{~s}, \mathrm{C}=\mathrm{O}), 1643(\mathrm{~m}), 1620(\mathrm{~s}), 1435(\mathrm{~m}$, $\left.\mathrm{CH}_{\mathrm{sp} 3}\right), 1394(\mathrm{~m}), 1373(\mathrm{~m}), 1293(\mathrm{~m}), 1244(\mathrm{w}), 1155(\mathrm{w}), 1077(\mathrm{~m})$, $1023(\mathrm{~m}), 719 \mathrm{~cm}^{-1}\left(\mathrm{~m}, \mathrm{CH}_{\mathrm{sp} 2}\right)$.

${ }^{1} \mathrm{H}$ NMR $\left(400 \mathrm{MHz}, \mathrm{CDCl}_{3}\right): \delta=1.49-1.65(\mathrm{~m}, 2 \mathrm{H}, \mathrm{H}-4, \mathrm{H}-5), 1.82-2.07$ ( $\mathrm{m}, 4 \mathrm{H}, \mathrm{H}-3, \mathrm{H}-4, \mathrm{H}-5, \mathrm{H}-6$ ), 2.29-2.45 (m, $2 \mathrm{H}, \mathrm{H}-3, \mathrm{H}-6), 4.12$ (ddd, $\left.{ }^{3} J=12.3 \mathrm{~Hz}, 11.4 \mathrm{~Hz}, 3.6 \mathrm{~Hz}, 1 \mathrm{H}, \mathrm{H}-2\right), 4.57$ (virt. td, ${ }^{3} \mathrm{~J} \cong 3^{3}=11.7 \mathrm{~Hz}$, $\left.{ }^{3} J=3.8 \mathrm{~Hz}, 1 \mathrm{H}, \mathrm{H}-1\right), 7.44\left(\mathrm{ddd},{ }^{3} \mathrm{~J}=8.2 \mathrm{~Hz}, 6.9 \mathrm{~Hz},{ }^{4} \mathrm{~J}=1.3 \mathrm{~Hz}, 1 \mathrm{H}, \mathrm{H}-\right.$ 8'), 7.48-7.52 (m, $\left.2 \mathrm{H}, \mathrm{H}-4^{\prime}, \mathrm{H}-6^{\prime}\right), 7.57$ (ddd, ${ }^{3} \mathrm{~J}=8.3 \mathrm{~Hz}, 6.9 \mathrm{~Hz}$, $\left.{ }^{4} J=1.5 \mathrm{~Hz}, 1 \mathrm{H}, \mathrm{H}-7^{\prime}\right), 7.64\left(\mathrm{dd},{ }^{3} \mathrm{~J}=5.5 \mathrm{~Hz},{ }^{4} \mathrm{~J}=3.0 \mathrm{~Hz}, 2 \mathrm{H}, \mathrm{H}-5^{\prime \prime}, \mathrm{H}-6^{\prime \prime}\right)$, $7.74\left(\mathrm{dd},{ }^{3} \mathrm{~J}=5.5 \mathrm{~Hz},{ }^{4} \mathrm{~J}=3.0 \mathrm{~Hz}, 2 \mathrm{H}, \mathrm{H}-4^{\prime \prime}, \mathrm{H}-7^{\prime \prime}\right), 8.55$ (dd, ${ }^{3} \mathrm{~J}=8.2 \mathrm{~Hz}$, $\left.{ }^{4} J=1.5 \mathrm{~Hz}, 1 \mathrm{H}, \mathrm{H}-9^{\prime}\right), 8.76$ (s, $\left.1 \mathrm{H}, \mathrm{H}-11^{\prime}\right)$.

${ }^{13} \mathrm{C} \mathrm{NMR}\left(101 \mathrm{MHz}, \mathrm{CDCl}_{3}\right): \delta=24.8(\mathrm{t}, \mathrm{C}-4), 25.5(\mathrm{t}, \mathrm{C}-5), 29.9(\mathrm{t}, \mathrm{C}-3)$, 30.7 (t, C-6), 40.0 (d, C-2), 52.5 (d, C-1), 106.3 (d, C-4'), 122.0 (d, C11'), 123.4 (d, 2 C, C-4", C-7"), 125.7 (d, C-6'), 126.4 (d, C-8'), 126.5 (s, C-4a'), 128.7 (s, C-9a'), 130.0 (d, C-9'), 131.8 (s, 2 C, C-3a", C-7a"), 132.3 (d, C-7'), 134.1 (d, 2 C, C-5", C-6"), 134.2 (s, C-10a'), 137.0 (s, C5a'), 141.0 (s, C-3a'), 153.0 (s, C-11a'), 168.2 (s, 2 C, C-1", C-3"), 168.9 (s, C-2'), 179.8 (s, C-10').

HRMS (ESI): $m / z[\mathrm{M}+\mathrm{H}]^{+}$calcd for $\mathrm{C}_{28} \mathrm{H}_{20} \mathrm{~N}_{2} \mathrm{O}_{4} \mathrm{~S}: 481.1217$; found: 481.1217.

1-[3",5"-Bis(trifluoromethyl)phenyl]-3-\{(1R,2R)-2-(10'-oxo-10'Hthioxantheno[2',3'-d]oxazol-2'-yl)cyclohexyl\}thiourea (1)

Oxazole 12 (70.0 mg, $146 \mu \mathrm{mol}, 1.00$ equiv) was dissolved in anhyd $\mathrm{MeOH}(13 \mathrm{~mL})$. Hydrazine hydrate $(364 \mathrm{mg}, 0.36 \mathrm{~mL}, 7.29 \mathrm{mmol}$, 50.0 equiv) was added and the mixture was stirred at rt for $24 \mathrm{~h}$. Upon removal of the solvent, the residue was dissolved in $\mathrm{CH}_{2} \mathrm{Cl}_{2}$ and filtered over $\mathrm{SiO}_{2}\left(1 \times 2 \mathrm{~cm}, \mathrm{CH}_{2} \mathrm{Cl}_{2} / \mathrm{MeOH}\right.$ 95:5). After evaporation of the solvent, the crude material was used in the next step without further purification. The crude material was dissolved in anhyd THF $(10 \mathrm{~mL})$ and 3,5-bis(trifluoromethyl)phenyl isothiocyanate $(83.1 \mathrm{mg}$, $0.06 \mathrm{~mL}, 307 \mu \mathrm{mol}, 2.10$ equiv) was added. The reaction mixture was stirred at rt and after $18 \mathrm{~h}$, the solvent was evaporated. Purification by flash chromatography $\left(2 \times 7 \mathrm{~cm}, \mathrm{CH}_{2} \mathrm{Cl}_{2} / \mathrm{MeOH} 99: 1\right)$ afforded the desired thiourea as a yellow solid $(29.0 \mathrm{mg}, 46.7 \mu \mathrm{mol}, 32 \%$ over two steps); mp 124-126 ${ }^{\circ} \mathrm{C} ; R_{f}=0.20\left(\mathrm{CH}_{2} \mathrm{Cl}_{2} / \mathrm{MeOH} 99: 1\right)$ [UV, $\mathrm{KMnO}_{4}$ ]; $[\alpha]_{\mathrm{D}}^{20}+80\left(c=1.00, \mathrm{CH}_{2} \mathrm{Cl}_{2}\right)$.

IR (ATR): 3327 (w, NH), $2956\left(\mathrm{w}, \mathrm{CH}_{\mathrm{sp} 3}\right), 2929\left(\mathrm{w}, \mathrm{CH}_{\mathrm{sp} 3}\right), 2852(\mathrm{w}$, $\left.\mathrm{CH}_{\mathrm{sp} 3}\right), 1701(\mathrm{~m}, \mathrm{C}=\mathrm{O}), 1625\left(\mathrm{~m}, \mathrm{C}=\mathrm{C}_{\mathrm{sp} 2}\right), 1438\left(\mathrm{~m}, \mathrm{CH}_{\mathrm{sp} 3}\right), 1277(\mathrm{~m})$, $1028(\mathrm{~m}), 910(\mathrm{~m}), 749 \mathrm{~cm}^{-1}\left(\mathrm{~m}, \mathrm{CH}_{\mathrm{sp} 2}\right)$.

${ }^{1} \mathrm{H} \mathrm{NMR}\left(400 \mathrm{MHz}, \mathrm{CDCl}_{3}\right): \delta=1.55-1.79(\mathrm{~m}, 4 \mathrm{H}, \mathrm{H}-4, \mathrm{H}-5), 1.93-2.00$ (m, $2 \mathrm{H}, \mathrm{H}-3, \mathrm{H}-6), 2.08-2.17$ (m, $1 \mathrm{H}, \mathrm{H}-6), 2.37-2.41$ (m, $1 \mathrm{H}, \mathrm{H}-3$ ), 3.73 (br s $1 \mathrm{H}, \mathrm{H}-2$ ), 4.96-5.16 (m, $1 \mathrm{H}, \mathrm{H}-1$ ), 7.41-7.68 (m, $5 \mathrm{H}, \mathrm{H}-4$ ', H-6', H-7', H-8', H-4"), 7.95 (s, $\left.2 \mathrm{H}, \mathrm{H}-2^{\prime \prime}\right), 8.15$ (br s, $1 \mathrm{H}, \mathrm{NH}$ ), 8.50 (d, $\left.3^{3}=8.1 \mathrm{~Hz}, 1 \mathrm{H}, \mathrm{H}-9^{\prime}\right), 8.68$ (s, $\left.1 \mathrm{H}, \mathrm{H}-11^{\prime}\right), 9.01$ (br s, $1 \mathrm{H}, \mathrm{NH}$ ).

${ }^{13} \mathrm{C}$ NMR (101 MHz, $\left.\mathrm{CDCl}_{3}\right)$ : $\delta=21.2\left(\mathrm{t}, \mathrm{C}-4^{*}\right), 22.0\left(\mathrm{t}, \mathrm{C}-5^{*}\right), 28.3(\mathrm{t}, \mathrm{C}-$ 6), 39.3 (t, C-3), 54.0 (d, C-2), 60.6 (d, C-1), 106.8 (d, C-4'), 118.7 (d, C4"), 119.0 (s, C-1"), 121.3 (d, C-11'), 122.5 (q, ${ }^{1} J_{\mathrm{C}, \mathrm{F}}=165 \mathrm{~Hz}, 2 \mathrm{C}, \mathrm{CF}_{3}$ ), 
123.8 (d, 2 C, C-2"), 125.9 (d, C-6'*), 126.3 (d, C-7**), 128.2 (s, C-4a'), 129.9 (d, C-8'), 132.4 (q, ${ }^{2} J=33.6$ Hz, 2 C, C-3"), 132.8 (d, C-9'), 134.9 (s, C-9a'), 137.4 (s, C-5a'), 139.9 (s, C-10a'), 140.0 (s, C-3a'), 152.5 (s, C-11a'), 169.3 (s, C-2'), 180.0 (s, C-10'), 180.3 (s, NCSN).

HRMS (ESI): $m / z[\mathrm{M}+\mathrm{H}]^{+}$calcd for $\mathrm{C}_{29} \mathrm{H}_{21} \mathrm{~F}_{6} \mathrm{~N}_{3} \mathrm{O}_{2} \mathrm{~S}_{2}: 622.1052$; found: 622.1047.

\section{(1S,2S,3R,4R)-3-(1',3'-Dioxoisoindolin-2'-yl)bicyclo[2.2.1]hept-5- ene-2-carboxylic Acid (15)}

Amino acid 14 (962 mg, $6.28 \mathrm{mmol}, 1.00$ equiv) was dissolved in anhyd toluene $(150 \mathrm{~mL})$. Subsequently, phthalic anhydride $(978 \mathrm{mg}$, $6.60 \mathrm{mmol}, 1.05$ equiv) and $\mathrm{NEt}_{3}(1.26 \mathrm{~g}, 1.74 \mathrm{~mL}, 12.6 \mathrm{mmol}$, 2.00 equiv) were added. The mixture was stirred at $100{ }^{\circ} \mathrm{C}$. After $18 \mathrm{~h}$, the solution was cooled to rt and washed with aq $3 \mathrm{M} \mathrm{HCl}(150 \mathrm{~mL})$. The aqueous layer was extracted with $\mathrm{CH}_{2} \mathrm{Cl}_{2}(3 \times 150 \mathrm{~mL})$. The combined organic layers were dried $\left(\mathrm{Na}_{2} \mathrm{SO}_{4}\right)$, filtered, and the solvent was removed under reduced pressure. The desired product was obtained as a brown viscous oil $(1.60 \mathrm{~g}, 5.65 \mathrm{mmol}, 90 \%) ;[\alpha]_{D}{ }^{20}+64(c=1.00$, $\mathrm{CH}_{2} \mathrm{Cl}_{2}$ ).

IR (ATR): 3227 (m, OH), 3059 (w, $\left.\mathrm{CH}_{\mathrm{sp} 2}\right), 2983\left(\mathrm{w}, \mathrm{CH}_{\mathrm{sp} 3}\right), 2952$ (w, $\left.\mathrm{CH}_{\mathrm{sp} 3}\right), 1770(\mathrm{~m}), 1703$ (s, C=0), $1402(\mathrm{~m}), 1367$ (s), 1329 (s), 1178 (m), $1158(\mathrm{~m}), 872(\mathrm{~m}), 714(\mathrm{~s}), 695(\mathrm{~s}), 662 \mathrm{~cm}^{-1}(\mathrm{~m})$.

${ }^{1} \mathrm{H}$ NMR $\left(300 \mathrm{MHz}, \mathrm{CDCl}_{3}\right): \delta=1.75$ (virt. dt, ${ }^{2} \mathrm{~J}=9.3 \mathrm{~Hz},{ }^{3} \mathrm{~J} \cong$ ${ }^{3} J=1.9 \mathrm{~Hz}, 1 \mathrm{H}, \mathrm{H}-7$ ), 2.63 (virt. dt, ${ }^{2} J=9.3 \mathrm{~Hz},{ }^{3} J \cong{ }^{3} J=1.9 \mathrm{~Hz}, 1 \mathrm{H}, \mathrm{H}-$ 7), $2.77\left(\mathrm{dd},{ }^{3} \mathrm{~J}=8.6 \mathrm{~Hz},{ }^{4} \mathrm{~J}=1.8 \mathrm{~Hz}, 1 \mathrm{H}, \mathrm{H}-2\right.$ ), 3.18 (virt. $\mathrm{t},{ }^{3} \mathrm{~J} \cong$ $\left.{ }^{3} J=1.9 \mathrm{~Hz}, 1 \mathrm{H}, \mathrm{H}-4\right), 3.42\left(\mathrm{~d},{ }^{3} J=1.9 \mathrm{~Hz}, 1 \mathrm{H}, \mathrm{H}-1\right), 4.28$ (dd, $\left.{ }^{3} J=8.6 \mathrm{~Hz},{ }^{4} J=1.8 \mathrm{~Hz}, 1 \mathrm{H}, \mathrm{H}-3\right), 6.22\left(\mathrm{dd},{ }^{3} J=5.6 \mathrm{~Hz},{ }^{3} J=2.9 \mathrm{~Hz}, 1 \mathrm{H}\right.$, H-6), $6.31\left(\mathrm{dd},{ }^{3} \mathrm{~J}=5.6 \mathrm{~Hz},{ }^{3} \mathrm{~J}=3.1 \mathrm{~Hz}, 1 \mathrm{H}, \mathrm{H}-5\right), 7.64-7.70(\mathrm{~m}, 2 \mathrm{H}, \mathrm{H}-$ 5', H-6'), 7.74-7.81 (m, $\left.2 \mathrm{H}, \mathrm{H}-4^{\prime}, \mathrm{H}-7^{\prime}\right)$.

${ }^{13} \mathrm{C}$ NMR (75 MHz, $\mathrm{CDCl}_{3}$ ): $\delta=45.3$ (d, C-1), 45.6 (d, C-2), 45.9 (d, C-4), 47.8 (t, C-7), 55.6 (d, C-3), 123.2 (d, 2 C, C-4', C-7'), 132.0 (s, 2 C, C-3a', C-7a'), 134.0 (d, 2 C, C-5', C-6'), 138.3 (d, C-5), 138.8 (d, C-6), 168.9 (s, 2 C, C-1', C-3'), 176.9 (s, COOH).

MS (EI, $70 \mathrm{eV}): m / z(\%)=92\left(60,\left[\mathrm{C}_{7} \mathrm{H}_{8}\right]^{+}\right), 91(100), 65$ (13), 40 (12, $\left.\left[\mathrm{C}_{3} \mathrm{H}_{4}\right]^{+}\right)$.

HRMS (EI): $m / z$ [M] $]^{+}$calcd for $\mathrm{C}_{16} \mathrm{H}_{13} \mathrm{NO}_{4}$ : 283.0845; found: 283.0836 .

\section{(1R,2S,3R,4S)-3-(1',3'-Dioxoisoindolin-2'-yl)bicyclo[2.2.1]heptane-} 2-carboxylic Acid (16)

The protected amino acid 15 ( $1.53 \mathrm{~g}, 5.42 \mathrm{mmol}, 1.00$ equiv) was dissolved in EtOAc $(92 \mathrm{~mL})$ and $\mathrm{Pd} / \mathrm{C}(10 \% \mathrm{w} / \mathrm{w}, 181 \mathrm{mg})$ was added. The mixture was stirred under $\mathrm{H}_{2}$ atmosphere (balloon) at rt. After $18 \mathrm{~h}$, the mixture was filtered over Celite and washed with $\mathrm{CH}_{2} \mathrm{Cl}_{2}$ $(150 \mathrm{~mL})$. The solvent was evaporated under reduced pressure. The product was isolated as a colorless solid $(1.51 \mathrm{~g}, 5.31 \mathrm{mmol}, 98 \%)$; mp $75-78{ }^{\circ} \mathrm{C} ;[\alpha]_{\mathrm{D}}^{20}+36\left(c=1.00, \mathrm{CH}_{2} \mathrm{Cl}_{2}\right)$.

Chiral HPLC (OD-RH, $150 \times 4.6 \mathrm{~mm}, \mathrm{MeCN} / \mathrm{H}_{2} \mathrm{O}(2: 10), 1 \mathrm{~mL} / \mathrm{min}$, $\lambda=210 \mathrm{~nm}, 254 \mathrm{~nm}): t_{\mathrm{R} 1}=11.1 \mathrm{~min}, t_{\mathrm{R} 2}=12.9 \mathrm{~min}$.

IR (ATR): $3258(\mathrm{~m}, \mathrm{OH}), 2959\left(\mathrm{~m}, \mathrm{CH}_{\mathrm{sp} 3}\right), 2874\left(\mathrm{~m}, \mathrm{CH}_{\mathrm{sp} 3}\right), 1731(\mathrm{~s}$, $\mathrm{C}=0), 1453(\mathrm{w}), 1373(\mathrm{w}), 1286(\mathrm{~m}), 1107(\mathrm{~m}), 1073(\mathrm{w}), 719 \mathrm{~cm}^{-1}(\mathrm{~s})$. ${ }^{1} \mathrm{H}$ NMR $\left(400 \mathrm{MHz}, \mathrm{CDCl}_{3}\right): \delta=1.24-1.38\left(\mathrm{~m}, 3 \mathrm{H}, \mathrm{H}-5_{a x}, \mathrm{H}-6_{a x}, \mathrm{H}-7\right)$, 1.60-1.71 (m, $2 \mathrm{H}, \mathrm{H}-5_{e q}, \mathrm{H}-6_{e q}$ ), 2.61 (virt. dt, ${ }^{2} \mathrm{~J}=10.8 \mathrm{~Hz},{ }^{3} \mathrm{~J} \cong$ $3 J=2.1 \mathrm{~Hz}, 1 \mathrm{H}, \mathrm{H}-7), 2.67\left(\mathrm{~d},{ }^{3} \mathrm{~J}=3.7 \mathrm{~Hz}, 1 \mathrm{H}, \mathrm{H}-1^{*}\right), 2.77$ (d, $\left.{ }^{3} \mathrm{~J}=3.4 \mathrm{~Hz}, 1 \mathrm{H}, \mathrm{H}-4^{*}\right), 2.86\left(\mathrm{dd},{ }^{3} \mathrm{~J}=9.3 \mathrm{~Hz},{ }^{4} \mathrm{~J}=1.7 \mathrm{~Hz}, 1 \mathrm{H}, \mathrm{H}-2\right), 4.38$ $\left(\mathrm{dd},{ }^{3} \mathrm{~J}=9.3 \mathrm{~Hz},{ }^{4} \mathrm{~J}=1.5 \mathrm{~Hz}, 1 \mathrm{H}, \mathrm{H}-3\right), 7.69\left(\mathrm{dd},{ }^{3} \mathrm{~J}=5.5 \mathrm{~Hz},{ }^{4} \mathrm{~J}=3.0 \mathrm{~Hz}, 2\right.$ H, H-5', H-6'), 7.80 (dd, ${ }^{3} J=5.5 \mathrm{~Hz},{ }^{4} J=3.0 \mathrm{~Hz}, 2 \mathrm{H}, \mathrm{H}-4^{\prime}, \mathrm{H}-7^{\prime}$ ).
${ }^{13} \mathrm{C}$ NMR (101 MHz, CDCl $)$ ): $\delta=28.0(\mathrm{t}, \mathrm{C}-6), 29.8(\mathrm{t}, \mathrm{C}-5), 37.8(\mathrm{t}, \mathrm{C}-7)$, $40.1\left(\mathrm{~d}, \mathrm{C}-1^{*}\right), 40.2\left(\mathrm{~d}, \mathrm{C}-4^{*}\right), 53.1$ (d, C-2), 57.5 (d, C-3), 123.3 (d, 2 C, C-4', C-7'), 132.0 (s, 2 C, C-3a', C-7a'), 134.0 (d, 2 C, C-5', C-6'), 168.7 (s, $\left.2 \mathrm{C}, \mathrm{C}-1^{\prime}, \mathrm{C}-3^{\prime}\right), 175.1$ (s, $\left.\mathrm{COOH}\right)$.

MS (EI, $70 \mathrm{eV}): m / z(\%)=185\left(1,[\mathrm{M}]^{+}\right), 94(21), 78(9), 66(100), 40$ (10).

HRMS (EI): $m / z$ [M] calcd for $\mathrm{C}_{16} \mathrm{H}_{15} \mathrm{NO}_{4}$ : 285.0996; found: 285.1000 .

2'-Nitro-9'-oxo-9'H-thioxanthen-3'-yl (1R,2S,3R,4S)-3-(1",3"-Dioxoisoindolin-2"-yl)bicyclo[2.2.1]heptane-2-carboxylate (17)

The protected amino acid 16 (870 mg, $3.05 \mathrm{mmol}, 1.10$ equiv) was dissolved in anhyd $\mathrm{CH}_{2} \mathrm{Cl}_{2}(30 \mathrm{~mL})$, and oxalyl chloride $(387 \mathrm{mg}$, $0.26 \mathrm{~mL}, 3.05 \mathrm{mmol}, 1.10$ equiv) and a catalytic amount of DMF (5 drops) was added. The mixture was stirred at rt for $3 \mathrm{~h}$. Meanwhile thioxanthone $\mathbf{9}$ ( $757 \mathrm{mg}, 2.77 \mathrm{mmol}, 1.00$ equiv) was dissolved in anhyd $\mathrm{CH}_{2} \mathrm{Cl}_{2}(65 \mathrm{~mL})$ and cooled to $0{ }^{\circ} \mathrm{C}$. At this temperature, $\mathrm{NEt}_{3}$ (854 mg, $1.17 \mathrm{~mL}, 8.31 \mathrm{mmol}, 3.00$ equiv) and a catalytic amount of 4-dimethylaminopyridine ( 5 crystals) was added. At $0{ }^{\circ} \mathrm{C}$ the freshly prepared solution of acid chloride was added slowly. After addition, the mixture was slowly warmed to $\mathrm{rt}$ and stirred overnight. After 18 $\mathrm{h}$, the reaction was stopped with aq $\mathrm{NH}_{4} \mathrm{Cl}(100 \mathrm{~mL})$ and extracted with $\mathrm{CH}_{2} \mathrm{Cl}_{2}(3 \times 100 \mathrm{~mL})$. The combined organic layers were washed with aq $2 \mathrm{M} \mathrm{NaOH}(150 \mathrm{~mL})$, dried $\left(\mathrm{Na}_{2} \mathrm{SO}_{4}\right)$, and filtered. The solvent was evaporated under reduced pressure and the product was obtained as a yellow solid (1.48 g, $2.74 \mathrm{mmol}, 99 \%) ; \mathrm{mp} \mathrm{202-206}{ }^{\circ} \mathrm{C}$; $R_{f}=0.83\left(\mathrm{CH}_{2} \mathrm{Cl}_{2} / \mathrm{MeOH}\right.$ 98:2) [UV, $\left.\mathrm{KMnO}_{4}\right] ;[\alpha]_{\mathrm{D}}^{20}-92(c=1.00$, $\mathrm{CH}_{2} \mathrm{Cl}_{2}$ ).

IR (ATR): $3089\left(\mathrm{w}, \mathrm{CH}_{\mathrm{sp} 2}\right), 2956\left(\mathrm{w}, \mathrm{CH}_{\mathrm{sp} 3}\right), 2880\left(\mathrm{w}, \mathrm{CH}_{\mathrm{sp} 3}\right), 1759$ (s, $\mathrm{C}=0), 1702(\mathrm{~s}, \mathrm{C}=\mathrm{O}), 1640\left(\mathrm{~s}, \mathrm{C}=\mathrm{C}_{\mathrm{sp} 2}\right), 1338(\mathrm{~s}, \mathrm{~N}=\mathrm{O}), 1105(\mathrm{~s}, \mathrm{C}-\mathrm{O}), 730$ $\mathrm{cm}^{-1}(\mathrm{~m}, \mathrm{C}-\mathrm{S})$.

${ }^{1} \mathrm{H}$ NMR $\left(400 \mathrm{MHz}, \mathrm{CDCl}_{3}\right): \delta=1.45\left(\mathrm{~d},{ }^{3} \mathrm{~J}=2.3 \mathrm{~Hz}, 1 \mathrm{H}, \mathrm{H}-6_{a x}\right), 1.47(\mathrm{~d}$, $\left.3 J=2.3 \mathrm{~Hz}, 1 \mathrm{H}, \mathrm{H}-5_{a x}\right), 1.52-1.55(\mathrm{~m}, 1 \mathrm{H}, \mathrm{H}-7), 1.71-1.86(\mathrm{~m}, 2 \mathrm{H}, \mathrm{H}-$ $\left.5_{e q}, \mathrm{H}-6_{e q}\right), 2.72$ (virt. dt, ${ }^{2} \mathrm{~J}=10.9 \mathrm{~Hz},{ }^{3} \mathrm{~J} \cong 3^{3} \mathrm{~J}=2.0 \mathrm{~Hz}, 1 \mathrm{H}, \mathrm{H}-7$ ), $2.94(\mathrm{~s}$, $\left.1 \mathrm{H}, \mathrm{H}-1^{*}\right), 3.01\left(\mathrm{~s}, 1 \mathrm{H}, \mathrm{H}-4^{*}\right), 3.31\left(\mathrm{dd},{ }^{3} \mathrm{~J}=9.1 \mathrm{~Hz},{ }^{4} \mathrm{~J}=1.6 \mathrm{~Hz}, 1 \mathrm{H}, \mathrm{H}-\right.$ 2), 4.53 (dd, $\left.{ }^{3} \mathrm{~J}=9.1 \mathrm{~Hz},{ }^{4} \mathrm{~J}=1.5 \mathrm{~Hz}, 1 \mathrm{H}, \mathrm{H}-3\right), 7.05$ (s, $\left.1 \mathrm{H}, \mathrm{H}-4^{\prime}\right), 7.50-$ 7.57 (m, 2 H, H-5', H-7'), 7.64-7.74 (m, 3 H, H-6', H-5", H-6"), 7.84 (dd, $\left.3^{3}=5.4 \mathrm{~Hz},{ }^{4} J=3.0 \mathrm{~Hz}, 2 \mathrm{H}, \mathrm{H}-4^{\prime \prime}, \mathrm{H}-7^{\prime \prime}\right), 8.55\left(\mathrm{dd}, 3^{3}=8.0 \mathrm{~Hz}\right.$, $\left.{ }^{4} J=1.5 \mathrm{~Hz}, 1 \mathrm{H}, \mathrm{H}-8^{\prime}\right), 9.19$ (s, $\left.1 \mathrm{H}, \mathrm{H}-1^{\prime}\right)$.

${ }^{13} \mathrm{C}$ NMR (101 MHz, CDCl $): \delta=28.4(\mathrm{t}, \mathrm{C}-6), 29.6(\mathrm{t}, \mathrm{C}-5), 37.9(\mathrm{t}, \mathrm{C}-7)$, $40.1\left(\mathrm{~d}, \mathrm{C}-1^{*}\right), 40.5\left(\mathrm{~d}, \mathrm{C}-4^{*}\right), 53.6(\mathrm{~d}, \mathrm{C}-2), 58.0(\mathrm{~d}, \mathrm{C}-3), 122.0\left(\mathrm{~d}, \mathrm{C}-4^{\prime}\right)$, 123.4 (d, 2 C, C-4", C-7"), 126.2 (d, C-5'), 127.0 (s, C-9a'), 127.8 (d, C$\left.7^{\prime}\right), 128.3$ (d, C-1'), 128.5 (s, C-8a'), 130.3 (d, C-8'), 132.0 (s, 2 C, C-3a", C-7a"), 133.4 (d, C-6'), 134.3 (d, 2 C, C-5", C-6"), 135.7 (s, C-4b'), 140.1 (s, C-3'), 144.1 (s, C-4a'), 146.0 (s, C-2'), 168.7 (s, 2 C, C-1"', C-3"), 169.0 (s, COO), $177.9\left(\mathrm{~s}, \mathrm{C}-9^{\prime}\right)$.

MS (EI, $70 \mathrm{eV}): m / z(\%)=268$ (24), 239 (100), 211 (65), 200 (40), 186 (25), 148 (28), 92 (26), 57 (23).

HRMS (EI): $m / z[M]^{+}$calcd for $\mathrm{C}_{29} \mathrm{H}_{20} \mathrm{~N}_{2} \mathrm{O}_{7} \mathrm{~S}$ : 540.0986; found: 540.0993.

(1R,2S,3R,4S)-3-(1",3"--Dioxoisoindolin-2"'-yl)-N-(3'-hydroxy-9'oxo-9' $H$-thioxanthen-2'-yl)bicyclo[2.2.1] heptane-2-carboxamide (18)

The ester 17 ( $400 \mathrm{mg}, 741 \mu \mathrm{mol}, 1.00$ equiv) was dissolved in THF $(26 \mathrm{~mL})$ and $\mathrm{H}_{2} \mathrm{O}(26 \mathrm{~mL})$ was added. In $(425 \mathrm{mg}, 3.70 \mathrm{mmol}$, 5.00 equiv) and concd $\mathrm{HCl}(0.72 \mathrm{~mL})$ were added. The mixture was heated to $80^{\circ} \mathrm{C}$ and stirred for $20 \mathrm{~h}$. After cooling to $\mathrm{rt}, \mathrm{CH}_{2} \mathrm{Cl}_{2}$ $(100 \mathrm{~mL})$ was added and the mixture was filtered over Celite. The mixture was washed with aq $\mathrm{NaHCO}_{3}(150 \mathrm{~mL})$ and extracted with $\mathrm{CH}_{2} \mathrm{Cl}_{2}(3 \times 100 \mathrm{~mL})$. The combined organic layers were dried 
$\left(\mathrm{Na}_{2} \mathrm{SO}_{4}\right)$, filtered, and evaporated. The crude material was purified by flash column chromatography $\left(4 \times 20 \mathrm{~cm}, \mathrm{CH}_{2} \mathrm{Cl}_{2} / \mathrm{MeOH} 95: 5\right)$ and the product was isolated as an orange solid (328 $\mathrm{mg}, 642 \mu \mathrm{mol}, 87 \%$ ); mp 166-169 ${ }^{\circ} \mathrm{C} ; R_{f}=0.44\left(\mathrm{CH}_{2} \mathrm{Cl}_{2} / \mathrm{MeOH} 95: 5\right)$ [UV, $\left.\mathrm{KMnO}_{4}\right] ;[\alpha]_{\mathrm{D}}{ }^{20}$ $-144\left(c=1.00, \mathrm{CH}_{2} \mathrm{Cl}_{2}\right)$.

IR (ATR): $3248(\mathrm{~m}, \mathrm{OH}), 3141(\mathrm{~m}, \mathrm{NH}), 2967\left(\mathrm{w}, \mathrm{CH}_{\mathrm{sp} 3}\right), 2872(\mathrm{w}$, $\left.\mathrm{CH}_{\mathrm{sp} 3}\right), 1697$ (s, C=O), 1583 (s, C=C $\left.\mathrm{C}_{\mathrm{sp} 2}\right), 1304(\mathrm{~s}, \mathrm{C}-\mathrm{N}), 718 \mathrm{~cm}^{-1}(\mathrm{~s}, \mathrm{C}-\mathrm{S})$. ${ }^{1} \mathrm{H}$ NMR $\left(400 \mathrm{MHz}, \mathrm{CDCl}_{3}\right): \delta=1.18-1.38\left(\mathrm{~m}, 2 \mathrm{H}, \mathrm{H}-5_{a x}, \mathrm{H}-6_{a x}\right), 1.54-$ 1.60 ( $\mathrm{m}, 1 \mathrm{H}, \mathrm{H}-7), 1.65-1.80$ ( $\left.\mathrm{m}, 2 \mathrm{H}, \mathrm{H}-5_{\text {eq }}, \mathrm{H}-6_{e q}\right), 2.68$ (s, $\left.1 \mathrm{H}, \mathrm{H}-1\right)$, $2.85\left(\mathrm{~d},{ }^{2} \mathrm{~J}=11.1 \mathrm{~Hz}, 1 \mathrm{H}, \mathrm{H}-7\right), 3.08-3.15(\mathrm{~m}, 2 \mathrm{H}, \mathrm{H}-2, \mathrm{H}-4), 4.44$ (dd, $\left.{ }^{3} J=8.6 \mathrm{~Hz},{ }^{4} \mathrm{~J}=1.6 \mathrm{~Hz}, 1 \mathrm{H}, \mathrm{H}-3\right), 6.94\left(\mathrm{~s}, 1 \mathrm{H}, \mathrm{H}-4^{\prime}\right), 7.44-7.55(\mathrm{~m}, 2 \mathrm{H}$, H-5', H-7'), 7.55-7.62 (m, 3 H, H-6', H-5", H-6"), 7.76 (dd, ${ }^{3} J=5.4 \mathrm{~Hz}$, $\left.{ }^{4} J=3.1 \mathrm{~Hz}, 2 \mathrm{H}, \mathrm{H}-4^{\prime \prime}, \mathrm{H}-7^{\prime \prime}\right), 8.26$ (s, $\left.1 \mathrm{H}, \mathrm{H}-1^{\prime}\right), 8.47$ (s, $\left.1 \mathrm{H}, \mathrm{OH}\right), 8.65$ (dd, $\left.{ }^{3} J=8.2 \mathrm{~Hz},{ }^{4} J=1.5 \mathrm{~Hz}, 1 \mathrm{H}, \mathrm{H}-8^{\prime}\right), 10.11(\mathrm{~s}, 1 \mathrm{H}, \mathrm{NH})$.

${ }^{13} \mathrm{C}$ NMR (101 MHz, CDCl $)$ ): $\delta=28.8$ (t, C-5), 29.3 (t, C-6), 38.4 (t, C-7), 38.7 (d, C-4), 40.9 (d, C-1), 56.0 (d, C-2), 60.0 (d, C-3), 115.2 (d, C-4'), 122.2 (s, C-4a'), 123.5 (d, 2 C, C-4", C-7"), 123.9 (d, C-1'), 126.3 (d, C$\left.7^{\prime}\right), 126.3$ (d, C-5'), 127.3 (s, C-3'), 128.8 (s, C-8a'), 129.9 (d, C-8'), 131.6 (s, 2 C, C-3a", C-7a"), 132.2 (d, C-6'), 134.2 (d, 2 C, C-5", C-6"), 136.7 (s, C-9a'), 137.4 (s, C-4b'), 153.9 (s, C-2'), 169.6 (s, 2 C, C-1", C3"), 173.3 (s, CON), 179.0 (s, C-9').

MS (EI, $70 \mathrm{eV}): m / z(\%)=205(25), 91\left(28,\left[\mathrm{C}_{6} \mathrm{H}_{3} \mathrm{O}\right]^{+}\right), 72(45), 71(49$, $\left.\left[\mathrm{C}_{3} \mathrm{H}_{2} \mathrm{NO}\right]^{+}\right), 42(100)$.

HRMS (EI): $m / z$ [M] $]^{+}$calcd for $\mathrm{C}_{29} \mathrm{H}_{22} \mathrm{~N}_{2} \mathrm{O}_{5} \mathrm{~S}$ : 510.1249; found: 510.1244 .

\section{$2-\left\{(1 S, 2 R, 3 S, 4 R)-3-\left(10^{\prime}-0 \times 0-10^{\prime} H\right.\right.$-thioxantheno[2',3'-d]oxazol-2'- yl)bicyclo[2.2.1] heptan-2-yl\}isoindoline-1",3"-dione (19)}

The amide 18 (523 mg, $1.03 \mathrm{mmol}, 1.00$ equiv) was dissolved in anhyd THF (73 mL), and $\mathrm{PPh}_{3}$ ( $323 \mathrm{mg}, 1.23 \mathrm{mmol}, 1.20$ equiv) and diisopropyl azodicarboxylate ( $249 \mathrm{mg}, 0.24 \mathrm{~mL}, 1.23 \mathrm{mmol}, 1.20$ equiv) were added. The mixture was stirred at rt. After $4 \mathrm{~h}$, the solvent was evaporated and the crude material was purified by flash column chromatography ( $\left.4 \times 25 \mathrm{~cm}, \mathrm{CH}_{2} \mathrm{Cl}_{2} / \mathrm{MeOH} 98: 2\right)$. The product was isolated as a yellow solid (444 mg, $902 \mu \mathrm{mol}, 88 \%) ; \mathrm{mp} \mathrm{223-226}{ }^{\circ} \mathrm{C}$; $R_{f}=0.47\left(\mathrm{CH}_{2} \mathrm{Cl}_{2} / \mathrm{MeOH} 98: 2\right)\left[\mathrm{UV}, \mathrm{KMnO}_{4}\right] ;[\alpha]_{\mathrm{D}}^{20}+30 \quad(c=1.00$, $\left.\mathrm{CH}_{2} \mathrm{Cl}_{2}\right)$.

IR (ATR): 3059 (w, $\left.\mathrm{CH}_{\mathrm{sp} 2}\right), 2991\left(\mathrm{w}, \mathrm{CH}_{\mathrm{sp} 3}\right), 2948\left(\mathrm{w}, \mathrm{CH}_{\mathrm{sp} 3}\right), 2876$ (w, $\left.\mathrm{CH}_{\mathrm{sp} 3}\right), 1766(\mathrm{~m}), 1707(\mathrm{~s}, \mathrm{CO}), 1618(\mathrm{~s}, \mathrm{C}=\mathrm{N}), 1590\left(\mathrm{~m}, \mathrm{C}=\mathrm{C}_{\mathrm{sp} 2}\right), 1435$ (s), $1293(\mathrm{~s}), 1100(\mathrm{~m}), 743(\mathrm{~m}), 714 \mathrm{~cm}^{-1}(\mathrm{~s}, \mathrm{C}-\mathrm{S})$.

${ }^{1} \mathrm{H}$ NMR $\left(400 \mathrm{MHz}, \mathrm{CDCl}_{3}\right): \delta=1.51\left(\mathrm{~d},{ }^{3} \mathrm{~J}=2.3 \mathrm{~Hz}, 1 \mathrm{H}, \mathrm{H}-5\right), 1.52-$ $1.54(\mathrm{~m}, 1 \mathrm{H}, \mathrm{H}-6), 1.61$ (virt. dt, ${ }^{2} J=10.7 \mathrm{~Hz},{ }^{3} \cong{ }^{3} \mathrm{~J}=1.6 \mathrm{~Hz}, 1 \mathrm{H}, \mathrm{H}-7$ ), 1.77-1.90 (m, $\left.2 \mathrm{H}, \mathrm{H}-5_{e q}, \mathrm{H}-6_{e q}\right), 2.87$ (virt. dt, ${ }^{2} \mathrm{~J}=10.7 \mathrm{~Hz},{ }^{3} \cong$ $\left.{ }^{3} \mathrm{~J}=2.0 \mathrm{~Hz}, 1 \mathrm{H}, \mathrm{H}-7\right), 2.96\left(\mathrm{~d},{ }^{3} \mathrm{~J}=3.6 \mathrm{~Hz}, 1 \mathrm{H}, \mathrm{H}-4\right), 3.01\left(\mathrm{~d},{ }^{3} \mathrm{~J}=2.4 \mathrm{~Hz}\right.$, $1 \mathrm{H}, \mathrm{H}-1$ ), 3.61 (dd, ${ }^{3} \mathrm{~J}=8.8 \mathrm{~Hz},{ }^{4} \mathrm{~J}=1.5 \mathrm{~Hz}, 1 \mathrm{H}, \mathrm{H}-3$ ), 4.61 (dd, $\left.{ }^{3} J=8.8 \mathrm{~Hz},{ }^{4} \mathrm{~J}=1.5 \mathrm{~Hz}, 1 \mathrm{H}, \mathrm{H}-2\right), 7.39\left(\mathrm{~s}, 1 \mathrm{H}, \mathrm{H}-4^{\prime}\right), 7.43$ (dd, $\left.{ }^{3} J=8.2 \mathrm{~Hz}, 7.0 \mathrm{~Hz},{ }^{4} \mathrm{~J}=1.3 \mathrm{~Hz}, 1 \mathrm{H}, \mathrm{H}-8^{\prime}\right), 7.50\left(\mathrm{dd},{ }^{3} \mathrm{~J}=8.1 \mathrm{~Hz}\right.$, $\left.{ }^{4} J=1.5 \mathrm{~Hz}, 1 \mathrm{H}, \mathrm{H}-6^{\prime}\right), 7.54-7.61$ (m, $\left.3 \mathrm{H}, \mathrm{H}-7^{\prime}, \mathrm{H}-5^{\prime \prime}, \mathrm{H}-6^{\prime \prime}\right), 7.63-7.67$ (m, 2 H, H-4", H-7"), 8.51-8.56 (m, $\left.1 \mathrm{H}, \mathrm{H}-9^{\prime}\right), 8.59$ (s, $\left.1 \mathrm{H}, \mathrm{H}-11^{\prime}\right)$.

${ }^{13} \mathrm{C}$ NMR (101 MHz, $\mathrm{CDCl}_{3}$ ): $\delta=28.5$ (t, C-5), 30.0 (t, C-6), 38.0 (t, C-7), 39.8 (d, C-4), 41.3 (d, C-1), 49.1 (d, C-3), 58.8 (d, C-2), 106.3 (d, C-4'), 121.8 (d, C-11'), 123.1 (d, 2 C, C-4", C-7"), 125.6 (d, C-6'), 126.4 (d, C8'), 126.4 (s, C-4a'), 128.7 (s, C-9a'), 130.0 (s, C-9'), 131.8 (s, 2 C, C-3a", C-7a"), 132.3 (d, C-7'), 134.0 (d, 2 C, C-5", C-6"), 137.0 (s, C-5a'), 137.3 (s, C-10a'), 141.1 (s, C-3a'), 152.6 (s, C-11a'), 167.2 (s, 2 C, C-1", C-3"), $168.6\left(\mathrm{~s}, \mathrm{C}-2^{\prime}\right), 179.8\left(\mathrm{~s}, \mathrm{C}-10^{\prime}\right)$.

MS (EI, $70 \mathrm{eV}): m / z(\%)=492\left(1,[\mathrm{M}]^{+}\right), 345\left(36,\left[\mathrm{C}_{21} \mathrm{H}_{15} \mathrm{NO}_{2} \mathrm{~S}\right]^{+}\right), 317$ (100, $\left.\left[\mathrm{C}_{19} \mathrm{H}_{11} \mathrm{NO}_{2} \mathrm{~S}\right]^{+}\right), 289(8), 260$ (5), 219 (5), 170 (6).
HR-MS (EI): $\mathrm{m} / \mathrm{z}[\mathrm{M}]^{+}$calcd for $\mathrm{C}_{29} \mathrm{H}_{20} \mathrm{NO}_{4} \mathrm{~S}$ : 492.1144; found: 492.1143.

1-[3",5"-Bis(trifluoromethyl)phenyl]-3-\{(1S,2R,3S,4R)-3-(10'-oxo10'H-thioxantheno[2',3'-d]oxazol-2'-yl)bicyclo[2.2.1] heptan-2yl)thiourea (2)

The oxazole 19 (70.0 mg, $142 \mu \mathrm{mol}, 1.00$ equiv) was suspended in anhyd $\mathrm{EtOH}(4.5 \mathrm{~mL})$, and anhyd $\mathrm{CH}_{2} \mathrm{Cl}_{2}(4.5 \mathrm{~mL})$ was added. To the solution ethylenediamine ( $85.0 \mathrm{mg}, 0.10 \mathrm{~mL}, 1.42 \mathrm{mmol}, 10.0$ equiv) was added. The mixture was warmed to $50{ }^{\circ} \mathrm{C}$ and stirred for $20 \mathrm{~h}$. After cooling, the solvent was evaporated and the crude material was filtered by short flash column chromatography $\left(1 \times 2 \mathrm{~cm}, \mathrm{CH}_{2} \mathrm{Cl}_{2} / \mathrm{MeOH}\right.$ 95:5). The isolated product was used in the next step without further purification. The readily prepared amine was dissolved in anhyd THF $(8 \mathrm{~mL})$ and isothiocyanate 13 ( $80.8 \mathrm{mg}, 54.0 \mu \mathrm{L}, 298 \mu \mathrm{mol}, 2.10$ equiv) was added. The mixture was stirred at rt. After $18 \mathrm{~h}$, the solvent was evaporated and the crude material was purified by flash column chromatography ( $\left.1 \times 5 \mathrm{~cm}, \mathrm{CH}_{2} \mathrm{Cl}_{2} / \mathrm{MeOH} 99: 1\right)$. The product was isolated as a yellow solid ( $35.1 \mathrm{mg}, 55.4 \mu \mathrm{mol}, 39 \%$ over 2 steps); mp 202$204{ }^{\circ} \mathrm{C} ; R_{f}=0.21\left(\mathrm{CH}_{2} \mathrm{Cl}_{2} / \mathrm{MeOH}\right.$ 99:1) [UV, $\left.\mathrm{KMnO}_{4}\right] ;[\alpha]_{\mathrm{D}}^{20}-40$ (c $=1.00, \mathrm{CH}_{2} \mathrm{Cl}_{2}$ ).

IR (ATR): 3281 (m, NH), $3063\left(\mathrm{w}, \mathrm{CH}_{\mathrm{sp} 2}\right), 2960\left(\mathrm{w}, \mathrm{CH}_{\mathrm{sp} 3}\right), 2925$ (w, $\left.\mathrm{CH}_{\mathrm{sp} 3}\right), 2876\left(\mathrm{w}, \mathrm{CH}_{\mathrm{sp} 3}\right), 1766(\mathrm{w}), 1719(\mathrm{~s}, \mathrm{C}=\mathrm{O}), 1637(\mathrm{~m}), 1618(\mathrm{~s}$, $\mathrm{C}=\mathrm{N}), 1591(\mathrm{~m}), 1435(\mathrm{~s}), 1370(\mathrm{~m}), 1275(\mathrm{~s}, \mathrm{C}=\mathrm{S}), 1173(\mathrm{~m}), 1132(\mathrm{~s})$, $1110(\mathrm{~m}), 715 \mathrm{~cm}^{-1}(\mathrm{~m}, \mathrm{C}-\mathrm{S})$.

${ }^{1} \mathrm{H}$ NMR (400 MHz, $\mathrm{CDCl}_{3}$ ): $\delta=1.60-1.79$ (m, $\left.5 \mathrm{H}, \mathrm{H}-5, \mathrm{H}-6, \mathrm{H}-7\right), 1.94$ (d, $\left.{ }^{2} J=11.4 \mathrm{~Hz}, 1 \mathrm{H}, \mathrm{H}-7\right), 2.58$ (s, $\left.1 \mathrm{H}, \mathrm{H}-4\right), 3.13$ (s, $1 \mathrm{H}, \mathrm{H}-1$ ), 3.37 (s, $1 \mathrm{H}, \mathrm{H}-3), 4.32(\mathrm{~s}, 1 \mathrm{H}, \mathrm{H}-2), 6.67(\mathrm{~s}, 1 \mathrm{H}, \mathrm{NH}), 7.52$ (ddd, ${ }^{3} \mathrm{~J}=8.2 \mathrm{~Hz}$, $\left.7.0 \mathrm{~Hz},{ }^{4} \mathrm{~J}=1.3 \mathrm{~Hz}, 1 \mathrm{H}, \mathrm{H}-8^{\prime}\right), 7.58\left(\mathrm{dd},{ }^{3} \mathrm{~J}=7.8 \mathrm{~Hz},{ }^{4} \mathrm{~J}=1.3 \mathrm{~Hz}, 1 \mathrm{H}, \mathrm{H}-\right.$ 6'), 7.62-7.68 (m, $\left.1 \mathrm{H}, \mathrm{H}-7^{\prime}\right), 7.67$ (s, $\left.1 \mathrm{H}, \mathrm{H}-4^{\prime}\right), 7.76$ (s, $\left.1 \mathrm{H}, \mathrm{H}-4^{\prime \prime}\right), 8.23$ (s, $\left.2 \mathrm{H}, \mathrm{H}-2^{\prime \prime}\right), 8.67\left(\mathrm{dd},{ }^{3} \mathrm{~J}=8.2 \mathrm{~Hz},{ }^{4} \mathrm{~J}=1.4 \mathrm{~Hz}, 1 \mathrm{H}, \mathrm{H}-9^{\prime}\right), 8.92(\mathrm{~s}, 1 \mathrm{H}$, $\left.\mathrm{H}-11^{\prime}\right), 10.40$ (s, $\left.1 \mathrm{H}, \mathrm{NH}\right)$.

${ }^{13} \mathrm{C} \mathrm{NMR}\left(126 \mathrm{MHz}, \mathrm{CDCl}_{3}\right): \delta=28.8(\mathrm{t}, \mathrm{C}-5), 29.5(\mathrm{t}, \mathrm{C}-6), 29.9(\mathrm{t}, \mathrm{C}-7)$, 37.5 (d, C-4), 43.6 (d, C-1), 61.3 (d, C-3), 63.3 (d, C-2), 107.4 (d, C-4"), 116.5 (d, C-4'), 118.9 (s, C-1"), 121.4 (d, C-11'), 124.0 (d, 2 C, C-2"), 124.3 (s, 2 C, C-3"), 125.8 (d, C-8'), $126.6\left(\mathrm{q},{ }^{1} J_{C, F}=211 \mathrm{~Hz}, 2 \mathrm{C}, \mathrm{CF}_{3}\right.$ ), $126.8\left(\mathrm{~d}, \mathrm{C}-7^{\prime *}\right), 127.4\left(\mathrm{~s}, \mathrm{C}-4 \mathrm{a}^{\prime}\right), 128.8\left(\mathrm{~d}, \mathrm{C}-9^{\prime}\right), 130.4\left(\mathrm{~s}, \mathrm{C}-9 \mathrm{a}^{\prime}\right), 132.7$ (d, C-6'*), 136.7 (s, C-5a'), 139.0 (s, C-10a'), 141.0 (s, C-3a'), 153.4 (s, C-11a'), 170.3 (s, C-2'), 182.9 (s, C-10'), 186.6 (s, NCSN).

MS (EI, $70 \mathrm{eV}): m / z(\%)=633\left(1,[\mathrm{M}]^{+}\right), 362(12), 345\left(39,\left[\mathrm{C}_{21} \mathrm{H}_{15} \mathrm{NO}_{2} \mathrm{~S}\right]^{+}\right)$, $317\left(52,\left[\mathrm{C}_{19} \mathrm{H}_{11} \mathrm{NO}_{2} \mathrm{~S}\right]^{+}\right), 271\left(100,\left[\mathrm{C}_{9} \mathrm{H}_{4} \mathrm{~F}_{6} \mathrm{NS}\right]^{+}\right), 229(18), 213(24)$, $163(13)$.

HRMS (ESI): $m / z[\mathrm{M}+\mathrm{H}]^{+}$calcd for $\mathrm{C}_{30} \mathrm{H}_{22} \mathrm{~F}_{6} \mathrm{~N}_{3} \mathrm{O}_{2} \mathrm{~S}_{2}: 634.1052$; found: 634.1047.

\section{(1R,2S)-2-Aminocyclohexane-1-carboxylic Acid Hydrochloride (21)}

Amino acid 20 ( $3.00 \mathrm{~g}, 12.1 \mathrm{mmol}, 1.00$ equiv) was dissolved in aq $6 \mathrm{~N}$ $\mathrm{HCl}(150 \mathrm{~mL})$. The mixture was stirred at $120^{\circ} \mathrm{C}$ for $48 \mathrm{~h}$. After this time, the solvent was evaporated and the product was obtained as a colorless solid (2.15 g, $12.0 \mathrm{mmol}, 99 \%)$; $\mathrm{mp} 198-220^{\circ} \mathrm{C}$.

${ }^{1} \mathrm{H}$ NMR (400 MHz, DMSO- $d_{6}$ ): $\delta=1.11-2.06$ (m, $8 \mathrm{H}, \mathrm{H}-3, \mathrm{H}-4, \mathrm{H}-5$, H-6), 2.89 (virt. q, $\left.{ }^{3} J \cong 3 J=4.4 \mathrm{~Hz}, 1 \mathrm{H}, \mathrm{H}-1\right), 3.08-3.41(\mathrm{~m}, 1 \mathrm{H}, \mathrm{H}-2)$, $8.06\left(\mathrm{~s}, 3 \mathrm{H}, \mathrm{NH}_{3}\right), 12.90(\mathrm{~s}, 1 \mathrm{H}, \mathrm{COOH})$.

${ }^{13} \mathrm{C}$ NMR (101 MHz, DMSO- $\left.d_{6}\right): \delta=22.0(\mathrm{t}, \mathrm{C}-4), 22.2(\mathrm{t}, \mathrm{C}-5), 25.5(\mathrm{t}$, C-6), 27.0 (t, C-3), 41.8 (d, C-1), 48.8 (d, C-2), 174.0 (s, COOH).

The analytical data are in accordance with the literature. ${ }^{27}$ 


\section{(1R,2S)-2-(1',3'-Dioxoisoindolin-2'-yl)cyclohexane-1-carboxylic Acid (22)}

Hydrochloride 21 (493 mg, $2.75 \mathrm{mmol}, 1.00$ equiv) was dissolved in anhyd toluene $(50 \mathrm{~mL})$. Subsequently phthalic anhydride $(427 \mathrm{mg}$, $2.88 \mathrm{mmol}, 1.05$ equiv) and $\mathrm{NEt}_{3}(556 \mathrm{mg}, 0.76 \mathrm{~mL}, 5.49 \mathrm{mmol}$, 2.00 equiv) were added. The mixture was stirred at $100^{\circ} \mathrm{C}$. After $18 \mathrm{~h}$, the solution was cooled and washed with aq $3 \mathrm{M} \mathrm{HCl}(150 \mathrm{~mL})$. The aqueous layer was extracted with $\mathrm{CH}_{2} \mathrm{Cl}_{2}(3 \times 150 \mathrm{~mL})$. The combined organic layers were dried $\left(\mathrm{Na}_{2} \mathrm{SO}_{4}\right)$ and filtered. The solvent was removed under reduced pressure. The desired product was obtained as a colorless solid (743 mg, $2.72 \mathrm{mmol}, 99 \%) ; \mathrm{mp} 144-146{ }^{\circ} \mathrm{C} ;[\alpha]_{\mathrm{D}}{ }^{20}$ $-52\left(c=1.00, \mathrm{CH}_{2} \mathrm{Cl}_{2}\right)$.

IR (ATR): 3285 (m, OH), $2960\left(\mathrm{~m}, \mathrm{CH}_{\mathrm{sp} 3}\right), 2925\left(\mathrm{w}, \mathrm{CH}_{\mathrm{sp} 3}\right), 2868(\mathrm{~m}$, $\left.\mathrm{CH}_{\mathrm{sp} 3}\right), 1719$ (s, C=O), 1690 (s, C=O), 1405 (m), $1372(\mathrm{~s}, \mathrm{C}-\mathrm{N}), 1331$ $(\mathrm{m}), 1184(\mathrm{~m}), 1082(\mathrm{~m}), 1018(\mathrm{~m}), 713 \mathrm{~cm}^{-1}\left(\mathrm{~m}, \mathrm{CH}_{\mathrm{sp} 2}\right)$.

${ }^{1} \mathrm{H}$ NMR $\left(400 \mathrm{MHz}, \mathrm{CDCl}_{3}\right): \delta=1.33-1.44(\mathrm{~m}, 1 \mathrm{H}, \mathrm{H}-5), 1.55$ (virt. dt, ${ }^{3} J=13.8 \mathrm{~Hz},{ }^{3} J \cong{ }^{3} J=4.0 \mathrm{~Hz}, 1 \mathrm{H}, \mathrm{H}-6$ ), 1.66 (virt. ddt, ${ }^{3} J=13.8 \mathrm{~Hz}$, $\left.12.4 \mathrm{~Hz},{ }^{3} \mathrm{~J} \cong 3 \mathrm{~J}=4.6 \mathrm{~Hz}, 1 \mathrm{H}, \mathrm{H}-5\right), 1.77\left(\mathrm{dd},{ }^{3} \mathrm{~J}=13.2 \mathrm{~Hz} .3 .6 \mathrm{~Hz}, 1 \mathrm{H}, \mathrm{H}-\right.$ 4), $1.90-2.01(\mathrm{~m}, 2 \mathrm{H}, \mathrm{H}-6, \mathrm{H}-3), 2.15$ (virt. dt, ${ }^{3} \mathrm{~J}=13.8 \mathrm{~Hz},{ }^{3} \cong$ ${ }^{3} J=2.8 \mathrm{~Hz}, 1 \mathrm{H}, \mathrm{H}-4$ ), 2.82 (virt. dq, ${ }^{3} J=12.7 \mathrm{~Hz},{ }^{3} \cong \cong{ }^{3} \mathrm{~J}=3.4 \mathrm{~Hz}, 1 \mathrm{H}, \mathrm{H}-$ 3), 3.00 (virt. q, ${ }^{3} \cong \cong{ }^{3} \mathrm{~J}=2.9 \mathrm{~Hz}, 1 \mathrm{H}, \mathrm{H}-1$ ), 4.33 (ddd, ${ }^{3} \mathrm{~J}=12.7 \mathrm{~Hz}$, $5.3 \mathrm{~Hz}, 3.5 \mathrm{~Hz}, 1 \mathrm{H}, \mathrm{H}-2), 7.66\left(\mathrm{dd},{ }^{3} J=5.5 \mathrm{~Hz},{ }^{4} J=3.0 \mathrm{~Hz}, 2 \mathrm{H}, \mathrm{H}-5^{\prime}, \mathrm{H}-\right.$ $6^{\prime}$ ), 7.77 (dd, ${ }^{3} \mathrm{~J}=5.5 \mathrm{~Hz},{ }^{4} \mathrm{~J}=3.0 \mathrm{~Hz}, 2 \mathrm{H}, \mathrm{H}-4^{\prime}, \mathrm{H}-7^{\prime}$ ), 8.42 (br s, $1 \mathrm{H}$, $\mathrm{COOH})$.

${ }^{13} \mathrm{C} \mathrm{NMR}\left(101 \mathrm{MHz}, \mathrm{CDCl}_{3}\right): \delta=21.6(\mathrm{t}, \mathrm{C}-5), 26.0(\mathrm{t}, \mathrm{C}-6), 26.1(\mathrm{t}, \mathrm{C}-4)$, 27.5 (t, C-3), 42.9 (d, C-1), 52.8 (d, C-2), 123.8 (d, 2 C, C-4', C-7'), 132.0 (s, 2 C, C-3a', C-7a'), 134.0 (d, 2 C, C-5', C-6'), 168.7 (s, 2 C, C-1', C-3'), $178.0(\mathrm{~s}, \mathrm{COOH})$.

MS (EI, $70 \mathrm{eV}): m / z(\%)=273\left(12,[\mathrm{M}]^{+}\right), 256\left(25,\left[\mathrm{C}_{15} \mathrm{H}_{13} \mathrm{NO}_{3}\right]^{+}\right), 227$ (15), 186 (23), $148\left(33,\left[\mathrm{C}_{8} \mathrm{H}_{6} \mathrm{NO}_{2}\right]^{+}\right), 91$ (100).

HRMS (EI): $m / z$ [M] $]^{+}$calcd for $\mathrm{C}_{15} \mathrm{H}_{15} \mathrm{NO}_{4}$ : 273.1001; found: 273.0996 .

\section{2'-Nitro-9'-oxo-9'H-thioxanthen-3'-yl (1R,2S)-2-(1",3"-Dioxoisoin-} dolin-2"-yl)cyclohexane-1-carboxylate (23)

The protected amino acid 22 (450 mg, $1.65 \mathrm{mmol}, 1.10$ equiv) was dissolved in anhyd $\mathrm{CH}_{2} \mathrm{Cl}_{2}(20 \mathrm{~mL})$ and oxalyl chloride $(209 \mathrm{mg}$, $0.14 \mathrm{~mL}, 1.65 \mathrm{mmol}, 1.10$ equiv) and a catalytic amount of DMF (5 drops) was added at rt. The mixture was stirred at $\mathrm{rt}$ for $3 \mathrm{~h}$. Meanwhile thioxanthone $\mathbf{9}$ ( $388 \mathrm{mg}, 1.50 \mathrm{mmol}, 1.00$ equiv) and a catalytic amount of 4-dimethylaminopyridine ( 5 crystals) was dissolved in anhyd $\mathrm{CH}_{2} \mathrm{Cl}_{2}(45 \mathrm{~mL})$ and cooled to $0{ }^{\circ} \mathrm{C}$. At this temperature, $\mathrm{NEt}_{3}$ ( $456 \mathrm{mg}, 0.63 \mathrm{~mL}, 4.50 \mathrm{mmol}, 3.00$ equiv) was added. At $0{ }^{\circ} \mathrm{C}$ the readily prepared solution of acid chloride was added slowly. The mixture was warmed to $\mathrm{rt}$ and stirred overnight. After $18 \mathrm{~h}$, aq $\mathrm{NH}_{4} \mathrm{Cl}$ $(80 \mathrm{~mL})$ was added and the layers were separated. The aqueous layer was extracted with $\mathrm{CH}_{2} \mathrm{Cl}_{2}(3 \times 100 \mathrm{~mL})$ and the combined organic layers were washed with aq $2 \mathrm{M} \mathrm{NaOH}(150 \mathrm{~mL})$, dried $\left(\mathrm{Na}_{2} \mathrm{SO}_{4}\right)$, and filtered. The solvent was evaporated and the desired product was obtained without further purification as a yellowish solid $(768 \mathrm{mg}$, $1.46 \mathrm{mmol}, 97 \%) ; \mathrm{mp} 188-190{ }^{\circ} \mathrm{C} ; R_{f}=0.86\left(\mathrm{CH}_{2} \mathrm{Cl}_{2} / \mathrm{MeOH} 98: 2\right)$ [UV, $\left.\mathrm{KMnO}_{4}\right] ;[\alpha]_{\mathrm{D}}{ }^{20}-216\left(c=1.00, \mathrm{CH}_{2} \mathrm{Cl}_{2}\right)$.

IR (ATR): $2948\left(\mathrm{w}, \mathrm{CH}_{\mathrm{sp} 3}\right), 2887\left(\mathrm{w}, \mathrm{CH}_{\mathrm{sp} 3}\right), 2847\left(\mathrm{w}, \mathrm{CH}_{\mathrm{sp} 3}\right), 1779(\mathrm{~s}$, $\mathrm{C}=\mathrm{O}), 1709(\mathrm{~s}, \mathrm{C}=\mathrm{O}), 1644(\mathrm{~s}, \mathrm{C}=\mathrm{O}), 1592\left(\mathrm{~s}, \mathrm{CH}_{\mathrm{sp} 2}\right), 1522\left(\mathrm{~s}, \mathrm{NO}_{2}\right), 1340$ $\left(\mathrm{s}, \mathrm{NO}_{2}\right), 1077$ (s), 1049 (s), $1017(\mathrm{~s}), 743\left(\mathrm{~s}, \mathrm{CH}_{\mathrm{sp} 2}\right), 723\left(\mathrm{~s}, \mathrm{CH}_{\mathrm{sp} 2}\right), 693$ $\mathrm{cm}^{-1}(\mathrm{C}-\mathrm{S}-\mathrm{C})$.

${ }^{1} \mathrm{H} \mathrm{NMR}\left(400 \mathrm{MHz}, \mathrm{CDCl}_{3}\right): \delta=1.50$ (virt. dt, ${ }^{3} \cong \cong 3 \mathrm{~J}=13.3 \mathrm{~Hz}, 3.9 \mathrm{~Hz}, 1$ H, H-5), 1.66-1.70 (m, 1 H, H-4), 1.79-1.89 (m, 2 H, H-3, H-6), 1.972.06 (m, 2 H, H-4, H-5), 2.40 (d, $3^{3}=14.7$ Hz, 1 H, H-6), 2.91 (virt. dq,
${ }^{3} J=12.8 \mathrm{~Hz} .{ }^{3} \mathrm{~J} \cong J=3.2 \mathrm{~Hz}, 1 \mathrm{H}, \mathrm{H}-3$ ), 3.38 (virt. q, ${ }^{3} J \cong{ }^{3} J=4.2 \mathrm{~Hz}, 1 \mathrm{H}$, $\mathrm{H}-1$ ), 4.48 (ddd, ${ }^{3} J=12.8 \mathrm{~Hz}, 5.1 \mathrm{~Hz}, 3.5 \mathrm{~Hz}, 1 \mathrm{H}, \mathrm{H}-2$ ), 7.57 (ddd, $\left.{ }^{3} J=8.2 \mathrm{~Hz} .7 .1 \mathrm{~Hz},{ }^{4} J=1.2 \mathrm{~Hz}, 1 \mathrm{H}, \mathrm{H}-7^{\prime}\right), 7.60-7.63$ (m, $2 \mathrm{H}, \mathrm{H}-4^{\prime}, \mathrm{H}-$ 5'), 7.69-7.74 (m, 3 H, H-6', H-5", H-6"), 7.86 (dd, ${ }^{3} J=5.4 \mathrm{~Hz}$, $\left.{ }^{4} J=3.0 \mathrm{~Hz}, 2 \mathrm{H}, \mathrm{H}-4^{\prime \prime}, \mathrm{H}^{\prime} 7^{\prime \prime}\right), 8.60$ (dd, ${ }^{3} J=8.2 \mathrm{~Hz},{ }^{4} J=1.5 \mathrm{~Hz}, 1 \mathrm{H}, \mathrm{H}-8^{\prime}$ ), 9.24 (s, $\left.1 \mathrm{H}, \mathrm{H}-1^{\prime}\right)$.

${ }^{13} \mathrm{C}$ NMR $\left(101 \mathrm{MHz}, \mathrm{CDCl}_{3}\right): \delta=21.0(\mathrm{t}, \mathrm{C}-4), 26.0(\mathrm{t}, \mathrm{C}-5), 26.2(\mathrm{t}, \mathrm{C}-3)$, 27.9 (t, C-6), 44.0 (d, C-1), 52.7 (d, C-2), 122.7 (d, C-4'), 123.4 (d, 2 C, C-4", C-7"), 126.3 (d, C-5'), 127.0 (s, C-9'), 127.7 (d, C-7'), 128.3 (d, C1'), 128.6 (s, C-8a'), 130.3 (d, C-8'), 132.0 (s, 2 C, C-3a", C-7a"), 133.4 (d, C-6'), 134.4, (d, 2 C, C-5", C-6"') 136.0 (s, C-4b'), 140.2 (s, C-3'), 144.2 (s, C-4a'), 146.2 (s, C-2'), 168.8 (s, 2 C, C-1", C-3"), 170.3 (s, COO), 178.0 (s, C-9').

MS (EI, $70 \mathrm{eV}): m / z(\%)=528\left(1,[\mathrm{M}]^{+}\right), 273\left(32,\left[\mathrm{C}_{13} \mathrm{H}_{7} \mathrm{NO}_{4} \mathrm{~S}\right]^{+}\right), 256$ $\left(100,\left[\mathrm{C}_{15} \mathrm{H}_{14} \mathrm{NO}_{3}\right]^{+}\right), 148\left(75,\left[\mathrm{C}_{8} \mathrm{H}_{6} \mathrm{NO}_{2}\right]^{+}\right)$.

HRMS (EI): $m / z[M]^{+}$calcd for $\mathrm{C}_{28} \mathrm{H}_{20} \mathrm{~N}_{2} \mathrm{O}_{7} \mathrm{~S}$ : 528.0991; found: 528.0992 .

(1R,2S)-2-(1',3'-Dioxoisoindolin-2'-yl)- $N$-(3"-hydroxy-9"-oxo-9"Hthioxanthen-2"-yl)cyclohexane-1-carboxamide (24)

Ester 23 (699 mg, $1.36 \mathrm{mmol}, 1.00$ equiv) was dissolved in THF $(40 \mathrm{~mL})$ and $\mathrm{H}_{2} \mathrm{O}(40 \mathrm{~mL})$ was added. To this solution In $(780 \mathrm{mg}$, $6.80 \mathrm{mmol}, 5.00$ equiv) and concd $\mathrm{HCl}(1.12 \mathrm{~mL})$ were added. The mixture was stirred at $80{ }^{\circ} \mathrm{C}$ and after $18 \mathrm{~h}$, the solution was cooled to $\mathrm{rt}$ and $\mathrm{CH}_{2} \mathrm{Cl}_{2}(50 \mathrm{~mL})$ was added. The mixture was filtered over Celite and washed with aq $\mathrm{NaHCO}_{3}(100 \mathrm{~mL})$. The aqueous layer was extracted with $\mathrm{CH}_{2} \mathrm{Cl}_{2}(3 \times 150 \mathrm{~mL})$. The combined organic layers were dried $\left(\mathrm{Na}_{2} \mathrm{SO}_{4}\right)$, filtered, and the solvent was removed under reduced pressure. Purification by flash chromatography $\left(4 \times 20 \mathrm{~cm}, \mathrm{CH}_{2} \mathrm{Cl}_{2}\right.$ / $\mathrm{MeOH} 95: 5)$ afforded the title product as an orange solid (597 mg, $1.24 \mathrm{mmol}, 91 \%) ; \mathrm{mp} 136-138{ }^{\circ} \mathrm{C} ; R_{f}=0.59\left(\mathrm{CH}_{2} \mathrm{Cl}_{2} / \mathrm{MeOH} 95: 5\right)$ [UV, $\left.\mathrm{KMnO}_{4}\right] ;[\alpha]_{\mathrm{D}}{ }^{20}-4\left(c=1.00, \mathrm{CH}_{2} \mathrm{Cl}_{2}\right)$.

IR (ATR): $3262(\mathrm{w}, \mathrm{OH}), 2933\left(\mathrm{w}, \mathrm{CH}_{\mathrm{sp} 3}\right), 2851\left(\mathrm{w}, \mathrm{CH}_{\mathrm{sp} 3}\right), 2552(\mathrm{w}$, CONH), 1769 (m, C=O), 1708 (s, C=O), $1658(\mathrm{~m}, \mathrm{CONH}), 1607$ (s, $\left.\mathrm{CH}_{\mathrm{sp} 2}\right), 1589\left(\mathrm{~s}, \mathrm{CH}_{\mathrm{sp} 2}\right), 1523(\mathrm{~s}, \mathrm{CONH}), 1490(\mathrm{~s}), 1437(\mathrm{~s}), 1077(\mathrm{~s})$, $1016(\mathrm{~s}), 742\left(\mathrm{CH}_{\mathrm{sp} 2}\right), 707 \mathrm{~cm}^{-1}\left(\mathrm{~s}, \mathrm{CH}_{\mathrm{sp} 2}\right)$.

${ }^{1} \mathrm{H}$ NMR $\left(400 \mathrm{MHz}, \mathrm{CDCl}_{3}\right): \delta=1.38-1.49(\mathrm{~m}, 1 \mathrm{H}, \mathrm{H}-4), 1.57-1.62(\mathrm{~m}$, $1 \mathrm{H}, \mathrm{H}-5$ ), 1.70 (virt. dt, ${ }^{3} \mathrm{~J}=13.3 \mathrm{~Hz},{ }^{3} \mathrm{~J} \cong{ }^{3} \mathrm{~J} \cong 4.2 \mathrm{~Hz}, 1 \mathrm{H}, \mathrm{H}-3$ ), 1.78 (virt. dt, $\left.{ }^{3} \mathrm{~J}=13.1 \mathrm{~Hz},{ }^{3} \mathrm{~J} \cong 3 \mathrm{~J} \cong 4.6 \mathrm{~Hz}, 1 \mathrm{H}, \mathrm{H}-6\right), 1.99-2.08(\mathrm{~m}, 1 \mathrm{H}, \mathrm{H}-$ 4), 2.08-2.22 (m, $1 \mathrm{H}, \mathrm{H}-5), 2.24-2.31$ ( $\mathrm{m}, 1 \mathrm{H}, \mathrm{H}-6), 2.97$ (virt. dq ${ }^{3} J=12.4 \mathrm{~Hz},{ }^{3} J \cong 3^{3}=3.7 \mathrm{~Hz}, 1 \mathrm{H}, \mathrm{H}-3$ ), 3.17 (virt. q, ${ }^{3} \mathrm{~J} \cong 3^{3} \mathrm{~J}=4.5 \mathrm{~Hz}, 1 \mathrm{H}$, $\mathrm{H}-1$ ), 4.35 (ddd, $\left.{ }^{3} \mathrm{~J}=12.4 \mathrm{~Hz}, 5.1 \mathrm{~Hz}, 3.8 \mathrm{~Hz}, 1 \mathrm{H}, \mathrm{H}-2\right), 7.15$ (s, $1 \mathrm{H}, \mathrm{H}-$ 4'), 7.48 (ddd, $\left.{ }^{3} \mathrm{~J}=8.2 \mathrm{~Hz}, 6.7 \mathrm{~Hz},{ }^{4} \mathrm{~J}=1.5 \mathrm{~Hz}, 1 \mathrm{H}, \mathrm{H}-7^{\prime}\right), 7.52-7.65(\mathrm{~m}$, 5 H, H-4", H-5", H-6", H-7", H-5'), 7.62 (ddd, 3J = 8.2 Hz, $6.7 \mathrm{~Hz}$, $\left.{ }^{4} J=1.5 \mathrm{~Hz}, 1 \mathrm{H}, \mathrm{H}-6^{\prime}\right), 7.88\left(\mathrm{~s}, 1 \mathrm{H}, \mathrm{H}-1^{\prime}\right), 8.42\left(\mathrm{dd},{ }^{3} \mathrm{~J}=8.2 \mathrm{~Hz}\right.$, $\left.{ }^{4} J=1.5 \mathrm{~Hz}, 1 \mathrm{H}, \mathrm{H}-8^{\prime}\right), 9.14(\mathrm{~s}, 1 \mathrm{H}, \mathrm{OH}), 10.65(\mathrm{~s}, 1 \mathrm{H}, \mathrm{NH})$.

${ }^{13} \mathrm{C} \mathrm{NMR}\left(101 \mathrm{MHz}, \mathrm{CDCl}_{3}\right): \delta=21.2(\mathrm{t}, \mathrm{C}-5), 25.8(\mathrm{t}, \mathrm{C}-4), 25.9(\mathrm{t}, \mathrm{C}-3)$, 28.9 (t, C-6), 45.0 (d, C-1), 52.6 (d, C-2), 115.4 (d, C-4'), 122.0 (s, C-4a'), 123.4 (d, 2 C, C-4", C-7"), 124.1 (d, C-1'), 126.2 (d, C-7'), 126.3 (d, C5'), 127.0 (s, C-3'), 128.7 (s, C-8a'), $129.4\left(\mathrm{~d}, \mathrm{C}-8^{\prime}\right), 131.4$ (s, 2 C, C-3a", C-7a"), 132.3 (d, C-6'), 134.2 (d, 2 C, C-5", C-6"), 137.0 (s, C-9a'), 137.7 (s, C-4b') 154.7 (s, C-2'), 168.6 (s, 2 C, C-1", C-3"), 175.3 (s, CONH), $179.5\left(\mathrm{~s}, \mathrm{C}-9^{\prime}\right)$

MS (EI, $70 \mathrm{eV}): m / z(\%)=498\left(5,[\mathrm{M}]^{+}\right), 256\left(30,\left[\mathrm{C}_{15} \mathrm{H}_{14} \mathrm{NO}_{3}\right]^{+}\right), 227(38$, $\left.\left[\mathrm{C}_{14} \mathrm{H}_{13} \mathrm{NO}_{2}\right]^{+}\right), 148\left(43,\left[\mathrm{C}_{8} \mathrm{H}_{4} \mathrm{NO}_{2}\right]^{+}\right), 111(55)$.

HRMS (ESI): $m / z$ [M $+\mathrm{H}]^{+}$calcd for $\mathrm{C}_{28} \mathrm{H}_{22} \mathrm{~N}_{2} \mathrm{O}_{5} \mathrm{~S}: 499.1322$; found: 499.1322 . 


\section{2-\{(1S,2R)-2-(10'-0xo-10'H-thioxantheno[2',3'-d]oxazol-2'-yl)cy-} clohexyl\}isoindoline-1",3"-dione (25)

The synthesized amide 24 (575 mg, $1.15 \mathrm{mmol}, 1.00$ equiv) was dissolved in anhyd THF $(55 \mathrm{~mL})$. At $\mathrm{rt}, \mathrm{PPh}_{3}(666 \mathrm{mg}, 2.54 \mathrm{mmol}$, 2.20 equiv) and diisopropyl azodicarboxylate $(513 \mathrm{mg}, 0.50 \mathrm{~mL}$, $2.54 \mathrm{mmol}, 2.20$ equiv) were added. The mixture was stirred at $\mathrm{rt}$ for $4 \mathrm{~h}$. After this time, the solvent was evaporated. The crude product was purified by flash chromatography $\left(4 \times 25 \mathrm{~cm}, \mathrm{CH}_{2} \mathrm{Cl}_{2} / \mathrm{MeOH}\right.$ 98:2). The desired product was obtained as a yellow solid $(510 \mathrm{mg}$, $1.06 \mathrm{mmol}, 89 \%)$; $\mathrm{mp} 95-98{ }^{\circ} \mathrm{C} ; R_{f}=0.64\left(\mathrm{CH}_{2} \mathrm{Cl}_{2} / \mathrm{MeOH} 98: 2\right)$ [UV, $\left.\mathrm{KMnO}_{4}\right] ;[\alpha]_{\mathrm{D}}{ }^{20}-176\left(c=1.00, \mathrm{CH}_{2} \mathrm{Cl}_{2}\right)$.

IR (ATR): 2932 (w, $\mathrm{CH}_{\mathrm{sp} 3}$ ), 2357 (w), 1708 (s, C=O), 1637 (m, C=N), $1619\left(\mathrm{~s}, \mathrm{C}=\mathrm{C}_{\mathrm{sp} 2}\right), 1436\left(\mathrm{~s}, \mathrm{CH}_{\mathrm{sp} 3}\right), 1366\left(\mathrm{~s}, \mathrm{CH}_{\mathrm{sp} 3}\right), 1292(\mathrm{~s}), 1108(\mathrm{~m})$, $740\left(\mathrm{~s}, \mathrm{CH}_{\mathrm{sp} 2}\right), 716\left(\mathrm{~s}, \mathrm{CH}_{\mathrm{sp} 2}\right), 698 \mathrm{~cm}^{-1}(\mathrm{~m}, \mathrm{C}-\mathrm{S}-\mathrm{C})$.

${ }^{1} \mathrm{H} \mathrm{NMR}\left(500 \mathrm{MHz}, \mathrm{CDCl}_{3}\right): \delta=1.56$ (virt. ddt, ${ }^{3} \mathrm{~J}=13.6 \mathrm{~Hz}, 9.6 \mathrm{~Hz},{ }^{3} \cong$ $\left.{ }^{3} \mathrm{~J}=4.7 \mathrm{~Hz}, 1 \mathrm{H}, \mathrm{H}-5\right), 1.71-1.80(\mathrm{~m}, 2 \mathrm{H}, \mathrm{H}-4, \mathrm{H}-6), 1.98-2.06(\mathrm{~m}, 1 \mathrm{H}$, H-3), 2.13-2.16 (m, $1 \mathrm{H}, \mathrm{H}-5$ ), 2.30-2.34 (m, $1 \mathrm{H}, \mathrm{H}-3$ ), 2.37 (virt. dt, $\left.{ }^{3} \mathrm{~J}=13.4 \mathrm{~Hz},{ }^{3} \mathrm{~J} \cong{ }^{3} \mathrm{~J}=4.1 \mathrm{~Hz}, 1 \mathrm{H}, \mathrm{H}-4\right), 2.88$ (virt. dq, ${ }^{3} \mathrm{~J}=13.2 \mathrm{~Hz},{ }^{3} \mathrm{~J} \cong$ $\left.{ }^{3} \mathrm{~J}=3.6 \mathrm{~Hz}, 1 \mathrm{H}, \mathrm{H}-6\right), 3.69-3.71(\mathrm{~m}, 1 \mathrm{H}, \mathrm{H}-2), 4.59$ (ddd, ${ }^{3} \mathrm{~J}=13.2 \mathrm{~Hz}$, $5.3 \mathrm{~Hz}, 3.6 \mathrm{~Hz}, 1 \mathrm{H}, \mathrm{H}-1$ ), 7.49 (ddd, ${ }^{3} \mathrm{~J}=8.1 \mathrm{~Hz}, 6.9 \mathrm{~Hz},{ }^{4} \mathrm{~J}=1.4 \mathrm{~Hz}, 1 \mathrm{H}$, H-8'), 7.57 (dd, $\left.{ }^{3} \mathrm{~J}=8.2 \mathrm{~Hz},{ }^{4} \mathrm{~J}=1.3 \mathrm{~Hz}, 1 \mathrm{H}, \mathrm{H}-6^{\prime}\right), 7.60$ (s, $\left.1 \mathrm{H}, \mathrm{H}-4^{\prime}\right)$, 7.63 (ddd, $\left.{ }^{3} \mathrm{~J}=8.2 \mathrm{~Hz}, 6.9 \mathrm{~Hz},{ }^{4} \mathrm{~J}=1.4 \mathrm{~Hz}, 1 \mathrm{H}, \mathrm{H}-7^{\prime}\right), 7.67-7.73(\mathrm{~m}, 4 \mathrm{H}$, H-4", H-5", H-6", H-7"), 8.63 (dd, ${ }^{3} J=8.1 \mathrm{~Hz},{ }^{4} J=1.4 \mathrm{~Hz}, 1 \mathrm{H}, \mathrm{H}-9^{\prime}$ ), 8.89 (s, $\left.1 \mathrm{H}, \mathrm{H}-11^{\prime}\right)$.

${ }^{13} \mathrm{C}$ NMR (101 MHz, $\left.\mathrm{CDCl}_{3}\right): \delta=21.3(\mathrm{t}, \mathrm{C}-5), 25.6(\mathrm{t}, \mathrm{C}-6), 26.4(\mathrm{t}, \mathrm{C}-4)$, 28.5 (t, C-3), 39.1 (d, C-2), 53.2 (d, C-1), 106.6 (d, C-4'), 122.0 (d, C11'), 123.4 (d, 2 C, C-4", C-7"), 125.7 (d, C-6'), 126.4 (d, C-8'), 126.7 (s, C-4a'), 128.9 (s, C-9a'), 130.1 (d, C-9'), 131.9 (s, 2 C, C-3a", C-7a"), 132.4 (d, C-7'), 134.2 (d, 2 C, C-5", C-6"), 137.2 (s, C-5a'), 137.8 (s, C10a'), 141.1 (s, C-3a'), 153.4 (s, C-11a'), 168.4 (s, 2 C, C-1", C-3"), 168.6 (s, C-2'), $180.0\left(\mathrm{~s}, \mathrm{C}-10^{\prime}\right)$.

MS (EI, $70 \mathrm{eV}): m / z(\%)=480\left(3,[\mathrm{M}]^{+}\right), 333\left(100,\left[\mathrm{C}_{20} \mathrm{H}_{15} \mathrm{NO}_{3} \mathrm{~S}\right]^{+}\right)$.

HRMS (ESI): $m / z[\mathrm{M}+\mathrm{H}]^{+}$calcd for $\mathrm{C}_{28} \mathrm{H}_{20} \mathrm{~N}_{2} \mathrm{O}_{4} \mathrm{~S}: 481.1217$; found: 481.1217.

\section{1-[3",5"-Bis(trifluoromethyl)phenyl]-3-\{(1S,2R)-2-(10'-oxo-10'H- thioxantheno[2',3'-d]oxazol-2'-yl)cyclohexyl\}thiourea (3)}

To a solution of oxazole $\mathbf{2 5}$ ( $510 \mathrm{mg}, 1.06 \mathrm{mmol}, 1.00$ equiv) in anhyd EtOH ( $35 \mathrm{~mL}$ ) were added anhyd $\mathrm{CH}_{2} \mathrm{Cl}_{2}(35 \mathrm{~mL})$ and ethylenediamine (638 mg, $0.71 \mathrm{~mL}, 10.6 \mathrm{mmol}, 10.0$ equiv) and the mixture was stirred at $50{ }^{\circ} \mathrm{C}$ for $24 \mathrm{~h}$. After this time, the solvent was evaporated, diluted with $\mathrm{CH}_{2} \mathrm{Cl}_{2}$, and filtered over $\mathrm{SiO}_{2}$. After evaporation of the solvent, the crude material was used in the next step without further purification. The crude material was dissolved in anhyd THF $(70 \mathrm{~mL})$ and 1-isothioxyanato-3,5-bis(trifluoromethyl)benzene (575 mg, $0.39 \mathrm{~mL}, 2.12 \mathrm{mmol}, 2.00$ equiv) was added. The reaction mixture was stirred at rt and after $18 \mathrm{~h}$, the solvent was evaporated. Purification by flash chromatography $(2 \times 15 \mathrm{~cm}, \mathrm{Pn} /$ EtOAc 4:1) afforded the final thiourea as a yellow solid ( $300 \mathrm{mg}, 0.48 \mathrm{mmol}, 46 \%$ over 2 steps); $\operatorname{mp~} 126-128^{\circ} \mathrm{C} ; R_{f}=0.22(\mathrm{Pn} / \mathrm{EtOAc} 4: 1)$ [UV, $\left.\mathrm{KMnO}_{4}\right] ;[\alpha]_{\mathrm{D}}{ }^{20}-216$ (c $=1.00, \mathrm{CH}_{2} \mathrm{Cl}_{2}$ ).

IR (ATR): 2936 (w, $\left.\mathrm{CH}_{\mathrm{sp} 3}\right), 1711$ (s, C=0), 1607 (m, C=C $\left.\mathrm{sp2}\right), 1589$ (m, $\left.\mathrm{C}=\mathrm{C}_{\mathrm{sp} 2}\right), 1520\left(\mathrm{~s}, \mathrm{C}=\mathrm{C}_{\mathrm{sp} 2}\right), 1436\left(\mathrm{~s}, \mathrm{CH}_{\mathrm{p} 3}\right), 1383(\mathrm{~s}), 1274(\mathrm{~s}), 1174(\mathrm{~s})$, $1129(\mathrm{~s}), 743\left(\mathrm{~s}, \mathrm{CH}_{\mathrm{p} 3}\right), 698 \mathrm{~cm}^{-1}(\mathrm{~m}, \mathrm{C}-\mathrm{S}-\mathrm{C})$.

${ }^{1} \mathrm{H}$ NMR $\left(400 \mathrm{MHz}\right.$, DMSO- $\left.d_{6}\right): \delta=1.51-1.89(\mathrm{~m}, 6 \mathrm{H}, \mathrm{H}-3, \mathrm{H}-4, \mathrm{H}-5$, $\mathrm{H}-6), 1.99-2.15$ (m, $2 \mathrm{H}, \mathrm{H}-3, \mathrm{H}-6), 3.79$ (virt. td, ${ }^{3} \mathrm{~J} \cong{ }^{3} \mathrm{~J}=7.4 \mathrm{~Hz}$, ${ }^{3} \mathrm{~J}=4.3 \mathrm{~Hz}, 1 \mathrm{H}, \mathrm{H}-2$ ), 4.99 (virt. tt, ${ }^{3} \cong \cong{ }^{3} \mathrm{~J}=8.3 \mathrm{~Hz}, 4.0 \mathrm{~Hz}, 1 \mathrm{H}, \mathrm{H}-1$ ), $7.58\left(\mathrm{ddd},{ }^{3} \mathrm{~J}=8.2,6.8 \mathrm{~Hz},{ }^{4} \mathrm{~J}=1.5 \mathrm{~Hz}, 1 \mathrm{H}, \mathrm{H}-8^{\prime}\right), 7.62\left(\mathrm{~s}, 1 \mathrm{H}, \mathrm{H}-4^{\prime}\right)$,
7.70-7.84 (m, 2 H, H-6', H-7'), 8.07 (d, $\left.{ }^{3} J=8.6 \mathrm{~Hz}, 1 \mathrm{H}, \mathrm{NH}\right), 8.16$ (s, 1 H, H-4"), 8.20 (s, $\left.2 \mathrm{H}, \mathrm{H}-2^{\prime \prime}\right), 8.49$ (dd, ${ }^{3} J=8.2 \mathrm{~Hz},{ }^{4} \mathrm{~J}=1.4 \mathrm{~Hz}, 1 \mathrm{H}, \mathrm{H}-9^{\prime}$ ), $8.73\left(\mathrm{~s}, 1 \mathrm{H}, \mathrm{H}-11^{\prime}\right), 9.97(\mathrm{~s}, 1 \mathrm{H}, \mathrm{NH})$.

${ }^{13} \mathrm{C}$ NMR (101 MHz, DMSO- $\left.d_{6}\right): \delta=22.1\left(\mathrm{t}, \mathrm{C}-4^{*}\right), 22.4\left(\mathrm{t}, \mathrm{C}-5^{*}\right), 25.9(\mathrm{t}$, C-3), 28.3 (t, C-6), 38.8 (d, C-2), 52.0 (d, C-1), 107.7 (d, C-4"), 116.1 (d, C-4'), 116.2 (s, C-1"), 120.0 (d, C-11') 120.9 (q, $\left.{ }^{1} J_{C, F}=183 \mathrm{~Hz}, 2 \mathrm{C}, \mathrm{CF}_{3}\right)$, 121.8 (d, 2 C, C-2"), 126.1 (q, $\left.{ }^{2} J_{\mathrm{C}, \mathrm{F}}=109 \mathrm{~Hz}, 2 \mathrm{C}, \mathrm{C}-3^{\prime \prime}\right), 126.2\left(\mathrm{~d}, \mathrm{C}-\mathrm{7}^{* *}\right)$, 126.7 (d, C-8'), 127.7 (s, C-4a'), 129.1 (d, C-9'), 132.9 (d, C-6'*), 133.5 (s, C-9a'), 136.5 (s, C-5a'), 140.7 (s, C-10a'), 141.6 (s, C-3a'), 152.6 (s, C-11a'), 169.1 (s, C-2'), 178.6 (s, NCSN), 179.9 (s, C-10').

MS (EI, $70 \mathrm{eV}): m / z(\%)=621\left(1,[\mathrm{M}]^{+}\right), 578\left(2,\left[\mathrm{C}_{29} \mathrm{H}_{19} \mathrm{~F}_{6} \mathrm{~N}_{3} \mathrm{O}_{2} \mathrm{~S}\right]^{+}\right), 392$ $\left(15, \quad\left[\mathrm{C}_{16} \mathrm{H}_{12} \mathrm{~F}_{6} \mathrm{~N}_{3} \mathrm{~S}\right]^{+}\right), 333 \quad\left(63, \quad\left[\mathrm{C}_{10} \mathrm{H}_{18} \mathrm{~F}_{3} \mathrm{~N}_{3} \mathrm{O}_{2} \mathrm{~S}_{2}\right]^{+}\right), \quad 271 \quad(100$, $\left.\left[\mathrm{C}_{9} \mathrm{H}_{4} \mathrm{~F}_{6} \mathrm{NS}\right]^{+}\right), 229\left(67,\left[\mathrm{C}_{8} \mathrm{H}_{5} \mathrm{~F}_{6} \mathrm{~N}\right]^{+}\right)$.

HRMS (ESI): $m / z$ [M + H] ${ }^{+}$calcd for $\mathrm{C}_{29} \mathrm{H}_{21} \mathrm{~F}_{6} \mathrm{~N}_{3} \mathrm{O}_{2} \mathrm{~S}_{2}: 622.1052$; found: 622.1045 .

\section{tert-Butyl [(1S,2R)-2-(Hydroxymethyl)cyclohexyl]carbamate (27)}

To a solution of $[(1 S, 2 R)-2$-aminocyclohexyl]methanol $(\mathbf{2 6} ; 130 \mathrm{mg}$, $1.01 \mathrm{mmol}, 1.00$ equiv) in $\mathrm{CH}_{2} \mathrm{Cl}_{2}(2 \mathrm{~mL})$ was added $\mathrm{Et}_{3} \mathrm{~N}(280 \mu \mathrm{L}$, $203 \mathrm{mg}, 2.01 \mathrm{mmol}, 2.00$ equiv). The solution was cooled to $0{ }^{\circ} \mathrm{C}$ and $\mathrm{Boc}_{2} \mathrm{O}$ (230 mg, $1.06 \mathrm{mmol}, 1.05$ equiv) was added. The solution was stirred and allowed to warm to $\mathrm{rt}$ overnight. The reaction was quenched by the addition of aq $1 \mathrm{M} \mathrm{HCl}$. The layers were separated and the aqueous layer was extracted with $\mathrm{CH}_{2} \mathrm{Cl}_{2}(2 \times 5 \mathrm{~mL})$. The organic layers were combined and successively washed with aq $\mathrm{NaHCO}_{3}$ $(2 \times 15 \mathrm{~mL})$ and brine $(2 \times 15 \mathrm{~mL})$, and dried $\left(\mathrm{Na}_{2} \mathrm{SO}_{4}\right)$. The solvent was removed under reduced pressure and the residue was purified by flash chromatography $(3 \times 10 \mathrm{~cm}$, Chx/EtOAc 9:1 $\rightarrow$ 4:1). Product 27 was obtained as a yellow oil (213 $\mathrm{mg}, 928 \mu \mathrm{mol}, 92 \%) ; R_{f}=0.17$ (Chx/EtOAc 4:1) [UV, KMnO4]; $[\alpha]_{D}^{20}-30\left(c=1.00, \mathrm{CH}_{2} \mathrm{Cl}_{2}\right)$.

${ }^{1} \mathrm{H}$ NMR $\left(500 \mathrm{MHz}, \mathrm{CDCl}_{3}\right): \delta=0.86-0.98(\mathrm{~m}, 1 \mathrm{H}, \mathrm{H}-5), 1.14-1.36(\mathrm{~m}$, $3 \mathrm{H}, \mathrm{H}-3, \mathrm{H}-4, \mathrm{H}-6), 1.45$ [s, $\left.9 \mathrm{H}, \mathrm{C}\left(\mathrm{CH}_{3}\right)_{3}\right], 1.52-1.83$ (m, $5 \mathrm{H}, \mathrm{H}-2, \mathrm{H}-3$, $\mathrm{H}-5, \mathrm{H}-6, \mathrm{OH}$ ), 3.21 (virt. t, ${ }^{3} \cong{ }^{3} \mathrm{~J}=11.4 \mathrm{~Hz}, 1 \mathrm{H}, \mathrm{CHHOH}$ ), 3.34 (dd, ${ }^{2} \mathrm{~J}=$ $\left.11.9 \mathrm{~Hz},{ }^{3} \mathrm{~J}=4.7 \mathrm{~Hz}, 1 \mathrm{H}, \mathrm{CHHOH}\right), 4.04-4.07$ (m, $1 \mathrm{H}, \mathrm{H}-1$ ), 4.77 (d, ${ }^{3} \mathrm{~J}=$ $9.0 \mathrm{~Hz}, 1 \mathrm{H}, \mathrm{NH})$.

${ }^{13} \mathrm{C} \mathrm{NMR}\left(126 \mathrm{MHz}, \mathrm{CDCl}_{3}\right): \delta=21.0(\mathrm{t}, \mathrm{C}-5), 23.1(\mathrm{t}, \mathrm{C}-4), 24.9(\mathrm{t}, \mathrm{C}-3)$, $28.2\left(\mathrm{q}, \mathrm{CH}_{3}\right), 30.3(\mathrm{t}, \mathrm{C}-6), 43.1$ (d, C-2), 45.1 (d, C-1), $63.9\left(\mathrm{t}, \mathrm{CH}_{2} \mathrm{OH}\right)$, $80.0\left[\mathrm{~s}, \mathrm{C}\left(\mathrm{CH}_{3}\right)_{3}\right], 157.2$ (s, NHCO).

The spectroscopic data match the literature values. ${ }^{30 a}$

\section{tert-Butyl [(1S,2R)-2-Formylcyclohexyl]carbamate (28)}

A solution of oxalyl chloride $(0.55 \mathrm{~mL}, 824 \mathrm{mg}, 6.50 \mathrm{mmol}, 1.00$ equiv) in $\mathrm{CH}_{2} \mathrm{Cl}_{2}(16 \mathrm{~mL})$ was cooled to $-78^{\circ} \mathrm{C}$, before a solution of DMSO ( $1.38 \mathrm{~mL}, 1.52 \mathrm{~g}, 19.5 \mathrm{mmol}, 3.00$ equiv) in $\mathrm{CH}_{2} \mathrm{Cl}_{2}(2 \mathrm{~mL})$ was added dropwise. The solution was stirred for $1 \mathrm{~h}$ at $-78{ }^{\circ} \mathrm{C}$. Subsequently, a solution of carbamate 27 (1.49 g, $6.50 \mathrm{mmol}, 1.00$ equiv) in $\mathrm{CH}_{2} \mathrm{Cl}_{2}$ (7 $\mathrm{mL}$ ) was added slowly over $10 \mathrm{~min}$. After an additional $10 \mathrm{~min}, \mathrm{NEt}_{3}$ ( $4.50 \mathrm{~mL}, 3.30 \mathrm{~g}, 32.5 \mathrm{mmol}, 5.00$ equiv) was added. The reaction mixture was stirred for an additional $15 \mathrm{~min}$ at $-78^{\circ} \mathrm{C}$ and then allowed to warm to rt. The reaction was quenched by the addition of $\mathrm{H}_{2} \mathrm{O}(20$ $\mathrm{mL}$ ). The layers were separated and the aqueous layer was extracted with $\mathrm{CH}_{2} \mathrm{Cl}_{2}(2 \times 20 \mathrm{~mL})$. The combined organic layers were washed successively with aq $\mathrm{NH}_{4} \mathrm{Cl}(1 \times 60 \mathrm{~mL})$ and brine $(1 \times 60 \mathrm{~mL})$, dried $\left(\mathrm{Na}_{2} \mathrm{SO}_{4}\right)$, and filtered. All volatiles were removed under reduced pressure and the product $\mathbf{2 8}$ was obtained as a brownish solid (1.39 g, $6.12 \mathrm{mmol}, 94 \%)$ and used in the next step without further purification; $\mathrm{mp} 23-25^{\circ} \mathrm{C} ;[\alpha]_{\mathrm{D}}^{20}+82.4\left(c=0.17, \mathrm{CH}_{2} \mathrm{Cl}_{2}\right)$. 
${ }^{1} \mathrm{H}$ NMR (300 MHz, $\left.\mathrm{CDCl}_{3}\right): \delta=1.19-1.38(\mathrm{~m}, 1 \mathrm{H}, \mathrm{H}-5), 1.43[\mathrm{~s}, 9 \mathrm{H}$, $\left.\mathrm{C}\left(\mathrm{CH}_{3}\right)_{3}\right], 1.53-1.77$ ( $\left.\mathrm{m}, 6 \mathrm{H}, \mathrm{H}-3, \mathrm{H}-4, \mathrm{H}-5, \mathrm{H}-6\right), 1.91-2.01(\mathrm{~m}, 1 \mathrm{H}, \mathrm{H}-$ 3), 2.71 (virt. q, ${ }^{3} \cong{ }^{3} J=4.6 \mathrm{~Hz}, 1 \mathrm{H}, \mathrm{H}-2$ ), 3.97 (virt. tt, ${ }^{3} \cong 9.1,4.1 \mathrm{~Hz}$, $1 \mathrm{H}, \mathrm{H}-1$ ), 5.15-5.31 (m, $1 \mathrm{H}, \mathrm{NH}), 9.70$ (d, ${ }^{3} \mathrm{~J}=4.2 \mathrm{~Hz}, 1 \mathrm{H}, \mathrm{CHO}$ ).

${ }^{13} \mathrm{C}$ NMR $\left(126 \mathrm{MHz}, \mathrm{CDCl}_{3}\right): \delta=22.9(\mathrm{t}, \mathrm{C}-3), 23.7(\mathrm{t}, \mathrm{C}-5), 23.9(\mathrm{t}, \mathrm{C}-4)$, 28.5 (q, $\mathrm{CH}_{3}$ ), 29.8 (t, C-6), 48.2 (d, C-1), 52.1 (d, C-2), 79.6 [s, $\mathrm{C}\left(\mathrm{CH}_{3}\right)_{3}$ ], 155.5 (s, NHCO), 204.8 (s, CHO).

The spectroscopic data match the literature values. ${ }^{30 a}$

\section{tert-Butyl [(1S,2S)-2-Ethynylcyclohexyl]carbamate (29)}

A solution of dimethyl(diazomethyl)phosphonate $(1.90 \mathrm{~g}, 12.6 \mathrm{mmol}$, 2.05 equiv) in THF $(15 \mathrm{~mL})$ was cooled to $-78{ }^{\circ} \mathrm{C}$. KO $\mathrm{Bu}(1.45 \mathrm{~g}$, $12.9 \mathrm{mmol}, 2.10$ equiv) was added and the solution was stirred for 1 h. Subsequently, a solution of aldehyde 28 ( $1.39 \mathrm{~g}, 6.16 \mathrm{mmol}, 1.00$ equiv) in THF (190 mL) was added slowly. The reaction mixture was stirred for $15 \mathrm{~h}$ and was allowed to warm to $\mathrm{rt}$ during that time. The reaction was quenched by the addition of aq $\mathrm{NH}_{4} \mathrm{Cl}(80 \mathrm{~mL})$. The layers were separated and the aqueous layer was extracted with EtOAc $(3 \times 80 \mathrm{~mL})$. The combined organic layers were successively washed with aq $\mathrm{NH}_{4} \mathrm{Cl}(2 \times 250 \mathrm{~mL})$ and brine $(2 \times 250 \mathrm{~mL})$, dried $\left(\mathrm{Na}_{2} \mathrm{SO}_{4}\right)$, and all the volatiles were removed under reduced pressure. The crude product was purified by flash chromatography $(4 \times 20 \mathrm{~cm}$, Chx/EtOAc 10:1) to afford 29 as a colorless solid $(0.91 \mathrm{~g}, 4.07 \mathrm{mmol}$, $67 \%) ; \mathrm{mp} 50{ }^{\circ} \mathrm{C} ; R_{f}=0.64\left(\mathrm{Chx} /\right.$ EtOAc 4:1) [UV, $\left.\mathrm{KMnO}_{4}\right]$; $[\alpha]_{\mathrm{D}}{ }^{20}-24$ (c=1.00, $\mathrm{CH}_{2} \mathrm{Cl}_{2}$ ).

IR (ATR): 3437 (w, N-H), 3309 (w, C-H), 2977 (w, $\left.\mathrm{CH}_{3}\right), 2934\left(\mathrm{~m}, \mathrm{CH}_{2}\right)$, 2860 (w, C-H), 2111 (w, C=C), $1702(\mathrm{~s}, \mathrm{C}=\mathrm{O}), 1497(\mathrm{~s}, \mathrm{~N}-\mathrm{H}), 1365(\mathrm{~m})$, $1245(\mathrm{~m}), 1165(\mathrm{~s}), 944(\mathrm{w}), 864(\mathrm{w}), 779(\mathrm{w}), 626 \mathrm{~cm}^{-1}(\mathrm{~m})$.

${ }^{1} \mathrm{H}$ NMR (400 MHz, $\mathrm{CDCl}_{3}$ ): $\delta=1.22-1.34(\mathrm{~m}, 1 \mathrm{H}, \mathrm{H}-5), 1.44[\mathrm{~s}, 9 \mathrm{H}$, $\left.\mathrm{C}\left(\mathrm{CH}_{3}\right)_{3}\right], 1.48-1.66(\mathrm{~m}, 4 \mathrm{H}, \mathrm{H}-3, \mathrm{H}-4, \mathrm{H}-6), 1.67-1.76(\mathrm{~m}, 2 \mathrm{H}, \mathrm{H}-5, \mathrm{H}-$ 6), $1.80-1.89$ (m, $1 \mathrm{H}, \mathrm{H}-3$ ), 2.11 (d, ${ }^{4} \mathrm{~J}=2.5 \mathrm{~Hz}, 1 \mathrm{H}, \mathrm{C} \equiv \mathrm{CH}$ ), 2.96 (br s, 1 $\mathrm{H}, \mathrm{H}-2$ ), 3.56 (virt. ddt, ${ }^{3} \mathrm{~J}=13.1 \mathrm{~Hz}, 8.6 \mathrm{~Hz},{ }^{3} \mathrm{~J} \cong 3^{3} \mathrm{~J}=4.0 \mathrm{~Hz}, 1 \mathrm{H}, \mathrm{H}-1$ ), $4.80\left(\mathrm{~d},{ }^{3} \mathrm{~J}=9.4 \mathrm{~Hz}, 1 \mathrm{H}, \mathrm{NH}\right)$.

${ }^{13} \mathrm{C}$ NMR (126 MHz, $\left.\mathrm{CDCl}_{3}\right): \delta=20.8(\mathrm{t}, \mathrm{C}-4), 25.1(\mathrm{t}, \mathrm{C}-5), 28.6(\mathrm{q}$, $\mathrm{CH}_{3}$ ), 29.0 (t, C-6), 30.2 (t, C-3), 33.4 (d, C-2), 50.5 (d, C-1), 71.9 (d, $\mathrm{C} \equiv \mathrm{CH}), 79.4\left[\mathrm{~s}, \mathrm{C}\left(\mathrm{CH}_{3}\right)_{3}\right], 84.1(\mathrm{~s}, \mathrm{C} \equiv \mathrm{CH}), 155.2$ (s, NHCO).

MS (EI, $70 \mathrm{eV}): m / z(\%)=167\left(30,\left[\mathrm{C}_{9} \mathrm{H}_{13} \mathrm{NO}_{2}\right]^{+}\right), 123\left(27,\left[\mathrm{C}_{9} \mathrm{H}_{13} \mathrm{~N}\right]^{+}\right)$, $106\left(27,\left[\mathrm{C}_{8} \mathrm{H}_{10}\right]^{+}\right), 57\left(100,\left[\mathrm{C}_{4} \mathrm{H}_{8}\right]^{+}\right)$.

HRMS (ESI): $m / z[\mathrm{M}+\mathrm{H}]^{+}$calcd for $\mathrm{C}_{13} \mathrm{H}_{22} \mathrm{NO}_{2}$ : 224.1645; found: 224.1645 .

\section{tert-Butyl $\left\{(1 S, 2 S)-2-\left[\left(9^{\prime}-0 \times 0-9 ' H-\right.\right.\right.$ thioxanthen-2'-yl)-ethynyl]cy- clohexyl\}carbamate (31)}

Alkyne 29 ( $80 \mathrm{mg}, 360 \mu \mathrm{mol}, 1.00$ equiv) and bromothioxanthone $\mathbf{3 0}^{34}$ (114 mg, $394 \mu \mathrm{mol}, 1.10$ equiv) were dissolved in anhyd THF (18 $\mathrm{mL})$ and freshly distilled $\mathrm{NEt}_{3}(18 \mathrm{~mL})$. The solution was degassed three times by the freeze-pump-thaw ${ }^{37}$ method. Subsequently, $\mathrm{Pd}\left(\mathrm{PPh}_{3}\right)_{4}$ (41.4 mg, $35.8 \mu \mathrm{mol}, 0.10$ equiv) and $\mathrm{CuI}(13.6 \mathrm{mg}, 71.6$ $\mu \mathrm{mol}, 0.20$ equiv) were added and the mixture was again degassed four times by the freeze-pump-thaw method. The mixture was heated to $60{ }^{\circ} \mathrm{C}$ for $16 \mathrm{~h}$ in a sealed tube. After cooling to rt, the volatiles were removed under reduced pressure. The black residue was dissolved in $\mathrm{CH}_{2} \mathrm{Cl}_{2}(20 \mathrm{~mL})$ and the organic layer was washed successively with aq $\mathrm{NH}_{4} \mathrm{Cl}(2 \times 20 \mathrm{~mL})$ and brine $(2 \times 20 \mathrm{~mL})$. The organic layer was dried $\left(\mathrm{Na}_{2} \mathrm{SO}_{4}\right)$ and the solvent was removed under reduced pressure. The crude product was purified by flash chromatography
( $2 \times 20 \mathrm{~cm}, \mathrm{Chx} /$ EtOAc 50:1 $\rightarrow$ 20:1 $\rightarrow 5: 1$ ) to give the title compound as a bright yellow solid ( $121 \mathrm{mg}, 280 \mu \mathrm{mol}, 78 \%) ; \mathrm{mp} 185-187^{\circ} \mathrm{C} ; R_{f}=$ 0.16 (Chx/EtOAc 20:1) [UV, $\mathrm{KMnO}_{4}$ ]; $[\alpha]_{\mathrm{D}}{ }^{20}-154\left(c=1.00, \mathrm{CH}_{2} \mathrm{Cl}_{2}\right)$.

IR (ATR): 3364 (w, N-H), 3058 (w, ArH), 2973 (w, $\mathrm{CH}_{3}$ ), 2934 (w, $\mathrm{CH}_{2}$ ), 2859 (w, C-H), 2221 (w, C $\equiv$ C), 1681 ( $\mathrm{m}, \mathrm{C}=\mathrm{O}), 1642$ ( $\mathrm{m}, \mathrm{C}=\mathrm{O}), 1438$ ( $\mathrm{m}$, $\mathrm{N}-\mathrm{H}), 1248(\mathrm{~m}), 1162(\mathrm{~m}), 894(\mathrm{w}), 825(\mathrm{w}), 743(\mathrm{~m}), 620 \mathrm{~cm}^{-1}(\mathrm{w})$.

${ }^{1} \mathrm{H}$ NMR (400 MHz, CDCl $): \delta=1.32-1.40(\mathrm{~m}, 1 \mathrm{H}, \mathrm{H}-5), 1.47[\mathrm{~s}, 9 \mathrm{H}$, $\left.\mathrm{C}\left(\mathrm{CH}_{3}\right)_{3}\right], 1.57-1.71(\mathrm{~m}, 4 \mathrm{H}, \mathrm{H}-3, \mathrm{H}-4, \mathrm{H}-6), 1.75-1.81(\mathrm{~m}, 2 \mathrm{H}, \mathrm{H}-5, \mathrm{H}-$ 6), 1.93-2.02 (m, $1 \mathrm{H}, \mathrm{H}-3), 3.18-3.26(\mathrm{~m}, 1 \mathrm{H}, \mathrm{H}-2), 3.62-3.72(\mathrm{~m}, 1$ $\mathrm{H}, \mathrm{H}-1), 4.85\left(\mathrm{~d},{ }^{3} \mathrm{~J}=9.5 \mathrm{~Hz}, 1 \mathrm{H}, \mathrm{NH}\right), 7.53\left(\mathrm{dd},{ }^{3} \mathrm{~J}=7.2 \mathrm{~Hz},{ }^{4} \mathrm{~J}=1.4 \mathrm{~Hz}, 1\right.$ H, H-4'), 7.55 (d, $\left.{ }^{3} \mathrm{~J}=8.4 \mathrm{~Hz}, 1 \mathrm{H}, \mathrm{H}-7^{\prime}\right), 7.61\left(\mathrm{dd},{ }^{3} \mathrm{~J}=8.1 \mathrm{~Hz},{ }^{4} \mathrm{~J}=1.4 \mathrm{~Hz}\right.$ $1 \mathrm{H}, \mathrm{H}-5^{\prime}$ ), 7.64-7.66 (m, $\left.2 \mathrm{H}, \mathrm{H}-3^{\prime}, \mathrm{H}-6^{\prime}\right), 8.63$ (dd, ${ }^{3} \mathrm{~J}=8.1 \mathrm{~Hz},{ }^{4} \mathrm{~J}=1.5$ $\left.\mathrm{Hz}, 1 \mathrm{H}, \mathrm{H}-8^{\prime}\right), 8.66$ (d, $\left.{ }^{4} \mathrm{~J}=1.8 \mathrm{~Hz}, 1 \mathrm{H}, \mathrm{H}-1^{\prime}\right)$.

${ }^{13} \mathrm{C}$ NMR (101 MHz, $\left.\mathrm{CDCl}_{3}\right): \delta=21.2(\mathrm{t}, \mathrm{C}-4), 25.2(\mathrm{t}, \mathrm{C}-5), 28.6(\mathrm{q}$, $\mathrm{CH}_{3}$ ), 29.5 (t, C-6), 30.6 (t, C-3), 34.4 (d, C-2), 51.0 (d, C-1), 79.6 [s,

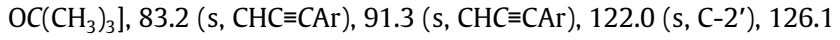
(d, C-5'), 126.2 (d, C-4'), 126.7 (d, C-7'), 128.8 (s, C-8a'), 129.2 (s, C$\left.1 \mathrm{a}^{\prime}\right), 130.1$ (d, C-8'), 132.6 (d, C-6'), $133.1\left(\mathrm{~d}, \mathrm{C}-1^{\prime}\right), 135.1$ (d, C-3'), 136.8 (s, C-4a'), 137.1 (s, C-5a'), 155.3 (s, NHCO), 179.5 (s, C=0).

MS (EI, $70 \mathrm{eV}): m / z(\%)=333\left(100,\left[\mathrm{M}-\mathrm{CO}_{2} t-\mathrm{Bu}\right]^{+}\right), 290(89$, $\left.\left[\mathrm{C}_{19} \mathrm{H}_{14} \mathrm{OS}\right]^{+}\right), 237\left(92,\left[\mathrm{C}_{15} \mathrm{H}_{9} \mathrm{OS}\right]^{+}\right), 139\left(11,\left[\mathrm{C}_{7} \mathrm{H}_{7} \mathrm{OS}\right]^{+}\right)$.

HRMS (ESI): $m / z[\mathrm{M}+\mathrm{H}]^{+}$calcd for $\mathrm{C}_{26} \mathrm{H}_{28} \mathrm{NO}_{3} \mathrm{~S}$ : 434.1784; found: 434.1784 .

\section{2'-\{[(1S,2S)-2-Aminocyclohexyl]ethynyl\}-9' H-thioxanthen-9'-one (32)}

Boc-protected amine $\mathbf{3 1}$ (75 mg, $173 \mu \mathrm{mol}, 1.00$ equiv) was dissolved in $\mathrm{CH}_{2} \mathrm{Cl}_{2}(2 \mathrm{~mL})$ and cooled to $0{ }^{\circ} \mathrm{C}$. TFA $(132 \mu \mathrm{L}, 197 \mathrm{mg}, 1.73 \mathrm{mmol}$, 10.0 equiv) was slowly added and the solution was stirred at rt for $2 \mathrm{~h}$. The reaction was quenched by the addition of $\mathrm{H}_{2} \mathrm{O}(5 \mathrm{~mL})$ and the layers were separated. The aqueous layer was extracted with $\mathrm{CH}_{2} \mathrm{Cl}_{2}(2 \times$ $5 \mathrm{~mL}$ ). The combined organic layers were successively washed with aq $\mathrm{NaHCO}_{3}(2 \times 15 \mathrm{~mL})$ and brine $(1 \times 15 \mathrm{~mL})$, dried $\left(\mathrm{Na}_{2} \mathrm{SO}_{4}\right)$, and the volatiles were removed under reduced pressure. The crude product was purified by flash column chromatography $\left(2 \times 20 \mathrm{~cm}, \mathrm{CH}_{2}^{-}\right.$

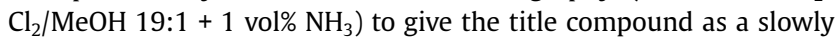
crystallizing yellow oil (43.3 mg, $130 \mu \mathrm{mol}, 78 \%) ; R_{f}=0.54\left(\mathrm{CH}_{2^{-}}\right.$ $\left.\mathrm{Cl}_{2} / \mathrm{MeOH} 9: 1+1 \mathrm{vol} \% \mathrm{NH}_{3}\right)\left[\mathrm{UV}, \mathrm{KMnO}_{4}\right] ;[\alpha]_{\mathrm{D}}{ }^{20}-66\left(c=1.00, \mathrm{CH}_{2^{-}}\right.$ $\mathrm{Cl}_{2}$ ).

IR (ATR): 3360 (w, N-H), 3058 (w, ArH), 2929 (m, CH CH $_{2} 2855$ (w, C-H), $2222(\mathrm{w}, \mathrm{C} \equiv \mathrm{C}), 1639(\mathrm{~m}, \mathrm{C}=\mathrm{O}), 1591(\mathrm{~m}, \mathrm{~N}-\mathrm{H}), 1461(\mathrm{~m}), 1438(\mathrm{~m})$, $1397(\mathrm{~m}), 1326(\mathrm{~m}), 1079(\mathrm{w}), 917(\mathrm{w}), 742(\mathrm{~m}), 635 \mathrm{~cm}^{-1}(\mathrm{w})$.

${ }^{1} \mathrm{H} \mathrm{NMR}\left(400 \mathrm{MHz}, \mathrm{CDCl}_{3}\right): \delta=1.27-1.36(\mathrm{~m}, 1 \mathrm{H}, \mathrm{H}-5), 1.46-1.78(\mathrm{~m}$, $8 \mathrm{H}, \mathrm{H}-3, \mathrm{H}-4, \mathrm{H}-5, \mathrm{H}-6, \mathrm{NH}_{2}$ ), 1.92-2.00 (m, $1 \mathrm{H}, \mathrm{H}-6$ ), 2.83 (virt. dt, ${ }^{3} J=10.2 \mathrm{~Hz},{ }^{3} \cong \cong{ }^{3} \mathrm{~J}=3.9 \mathrm{~Hz}, 1 \mathrm{H}, \mathrm{H}-2$ ), 3.04 (virt. q, ${ }^{3} \mathrm{~J} \cong{ }^{3} \mathrm{~J}=4.1 \mathrm{~Hz}, 1 \mathrm{H}$, H-1), 7.45-7.51 (m, $\left.2 \mathrm{H}, \mathrm{H}-7^{\prime}, \mathrm{H}-4^{\prime}\right), 7.55$ (d, $\left.{ }^{3} \mathrm{~J}=7.7 \mathrm{~Hz}, 1 \mathrm{H}, \mathrm{H}-5^{\prime}\right)$, 7.59-7.64 (m, 2 H, H-6', H-3'), 8.60 (dd, ${ }^{3} J=8.1 \mathrm{~Hz},{ }^{4} \mathrm{~J}=1.5 \mathrm{~Hz}, 1 \mathrm{H}, \mathrm{H}-$ $\left.8^{\prime}\right), 8.64\left(\mathrm{~d},{ }^{4} \mathrm{~J}=1.7 \mathrm{~Hz}, 1 \mathrm{H}, \mathrm{H}-1^{\prime}\right)$.

${ }^{13} \mathrm{C}$ NMR (101 MHz, $\left.\mathrm{CDCl}_{3}\right): \delta=21.8(\mathrm{t}, \mathrm{C}-5), 24.6(\mathrm{t}, \mathrm{C}-4), 30.2(\mathrm{t}, \mathrm{C}-6)$, $32.7(\mathrm{t}, \mathrm{C}-3), 37.5(\mathrm{~d}, \mathrm{C}-1), 51.9(\mathrm{~d}, \mathrm{C}-2), 83.2(\mathrm{~s}, \mathrm{CHC} \equiv \mathrm{C}), 92.0(\mathrm{~s}$, $\mathrm{CHC} \equiv \mathrm{C}), 122.2\left(\mathrm{~s}, \mathrm{C}-2^{\prime}\right), 126.1$ (d, C-5'), $126.2\left(\mathrm{~d}, \mathrm{C}-4^{\prime}\right), 126.6$ (d, C-7'), 129.1 (s, C-8a'), 129.2 (s, C-1a'), 130.1 (d, C-8'), 132.5 (d, C-6'), 133.0 (d, C-1'), 135.0 (d, C-3'), 136.6 (s, C-4a'), 137.0 (s, C-5a'), 179.5 (s, $\mathrm{C}=0$ ).

MS (EI, $70 \mathrm{eV}): m / z(\%)=333\left(100[\mathrm{M}]^{+}\right), 290\left(89,\left[\mathrm{C}_{19} \mathrm{H}_{14} \mathrm{OS}\right]^{+}\right), 237$ $\left(89,\left[\mathrm{C}_{15} \mathrm{H}_{9} \mathrm{OS}\right]^{+}\right), 139\left(11,\left[\mathrm{C}_{7} \mathrm{H}_{7} \mathrm{OS}\right]^{+}\right)$.

HRMS (ESI): $m / z[M+H]^{+}$calcd for $\mathrm{C}_{21} \mathrm{H}_{20} \mathrm{NOS}$ : 334.1260; found: 334.1259. 
1-[3",5"-Bis(trifluoromethyl)phenyl]-3-\{(1S,2S)-2-[(9'-oxo-9'Hthioxanthen-2'-yl)ethynyl]cyclohexyl\}thiourea (4)

To a solution of amine 32 ( $240 \mathrm{mg}, 730 \mu \mathrm{mol}, 1.00$ equiv) in THF (11 $\mathrm{mL})$ was added isothiocyanate $\mathbf{1 3}(145 \mu \mathrm{L}, 214 \mathrm{mg}, 791 \mu \mathrm{mol}, 1.10$ equiv) and the reaction mixture was stirred for $16 \mathrm{~h}$ at $\mathrm{rt}$. Then, the solvent was removed under reduced pressure and the crude product was purified by flash chromatography $(3 \times 15 \mathrm{~cm}$, Chx/EtOAc 9:1 $\rightarrow$ 4:1). The product 4 was isolated as a bright yellow solid ( $330 \mathrm{mg}, 550$ $\mu \mathrm{mol}, 74 \%$ ); $\mathrm{mp} \mathrm{201-202}{ }^{\circ} \mathrm{C} ; R_{f}=0.43$ (Chx/EtOAc 4:1) [UV, $\mathrm{KMnO}_{4}$ ]; $[\alpha]_{\mathrm{D}}{ }^{20}-104\left(c=1.00, \mathrm{CH}_{2} \mathrm{Cl}_{2}\right)$.

IR (ATR): 3327 (br w, N-H), 3061 (w, ArH), 2934 (w, CH CH $_{2} 2858$ (w, CH), 2223 (w, C =C), 1618 (m, C=O), $1585(\mathrm{~m}, \mathrm{~N}-\mathrm{H}), 1523(\mathrm{~m}, \mathrm{~N}-\mathrm{H})$, 1276 (m, C-F), 1172 (s, C=S), 1128 (s), $986(\mathrm{~m}), 884(\mathrm{~m}), 744(\mathrm{~m}), 681$ $\mathrm{cm}^{-1}(\mathrm{~m})$.

${ }^{1} \mathrm{H}$ NMR $\left(500 \mathrm{MHz}, \mathrm{CDCl}_{3}\right): \delta=1.40-1.50(\mathrm{~m}, 1 \mathrm{H}, \mathrm{H}-5), 1.59-1.67(\mathrm{~m}$, $2 \mathrm{H}, \mathrm{H}-4), 1.67-1.73$ (m, $1 \mathrm{H}, \mathrm{H}-3), 1.77$ (td, ${ }^{3} \mathrm{~J}=12.4 \mathrm{~Hz}, 3.8 \mathrm{~Hz}, 1 \mathrm{H}, \mathrm{H}-$ 6), 1.82-1.88 (m, $1 \mathrm{H}, \mathrm{H}-5), 1.95-2.01(\mathrm{~m}, 1 \mathrm{H}, \mathrm{H}-3), 2.06-2.12$ (m, 1 $\mathrm{H}, \mathrm{H}-6$ ), 3.48 (virt. q, ${ }^{3} \mathrm{~J} \cong 3 \mathrm{~J}=4.0 \mathrm{~Hz}, 1 \mathrm{H}, \mathrm{H}-2$ ), 4.65 (virt. ddt, ${ }^{3} \mathrm{~J}=11.8$ $\left.\mathrm{Hz}, 7.7 \mathrm{~Hz},{ }^{3} \mathrm{~J} \cong 3^{3}=3.5 \mathrm{~Hz}, 1 \mathrm{H}, \mathrm{H}-1\right), 7.04\left(\mathrm{~d},{ }^{3} \mathrm{~J}=7.7 \mathrm{~Hz}, 1 \mathrm{H}, \mathrm{H}-3^{\prime}\right)$, $7.12\left(\mathrm{~d},{ }^{3} \mathrm{~J}=8.3 \mathrm{~Hz}, 1 \mathrm{H}, \mathrm{H}-4^{\prime}\right), 7.38\left(\mathrm{~d}, 3^{3}=8.7 \mathrm{~Hz}, 1 \mathrm{H}\right.$, cyclohexylNHCS), 7.49-7.54 (m, 2 H, H-5', H-4"), 7.58 (dd, ${ }^{3} J=8.1 \mathrm{~Hz},{ }^{4} \mathrm{~J}=0.7 \mathrm{~Hz}$, $1 \mathrm{H}, \mathrm{H}-7^{\prime}$ ), 7.66 (ddd, ${ }^{3} \mathrm{~J}=8.3 \mathrm{~Hz}, 7.0 \mathrm{~Hz}, 1.3 \mathrm{~Hz}, 1 \mathrm{H}, \mathrm{H}-6^{\prime}$ ), 7.99 (br s, 2 H, H-2", H-6"), 8.32 (d, J J = 1.6 Hz, $1 \mathrm{H}, \mathrm{H}-1^{\prime}$ ), 8.50 (dd, ${ }^{3} J=8.2 \mathrm{~Hz},{ }^{4} J=$ $\left.1.3 \mathrm{~Hz}, 1 \mathrm{H}, \mathrm{H}-8^{\prime}\right), 9.11$ (s, $\left.1 \mathrm{H}, \mathrm{Ar}-\mathrm{NHCS}\right)$.

${ }^{13} \mathrm{C}$ NMR (101 MHz, $\left.\mathrm{CDCl}_{3}\right): \delta=21.1(\mathrm{t}, \mathrm{C}-4), 25.1(\mathrm{t}, \mathrm{C}-5), 28.6(\mathrm{t}, \mathrm{C}-6)$, $30.3(\mathrm{t}, \mathrm{C}-3), 33.5(\mathrm{~d}, \mathrm{C}-2), 54.9(\mathrm{~d}, \mathrm{C}-1), 83.2(\mathrm{~s}, \mathrm{CHC} \equiv \mathrm{C}), 91.4(\mathrm{~s}$, CHC $\equiv$ C), 118.4 (d, C-4"), 121.7 (s, C-2'), 123.7 (d, C-2"), 123.8 (d, C-6"), $124.5\left(\mathrm{~s}, \mathrm{CF}_{3}\right), 125.7\left(\mathrm{~d}, \mathrm{C}-4^{\prime}\right), 126.3\left(\mathrm{~d}, \mathrm{C}-7^{\prime}\right), 127.0\left(\mathrm{~d}, \mathrm{C}-5^{\prime}\right), 128.3(\mathrm{~s}$, C-1a'), 128.6 (s, C-8a'), 129.8 (d, C-8'), 131.6 (s, C-3"), $132.0\left(\mathrm{~s}, \mathrm{CF}_{3}\right)$, 132.3 (s, C-5"), 132.5 (d, C-1'), 133.1 (d, C-6'), 134.8 (d, C-3'), 137.0 (s, C-4a'), 137.6 (s, C-5a'), 140.4 (s, C-1"), 180.0 (s, C=S), 180.3 (s, C=O).

MS (ESI): $m / z=622\left[\mathrm{M}+\mathrm{NH}_{4}\right]^{+}$.

HRMS (ESI): $m / z[\mathrm{M}+\mathrm{H}]^{+}$calcd for $\mathrm{C}_{30} \mathrm{H}_{23} \mathrm{~F}_{6} \mathrm{~N}_{2} \mathrm{OS}_{2}$ : 605.1151; found: 605.1149 .

\section{8-Bromo-2,2,9b-trimethyl-2,3,4a,9b-tetrahydro-1H-dibenzofu- ran-4-one (34)}

2-(4-Bromophenoxy)-3,5,5-trimethyl-2-cyclohexen-1-one (33; 30.6 $\mathrm{mg}, 100 \mu \mathrm{mol}, 1.00$ equiv) was irradiated together with thiourea 4 (6.04 mg, $10.0 \mu \mathrm{mol}, 0.10$ equiv) in a solution of $\mathrm{CH}_{2} \mathrm{Cl}_{2}(c=20 \mathrm{mM})$ at $\lambda=419 \mathrm{~nm}$ for $24 \mathrm{~h}$ at $\mathrm{rt}$. The reaction was stopped, all volatiles were removed under reduced pressure, and the crude product was purified by flash chromatography $\left(2 \times 15 \mathrm{~cm}, \mathrm{Pn} / \mathrm{Et}_{2} \mathrm{O} 4: 1 \rightarrow 2: 1\right)$. The title compound was isolated as a yellowish oil ( $8.1 \mathrm{mg}, 26.0 \mu \mathrm{mol}, 26 \%, 12 \%$ ee) together with residual starting material ( $15.8 \mathrm{mg}, 51.0 \mu \mathrm{mol}, 54 \%)$; $R_{f}=0.38\left(\mathrm{Pn} / \mathrm{Et}_{2} \mathrm{O} 2: 1\right)[\mathrm{CAM}] ;[\alpha]_{\mathrm{D}}{ }^{20}+10\left(c=1.00, \mathrm{CH}_{2} \mathrm{Cl}_{2}\right)$.

Chiral HPLC (AD-H, $250 \times 4.6 \mathrm{~mm}, n$-heptane/i-PrOH (90:10), 1 $\mathrm{mL} / \mathrm{min}, \lambda=210 \mathrm{~nm}, 254 \mathrm{~nm}): t_{\mathrm{R}}$ (racemate) $=10.3 \mathrm{~min}(34), 13.2 \mathrm{~min}$ (ent-34).

${ }^{1} \mathrm{H}$ NMR $\left(400 \mathrm{MHz}, \mathrm{CDCl}_{3}\right): \delta=0.61\left[\mathrm{~s}, 3 \mathrm{H}, \mathrm{C}-2\left(\mathrm{CH}_{3}\right)\right], 1.11[\mathrm{~s}, 3 \mathrm{H}, \mathrm{C}-$ 2( $\left.\left(\mathrm{CH}_{3}\right)\right], 1.40\left[\mathrm{~s}, 3 \mathrm{H}, \mathrm{C}-9 \mathrm{~b}\left(\mathrm{CH}_{3}\right)\right], 1.95\left(\mathrm{~d},{ }^{2} \mathrm{~J}=14.8 \mathrm{~Hz}, 1 \mathrm{H}, \mathrm{CHH}-1\right)$, 2.19-2.24 (m, $2 \mathrm{H}, \mathrm{CHH}-1, \mathrm{CHH}-3$ ), 2.37 (d, ${ }^{2} \mathrm{~J}=12.7 \mathrm{~Hz}, 1 \mathrm{H}, \mathrm{CHH}-3$ ), 4.54 (s, $1 \mathrm{H}, \mathrm{H}-4 \mathrm{a}), 6.83\left(\mathrm{~d},{ }^{3} \mathrm{~J}=8.5 \mathrm{~Hz}, 1 \mathrm{H}, \mathrm{H}_{\mathrm{Ar}}\right), 7.13\left(\mathrm{~d},{ }^{4} \mathrm{~J}=2.1 \mathrm{~Hz}, 1\right.$ $\left.\mathrm{H}, \mathrm{H}_{\mathrm{Ar}}\right), 7.24\left(\mathrm{dd},{ }^{3} \mathrm{~J}=8.5 \mathrm{~Hz},{ }^{4} \mathrm{~J}=2.1 \mathrm{~Hz}, 1 \mathrm{H}, \mathrm{H}_{\mathrm{Ar}}\right)$.

${ }^{13} \mathrm{C}$ NMR $\left(101 \mathrm{MHz}, \mathrm{CDCl}_{3}\right): \delta=27.1$ [q, C-2 $\left.\left(\mathrm{CH}_{3}\right)\right], 32.4$ [q, C-2 $\left(\mathrm{CH}_{3}\right)$ ], 32.4 [q, C-9b $\left(\mathrm{CH}_{3}\right)$ ], 36.1 (s, C-2), 46.0 (t, C-1), 49.6 (s, C-9b), 51.8 (t, C3), 91.1 (d, C-4a), 112.4 (d, Car), 113.5 (s, Car), 125.1 (d, Car), 131.4 (d, Car), 136.9 (s, Car), 157.0 (s, Car), 207.2 (s, C-4).

The spectroscopic data match the literature values. ${ }^{3 \mathrm{~h}}$

\section{Funding Information}

Financial support by the European Research Council under the European Union's Horizon 2020 research and innovation programme (grant agreement No 665951 - ELICOS) and the Alexander von Humboldt-Stiftung (postdoctoral fellowship to E. R.) is gratefully acknowledged.

\section{Supporting Information}

Supporting information for this article is available online at https://doi.org/10.1055/s-0036-1590931.

\section{Primary Data}

for this article are available online at https://doi.org/10.1055/s-00361590931 and can be cited using the following DOI: 10.4125/pd0096th.

\section{References}

(1) Meier, K.; Zweifel, H. J. Photochem. 1986, 35, 353.

(2) (a) Amirzadeh, G.; Schabel, W. Makromol. Chem. 1981, 182, 2821. (b) Allonas, X.; Ley, C.; Bibaut, C.; Jacques, P.; Fouassier, J. P. Chem. Phys. Lett. 2000, 322, 483.

(3) Examples: (a) Hosaka, S.; Wakamatsu, S. Tetrahedron Lett. 1968, 219. (b) Padwa, A.; Blacklock, T. J. J. Am. Chem. Soc. 1977, 99, 2345. (c) Schultz, P. G.; Dervan, P. B. J. Am. Chem. Soc. 1982, 104, 6660. (d) Padwa, A.; Kennedy, G. D.; Wannamaker, M. W. J. Org. Chem. 1985, 50, 5334. (e) Zimmerman, H. E.; Caufield, C. E.; King, R. K. J. Am. Chem. Soc. 1985, 107, 7732. (f) Armesto, D.; Ortiz, M. J.; Agarrabeitia, A. R.; El-Boulifi, N. Angew. Chem. Int. Ed. 2005, 44, 7739. (g) Mayr, F.; Brimioulle, R.; Bach, T. J. Org. Chem. 2016, 81, 6965. (h) Edtmüller, V.; Bach, T. Tetrahedron 2017, 33, 5038 .

(4) Review: Brimioulle, R.; Lenhart, D.; Maturi, M. M.; Bach, T. Angew. Chem. Int. Ed. 2015, 54, 3872.

(5) (a) Alonso, R.; Bach, T. Angew. Chem. Int. Ed. 2014, 53, 4368. (b) Tröster, A.; Alonso, R.; Bach, T. J. Am. Chem. Soc. 2016, 138, 7808.

(6) For a DFT study on the mechanism of this transformation, see: Yang, Y.; Wen, Y.; Dang, Z.; Yu, H. J. Phys. Chem. A 2017, 121, 4552.

(7) Ding, W.; Lu, L.-Q.; Zhou, Q.-Q.; Wei, Y.; Chen, J.-R.; Xiao, W.-J. J. Am. Chem. Soc. 2017, 139, 63.

(8) Reviews: (a) Hof, K.; Lippert, K. M.; Schreiner, P. R. In Science of Synthesis: Asymmetric Organocatalysis; List, B.; Maruoka, K., Eds.; Thieme: Stuttgart, 2012, 297-412. (b) Kotke, M.; Schreiner, P. R. In Hydrogen Bonding in Organic Synthesis; Pihko, P. M., Ed.; Wiley-VCH: Weinheim, 2009, 141-251. (c) Connon, S. J. Chem. Eur. J. 2006, 12, 5418. (d) Taylor, M. S.; Jacobsen, E. N. Angew. Chem. Int. Ed. 2006, 45, 1520.

(9) For recent work, see: (a) Robertson, G. P.; Farley, A. J. M.; Dixon, D. J. Synlett 2016, 27, 21. (b) Jovanovic, P.; Petkovic, M.; Ivkovic, B.; Savic, V. Tetrahedron: Asymmetry 2016, 27, 990. (c) Zhao, K.; Zhi, Y.; Shu, T.; Valkonen, A.; Rissanen, K.; Enders, D. Angew. Chem. Int. Ed. 2016, 55, 12104. (d) Yan, L.-J.; Wang, H.-F.; Chen, W.-X.; Tao, Y.; Jin, K.-J.; Chen, F.-E. ChemCatChem 2016, 8, 2249. (e) Günler, Z. I.; Alfonso, I.; Jimeno, C.; Pericàs, M. A. Synthesis 2017, 49, 319. (f) Otevrel, J.; Bobal, P. Synthesis 2017, 49, 593. 
(g) Jarvis, C. L.; Hirschi, J. S.; Vetticatt, M. J.; Seidel, D. Angew. Chem. Int. Ed. 2017, 56, 2670. (h) Meninno, S.; Overgaard, J.; Lattanzi, A. Synthesis 2017, 49, 1509.

(10) (a) Vallavoju, N.; Selvakumar, S.; Jockusch, S.; Sibi, M. P.; Sivaguru, J. Angew. Chem. Int. Ed. 2014, 53, 5604. (b) Vallavoju, N.; Selvakumar, S.; Jockusch, S.; Prabhakaran, M. T.; Sibi, M. P.; Sivaguru, J. Adv. Synth. Catal. 2014, 356, 2763.

(11) For a Lewis acid catalyzed version of the reaction, see: (a) Guo, H.; Herdtweck, E.; Bach, T. Angew. Chem. Int. Ed. 2010, 49, 7782. (b) Brimioulle, R.; Guo, H.; Bach, T. Chem. Eur.J. 2012, 18, 7552.

(12) Vallavoju, N.; Selvakumar, S.; Pemberton, B. C.; Jockusch, S.; Sibi, M. P.; Sivaguru, J. Angew. Chem. Int. Ed. 2016, 55, 5446.

(13) Telmesani, R.; Park, S. H.; Lynch-Colameta, T.; Beeler, A. B. Angew. Chem. Int. Ed. 2015, 54, 11521.

(14) Lippert, K. M.; Hof, K.; Gerbig, D.; Ley, D.; Hausmann, H.; Guenther, S.; Schreiner, P. R. Eur. J. Org. Chem. 2012, 5919.

(15) (a) Bertucci, M. A.; Lee, S. J.; Gagné, M. R. Chem. Commun. 2013, 49, 2055. (b) Tripathi, C. B.; Mukherjee, S. J. Org. Chem. 2012, 77, 1592. (c) Zhang, X.-J.; Liu, S.-P.; Lao, J.-H.; Du, G.-J.; Yan, M.; Chan, A. S. C. Tetrahedron: Asymmetry 2009, 20, 1451. (d) Alemán, J.; Milelli, A.; Cabrera, S.; Reyes, E.; Jørgensen, K. A. Chem. Eur. J. 2008, 14, 10958.

(16) (a) Stack, J. G.; Curran, D. P.; Geib, S. V.; Rebek, J. Jr.; Ballester, P. J. Am. Chem. Soc. 1992, 114, 7007. (b) Bach, T.; Bergmann, H.; Harms, K. Angew. Chem. Int. Ed. 2000, 39, 2302. (c) Bach, T.; Bergmann, H.; Grosch, B.; Harms, K.; Herdtweck, E. Synthesis 2001, 1395. (d) Bauer, A.; Westkämper, F.; Grimme, S.; Bach, T. Nature 2005, 436, 1139. (e) Müller, C.; Bauer, A.; Bach, T. Angew. Chem. Int. Ed. 2009, 48, 6640.

(17) (a) Jares-Erijman, E. A.; Bapat, C. P.; Lithgow-Bertelloni, A.; Rinehart, K. L.; Sakai, R.J. Org. Chem. 1993, 58, 5732. (b) Vincent, A.; Deschamps, D.; Martzel, T.; Lohier, J.-F.; Richards, C. J.; Gaumont, A.-C.; Perrio, S. J. Org. Chem. 2016, 81, 3961.

(18) Xing, R.-G.; Li, Y.-N.; Liu, Q.; Meng, Q.-Y.; Li, J.; Shen, X.-X.; Liu, Z.; Zhou, B.; Yao, X.; Liu, Z.-L. Eur. J. Org. Chem. 2010, 6627.

(19) Lee, H.; Kim, M.; Jun, Y. M.; Kim, B. H.; Lee, B. M. Heteroat. Chem. 2011, 22, 158.

(20) (a) Hou, J.; Li, Z.; Fang, Q.; Feng, C.; Zhang, H.; Guo, W.; Wang, H.; Gu, G.; Tian, Y.; Liu, P.; Liu, R.; Lin, J.; Shi, Y.-K.; Yin, Z.; Shen, J.; Wang, P. G. J. Med. Chem. 2012, 55, 3066. (b) Belema, M.; Nguyen, V. N.; Romine, J. L.; St. Laurent, D. R.; Lopez, O. D.; Goodrich, J. T.; Nower, P. T.; O’Boylell, D. R.; Lemm, J. A.; Fridell, R. A.; Gao, M.; Fang, H.; Krause, R. G.; Wang, Y.-K.; Oliver, A. J.; Good, A. C.; Knipe, J. O.; Meanwell, N. A.; Snyder, L. B. J. Med. Chem. 2014, 57, 1995.

(21) (a) Dondas, H. A.; Grigg, R.; Kilner, C. Tetrahedron 2003, 59, 8481. (b) Contreras, J.-M.; Rival, Y. M.; Chayer, S.; Bourguignon, J.-J.; Wermuth, C. G. J. Med Chem. 1999, 42, 730.

(22) (a) Forró, E.; Fülöp, F. Tetrahedron: Asymmetry 2004, 15, 573. (b) Chisholm, C. D.; Fülöp, F.; Forró, E.; Wenzel, T. J. Tetrahedron: Asymmetry 2010, 21, 2289.
(23) (a) Bolm, C.; Gerlach, A.; Dinter, C. L. Synlett 1999, 195. (b) Bolm, C.; Schiffers, I.; Dinter, C. L.; Gerlach, A. J. Org. Chem. 2000, 65, 6984.

(24) (a) Arora, I.; Sha, A. K. Tetrahedron 2016, 72, 5479. (b) Cao, X.-Y.; Zheng, J.-C.; Li, Y.-X.; Shu, Z.-C.; Sun, X.-L.; Wang, B.-Q.; Tang, Y. Tetrahedron 2010, 66, 9703.

(25) (a) Bandyopadhyay, D.; Cruz, J.; Banik, B. K. Tetrahedron 2012, 68, 10686. (b) Chen, K.-T.; Huang, D.-Y.; Chiu, C.-H.; Lin, W.-W.; Liang, P.-H.; Cheng, W.-C. Chem. Eur. J. 2015, 21, 11984.

(26) Benzoyl deprotection: Shreykar, M. R.; Sekar, N. Tetrahedron Lett. 2016, 57, 4174.

(27) For a previous synthesis of 21, see: Viña, D.; Santana, L.; Uriarte, E.; Quezada, E.; Valencia, L. Synthesis 2004, 2517.

(28) Reich H. J.; Structure Determination by NMR; Vicinal ProtonProton Coupling ${ }^{3} J_{H H}$; Chap. 5.05; retrieved from https://www.chem.wisc.edu/areas/reich/chem605/ (accessed Aug. 1, 2017)

(29) The A-value for the parent ethynyl group is tabulated as 0.410.52: Eliel, E. L.; Wilen, S. H. Stereochemistry of Organic Compounds; Wiley-Interscience: New York, 1994, 696-697.

(30) (a) Inokuma, T.; Suzuki, Y.; Sakaeda, T.; Takemoto, Y. Chem. Asian J. 2011, 6, 2902. (b) Neubert, A.; Barnes, D.; Kwak, Y.-S.; Nakajima, K.; Bebernitz, G. R.; Coppola, G. M.; Kirman, L.; Serrano-Wu, M. H.; Stams, T.; Topiol, S. W.; Vedananda, T. F.; Wareing, J. R. Patent WO2007115058 (A2), 2007.

(31) (a) Mancuso, A. J.; Huang, S-L.; Swern, D. J. Org. Chem. 1978, 43, 2480. (b) De Lucca, G. V.; Kim, U. T.; Vargo, B. J.; Duncia, J. V.; Santella, J. B. III.; Gardner, D. S.; Zheng, C.; Liauw, A.; Wang, Z.; Emmett, G.; Wacker, D. A.; Welch, P. K.; Covington, M.; Stowell, N. C.; Wadman, E. A.; Das, A. M.; Davies, P.; Yeleswaram, S.; Graden, D. M.; Solomon, K. A.; Newton, R. C.; Trainor, G. L.; Decicco, C. P.; Ko, S. S. J. Med. Chem. 2005, 48, 2194.

(32) (a) Seyferth, D.; Marmor, R. S.; Hilbert, P. J. Org. Chem. 1971, 36, 1379. (b) Gilbert, J. C.; Weerasooriya, U. J. Org. Chem. 1979, 44, 4997. (c) Hauske, J. R.; Dorff, P.; Julin, S.; Martinelli, G.; Bussolari, J. Tetrahedron Lett. 1992, 33, 3715.

(33) (a) Sonogashira, K.; Tohda, Y.; Hagihara, N. Tetrahedron Lett. 1975, 4467. (b) Krishnendu, B.; Soumen, S.; Swapnadeep, J.; Umasish, J. J. Org. Chem. 2012, 77, 8780.

(34) Gilman, H.; Diehl, J. W. J. Org. Chem. 1959, 24, 1914.

(35) (a) Schultz, A. G.; Lucci, R. D. J. Org. Chem. 1975, 40, 1371. (b) Schultz, A. G.; Lucci, R. D.; Fu, W. Y.; Berger, M. H.; Erhardt, J.; Hagmann, W. K. J. Am. Chem. Soc. 1978, 100, 2150. (c) Burke, T. R. Jr.; Jacobson, A. E.; Rice, K. C.; Silverton, J. V. J. Org. Chem. 1984, 49, 1051.

(36) Münster, N.; Parker, N. A.; van Dijk, L.; Paton, R. S.; Smith, M. D. Angew. Chem. Int. Ed. 2017, 56, 9468.

(37) Armarego, W. L. F.; Chai, C. L. L. Purification of Laboratory Chemicals; Butterworth-Heinemann: Burlington USA, 2003, 29-30. 